\title{
The effects of a randomized four-week Graston Instrumented- Assisted Soft Tissue Mobilization (GISTM) dynamic balancing- training program on individuals with chronic ankle instability
}

\author{
Jessica L. Schaefer
}

West Virginia University

Follow this and additional works at: https://researchrepository.wvu.edu/etd

\section{Recommended Citation \\ Schaefer, Jessica L., "The effects of a randomized four-week Graston Instrumented-Assisted Soft Tissue Mobilization (GISTM) dynamic balancing-training program on individuals with chronic ankle instability" (2009). Graduate Theses, Dissertations, and Problem Reports. 2781.}

https://researchrepository.wvu.edu/etd/2781

This Thesis is protected by copyright and/or related rights. It has been brought to you by the The Research Repository @ WVU with permission from the rights-holder(s). You are free to use this Thesis in any way that is permitted by the copyright and related rights legislation that applies to your use. For other uses you must obtain permission from the rights-holder(s) directly, unless additional rights are indicated by a Creative Commons license in the record and/ or on the work itself. This Thesis has been accepted for inclusion in WVU Graduate Theses, Dissertations, and Problem Reports collection by an authorized administrator of The Research Repository @ WVU. For more information, please contact researchrepository@mail.wvu.edu. 
The Effects of a Randomized Four-Week Graston Instrumented-Assisted Soft Tissue Mobilization (GISTM) Dynamic Balancing-Training Program on Individuals with Chronic Ankle Instability

Jessica L. Schaefer, BS, ATC

Thesis submitted to the College of Physical Activity and Sport Sciences at West Virginia University in partial fulfillment of the requirements for the degree of

Master of Science in Athletic Training

Michelle A. Sandrey, PhD, ATC, Chair

Sam Mukdadi, PhD

Vicki Williams, MD

College of Physical Activity and Sport Sciences

Morgantown, West Virginia

2009

Keywords: Chronic Ankle Instability, Graston Instrumented-Assisted Soft Tissue Mobilization, Dynamic Balance-Training, Star Excursion Balance Test, Ultrasonography, Foot and Ankle Ability Measures and FAAM Sport, Visual Analog Scale 


\begin{abstract}
The Effects of a Randomized Four-Week Graston Instrumented-Assisted Soft Tissue Mobilization Dynamic Balancing-Training Program on Individuals with Chronic Ankle Instability
\end{abstract}

Jessica L. Schaefer

Context: Despite case reports and clinical studies, there have been no experimental studies conducted on Graston Instrumented-Assisted Soft Tissue Mobilization (GISTM) technique and the effects on chronic ankle instability. Most of the clinical studies and case reports that have been conducted have used animals and human subjects for lateral epicondylitis, and patellar, rotator cuff and achilles tendinopathy. Since only a case report exists in the literature for chronic ankle instability using GISTM, the intent of this study is to further evaluate the effects of GISTM and dynamic postural balance on improvements in healing and function. Objective: The present study aims to investigate the affects of GISTM technique and the influence on improving range of motion, neovascularization, collagen alignment, pain and disability in individuals experiencing chronic ankle instability. Design: Subjects were randomly assigned to the GISTM/ Dynamic Balance-Training (DBT), GISTM-S/DBT, or C/DBT group. All groups participated in a 4wk DBT program consisting of four exercises and a lower extremity dynamic stretching protocol using a Flex band twice a week throughout the training period. The GISTM/DBT and GISTM-S/DBT groups received the GISTM treatment of sham treatment twice a week before performing the DBT program throughout the training period. Setting: A AAA High School in North Central West Virginia. Patients or Other Participants: This study included 36 healthy, physically active individuals ( 5 female, 31 male; age $=17.771 \pm 1.972$ years; $h t=69.403 \pm 5.775 ; 30$ right, 6 left foot; 28 six sessions, 8 seven sessions completed of rehab) with a history of CAI volunteered to be in this study from a North Central High School in WV and a DI Mid-Atlantic University. Interventions: The DBT program that was performed twice a week for 4-wks included a battery of exercises that was advanced from week to week for all three experimental groups. The 4-wk GISTM treatment protocol for the GISTM and GISTM-S groups also was performed twice a week. Pre- and Post-test measurements were taken using the Foot and Ankle Ability Measures (FAAM), FAAM Sport, the Visual Analog Scale (VAS), ankle range of motion (ROM) in four directions, ultrasonography (US), and the Star Excursion Balance Test (SEBT) in three directions. Outcomes Measures: A greater increase will be found in the talocrural range of motion, neovascularization, and collagen alignment between groups preand post-test. And also a decrease in the FAAM, FAAM Sport and VAS will be found; with a significant difference in pre- and post-test results between groups. Results: There was a significant difference for test with FAAM $\left(\mathrm{F}_{1,33}=47.963, \mathrm{P}=0.01, \mathrm{ES}=0.592,1-\beta=1.00\right)$, FAAM Sport $\left(\mathrm{F}_{1,33}=18.377, \mathrm{P}<0.001, \mathrm{ES}=0.358,1-\beta=0.932\right)$, VAS $\left(\mathrm{F}_{1,33}=55.564, \mathrm{P}<0.001\right.$, $\mathrm{ES}=0.590,1-\beta=1.000), \operatorname{ROM}\left(\mathrm{F}_{1,33}=40.087, \mathrm{P}<0.001, \mathrm{ES}=0.548,1-\beta=1.000\right)$, and SEBT $\left(\mathrm{F}_{1,33}=58.493, \mathrm{P}=0.000, \mathrm{ES}=0.639,1-\beta=1.000\right)$ with post-test results greater except for VAS, which decreased. There were also significant differences for movement $\left(\mathrm{F}_{3,33}=196.721\right.$, $\mathrm{P}<0.001, \mathrm{ES}=0.856,1-\beta=1.000)$ with $\mathrm{ROM}$ and direction $\left(\mathrm{F}_{1,33}=71.355, \mathrm{P}<0.001, \mathrm{ES}=0.684\right.$, $1-\beta=1.000)$ for the SEBT. All directions and movements were significant $(\mathrm{p}>0.01)$. Conclusion: The 4-wk GISTM and dynamic balance-training program could be used to increase ROM, sensorimotor facilitation, and stability for the ankle musculature, along with postural stability and endurance in all athletes suffering from chronic ankle instability. 


\section{ACKNOWLEDGEMENTS}

I would like to extend a huge thanks to my family: Dad, Mom, Jerod and Cody. You have always been there to encourage me to always do my best, and letting me know that I could do whatever I put my mind to. You don't know how much I appreciate you being there for me the 24 years of my life. I will always do my best to make you proud of me.

I would like to thank my mentors, Kip Smith and Robert Black for the past six years of guidance. You both told me to believe in myself and all that I am. And to know that there is something inside me that is greater than any obstacle. I owe you my deepest gratitude for everything that you have taught me personally and professionally. Thank you for believing in me.

I would like to thank the University High School athletes, parents, coaches, and administration for the great experience and acceptance into the high school. You have helped play a part in my next step into the real world.

Dr. Sandrey, thank you for always being there to help me out. You always tried to keep me positive and confident in all that I did when I was struggling with different aspects in Graduate School. All the research studies that you required me to read has helped me out with current thoughts and ideas to incorporate into my daily routine. It is for that reason that I thank you for making me a better researcher and athletic trainer.

I would like to thank Dr. Vicky Williams and Dr. Sam Mukdadi for serving as thesis committee members. Your thoughts and help have made this paper more fluent and understandable. I would like to send a special thanks to Dr. Vicky Williams for her patience, time, and expertise in teaching me how to use and read ultrasound images. I would not have been able to complete my thesis without your assistance.

I would like to thank GRASTON® Technique in providing on loan the use of the GRASTON® Technique Instruments. Your generosity is greatly appreciated and will not be forgotten.

I would like to thank Daniel Tyskiewicz for his time and General Electric in providing the use of the LOGICQ e ultrasound unit. Daniel, thank you so much for taking time out of your busy schedule to bring the unit on three separate occasions. Your time and GE's generosity is greatly appreciated.

I would like to extend a huge thanks to the University High School athletes. Thank you for volunteering to be in the study and being there for the rehabilitation sessions. I enjoyed working with you and seeing you improve from the start to the finish. Good Luck throughout your 2009-10 seasons or your future endeavors. Thanks again for everything. 


\section{TABLE OF CONTENTS}

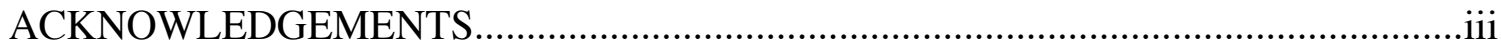

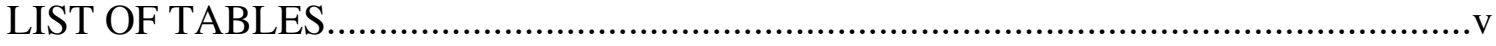

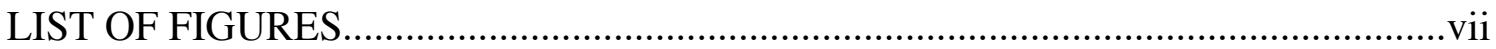

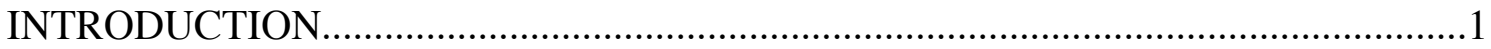

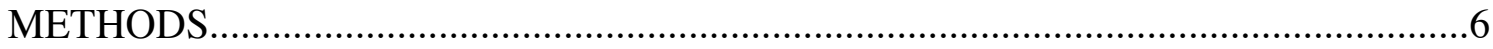

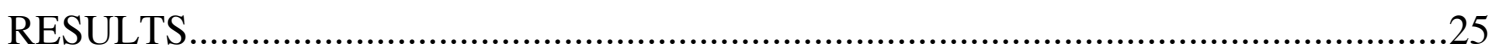

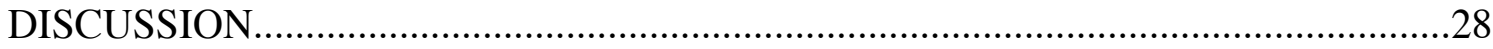

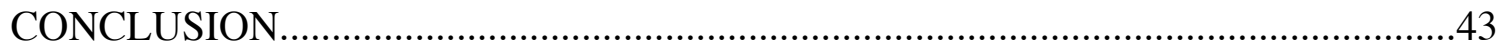

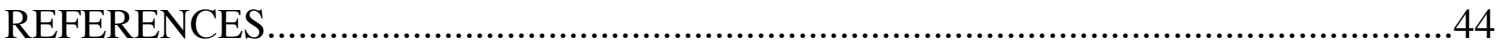

APPENDICES

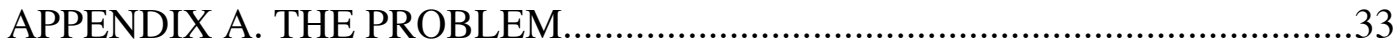

APPENDIX B. LITERATURE REVIEW ..........................................................43

APPENDIX C. ADDITIONAL METHODS................................................... 85

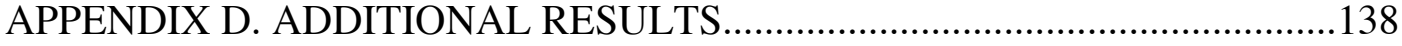

APPENDIX E. RECOMMENDATIONS FOR FUTURE RESEARCH...............149

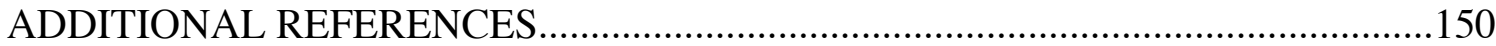




\section{LIST OF TABLES}

B1. Anatomical Location of Talocrural Joint Ligaments............................................64

B2. The Talocrural Joint Muscles................................................................65

B3. Subtalar Ligaments of the Ankle.....................................................................67

B4. Ankle Complex Muscles of the Subtalar Joint.................................68

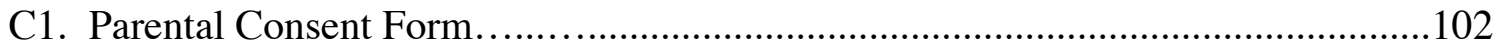

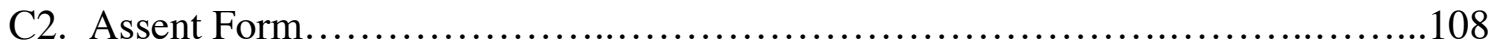

C3. Consent Form...................................................... 113

C4. Authorization to Use or Disclose Protected Health Information (HIPAA)............118

C5. Subject Demographics Questionnaire...........................................................120

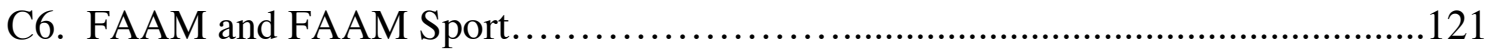

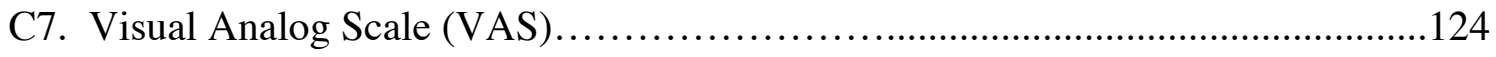

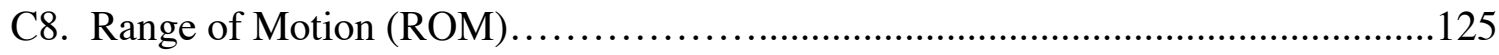

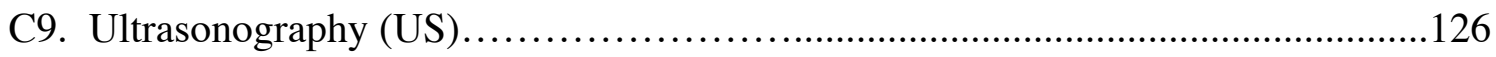

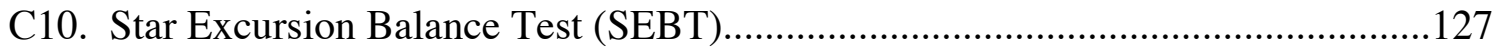

C11. Dynamic Flex Band Flexibility Warm-up Routine..........................................129

C12. Dynamic Balancing-Training Program.................................133

C13. Graston Instrumented-Assisted Soft Tissue Mobilization (GISMT)...................136

D1. Descriptive Statistics on Means and Standard Deviations for all Dependent Variables.............................................................. 138

D2. Two-way ANOVA Repeated Measures Results for FAAM, FAAM Sport, and

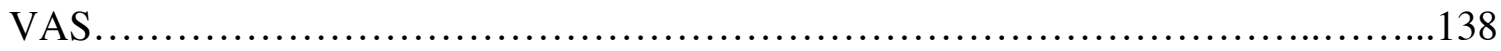

D3. Paired Samples t-Test for Pre-Test and Post-Test for FAAM, FAAM Sport, and

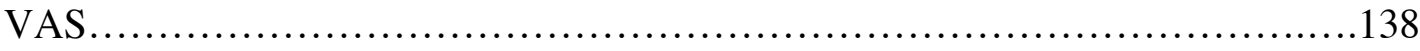


D4. Percent Change Scores for FAAM, FAAM Sport, and VAS....................139

D5. Repeated Measures ANOVA Results for ROM...............................139

D6. Paired Samples t-Test Results for Pre-Test and Post-Test for ROM (Mean

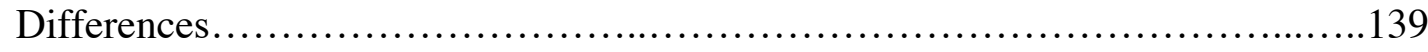

D7. Pairwise Comparison for ROM (Mean Differences)............................. 139

D8. Percent Change Scores for ROM......................................139

D9. Repeated Measures ANOVA Results for SEBT ...............................140

D10. Paired Samples t-Test Results for Pre-Test and Post-Test for SEBT (Mean

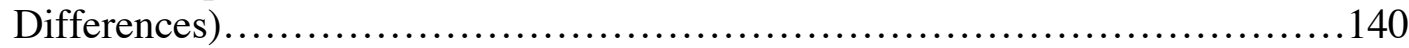

D11. Pairwise Comparison for SEBT (Mean Differences) .........................140

D12. Percent Change Scores for SEBT .......................................140 


\section{LIST OF FIGURES}

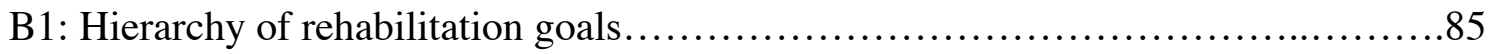

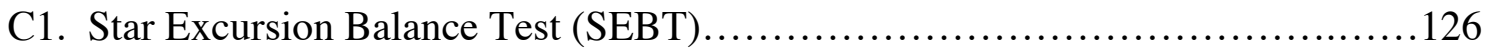

C2. Dynamic Flex Band Flexibility Ankle Warm-up..............................129

C3. Dynamic Flex Band Flexibility Calf Warm-up..................................129

C4. Dynamic Flex Band Flexibility Achilles Warm-up..............................130

C5. Dynamic Flex Band Flexibility Hamstring Warm-up............................130

C6. Dynamic Flex Band Flexibility Groin Warm-up...............................131

C7. Dynamic Flex Band Flexibility IT-Band Warm-up..............................131

C8. Dynamic Flex Band Flexibility Quadriceps/Hip Flexor Warm-up...................132

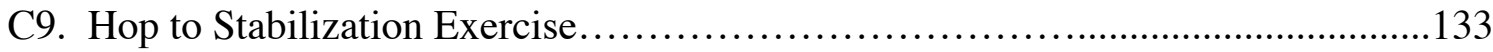

C10. Hop to Stabilization Exercise.........................................................133

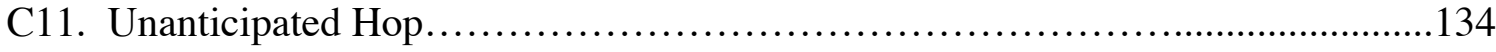

D1. Descriptive Statistics of Pre-Test and Post-Test for FAAM........................141

D2. Descriptive Statistics of Pre-Test and Post-Test for FAAM Sport...................141

D3. Descriptive Statistics of Pre-Test and Post-Test for VAS........................142

D4. Descriptive Statistics of Pre-Test and Post-Test for ROM..........................142

D5. Descriptive Statistics of Pre-Test and Post-Test for SEBT..........................143

D6. Ultrasonography of Normal ATF Ligament....................................143

D7. Ultrasonography of Normal CF Ligament.....................................144

D8. GISTM Group Pre-Test Ultrasonography of ATF Ligament.......................144

D9. GISTM Group Post-Test Ultrasonography of ATF Ligament.......................145

D10. GISTM Group Pre-Test Ultrasonography of CF Ligament........................145 
D11. GISTM Group Post-Test Ultrasonography of CF Ligament...................146

D12. GISTM-Sham Group Pre-Test Ultrasonography of ATF Ligament...............146

D13. GISTM-Sham Group Post-Test Ultrasonography of ATF Ligament..............147

D14. GISTM-Sham Group Pre-Test Ultrasonography of CF Ligament................147

D15. GISTM-Sham Group Post-Test Ultrasonography of CF Ligament.................148 


\section{INTRODUCTION}

The most common sports-related injury is an ankle sprain, more specifically lateral ankle sprains. ${ }^{1,2,3}$ Studies have shown that approximately 10 percent to 25 percent $^{4,5,6,7,8}$ of all sports injuries are ankle sprains. Ankle sprains account for 75 percent of all ankle injuries with 85 percent due to inversion trauma. ${ }^{6,9,10,11}$ Due to the inversion mechanism of injury, predominantly 80 percent to 95 percent of the time, the lateral ligaments of the ankle also affected. ${ }^{9,12,14}$ An estimated 23,00 to 27,000 $0^{1,2,3,4}$ ankle sprains occur per day in the United States, which equals approximately one ankle sprain per $10,000^{1,2,3,4}$ people daily.

The reoccurrence rate of ankle sprains varies in the literature from 20 percent to as high as 80 percent. ${ }^{3,6,7,13,15,16,17,18,19,20}$ After suffering one ankle sprain, an individual is predisposed to chronic ankle instability. Twenty to 40 percent $^{6,7}$ of these patients will go on to experience chronic instability and subsequent disability. However, there is conflicting statistics on the percentage of ankle sprains and those caused by inversion trauma.

Seven percent to 10 percent of the ankle sprains account for emergency room visits. ${ }^{4,5}$ However, an estimated 55 percent of individuals suffering an ankle sprain never seek medical treatment. ${ }^{1}$ Ankle sprains result in more time loss than any other single injury in athletics. Time loss due to ankle injuries is estimated to account for one sixth of all time lost from sport. Safran et al. ${ }^{7}$ stated that approximately 25 percent of time loss in football, basketball, and cross country is due to ankle injuries.

Subsequent injury and time loss due to ankle sprains may be preventable with better understanding of the function of the ligament structures and biomechanics of the 
ankle..$^{4,5,6,7,9,10,13,14,15,16,21}$ In chronic ligament injury, the normal healing process of connective tissue is disrupted. Normal tissue remodeling is prevented by stresses and hypovascularity. ${ }^{24,25,26,27}$ Connective tissue tends to become shorter and denser as it heals, unless beneficial conditions exist. ${ }^{24,25,26,27}$ This contracture and thickening partially results from the increased interfiber bonding in a shortened state. ${ }^{24,25,26,27}$ The individual fibers lose gliding capacity and mobility relative to each other, as do bundles of fibers, and whole ligaments relative to their surrounding structures. ${ }^{24,25,26,27}$ When a lateral ankle sprain occurs, structural damage not only occurs to the ligamentous tissue, but also to the nervous and musculotendinous tissue around the ankle complex ${ }^{10,18,19,29,31}$ The lateral ankle ligaments and joint capsule of the talocrural and subtalar joints have been shown to be extensively innervated by mechanoreceptors. A disruption of the sensory receptors within the lateral ligamentous structures is believed to result in a decreased ability to sense changes in joint position. ${ }^{10,18,19,29,30,31,32}$ While injury to the ligaments may result in laxity of the joints of the ankle complex, neuromuscular deficits are also likely to occur due to the injury to the nervous and musculotendinous tissue. ${ }^{10,18,19,29,30,31}$ These neuromuscular deficits may be manifested as impaired balance, reduced joint position sense, slower firing of the peroneal muscles to inversion perturbation of the ankle, slowed nerve conduction velocity, impaired cutaneous sensation, strength deficits and decreased dorsiflexion range of motion. ${ }^{10,18,19,29,30,31}$

Several studies have been condcuted to evaluate the effectiveness of postural stability programs using chronic ankle instability subjects. ${ }^{33,34,35}$ When compared to a control group, semi-dynamic and dynamic postural stability improved. McKeon et al. ${ }^{35}$ developed a four-week progressive dynamic balance-training program in those with 
chronic ankle instability. He incorporated single-limb hops to stabilization, hop to stabilization and reach, unanticipated hop to stabilization, and single-limb stance activities into a rehabilitation program. All the exercises were designed to challenge the subjects' ability to maintain a single-limb stance while performing various balance activities. ${ }^{35}$ More specifically, the program challenged recovery of single-limb balance efficiently after a disturbance and effectively developed natural execution to accomplish movement goals. ${ }^{35}$ These innovative activities promoted the restoration of functional variability within the sensorimotor system with environmental constraints. ${ }^{35}$ However, although functional ankle instability has been addressed, ${ }^{34,35}$ few studies have addressed the sequlae of scar tissue formation and the healing process with chronic ankle instability.

It is postulated that the healing process in chronic injuries is in accordance with that of acute injuries. When a ligamentous soft tissue injury occurs, one of the damaging mechanisms is scar tissue formation. To assist in removal of excessive scar tissue and the healing process, a type of soft tissue mobilization may be used. Augmented Soft Tissue Mobilization (ASTM) introduces a more controlled amount of microtrauma into an area of excessive scar tissue, therefore, the response of the tendon to this microtrauma could involve augmentation of the healing process. ${ }^{36,37,38,39,40,41}$ Graston Instrumented-Assisted Soft Tissue Mobilization (GISTM), another variation of this technique, also has shown to increase the fibroblast response with the controlled movement of the instruments. ${ }^{2,5,6,7,9,37,44}$ Although most of the original research has focused on tendons using animal and human subjects, there have only been two studies reported using $\mathrm{ASTM}^{41}$ and $\mathrm{GISTM}^{45}$ on ligaments. 
Despite the lack of presence of inflammatory cells, healing is evident following GISTM treatment using an animal model. ${ }^{36,37,38,39,40,41}$ In one study, a significant increase in fibroblast number was noted in two of the experimental groups, which illustrates one of the reported features of tendon healing; proliferation of fibroblasts. ${ }^{36}$ Gait analysis studies, reported evident healing and earlier recovery of limb function following a collagenase injury to the achilles tendon. ${ }^{6,15,16,46,47,48}$ Early restoration of function, promotes mechanical forces to act more rapidly to further collagen fiber realignment and thus accelerate the completion of tendon repair. ${ }^{2,5,6,9,37,42,44}$

Five clinical trial studies, over the past eight years, have been conducted using the ASTM or GISTM technique. Sevier and colleagues ${ }^{40}$ conducted a trial of ASTM on a patient suffering for two years from chronic lateral epicondylitis. ${ }^{40}$ The patient's pain and disability significantly decreased with an increase in strength, and discontinued use of the tennis elbow brace. Wilson and colleagues ${ }^{49}$ performed ASTM on twenty patients with patellar tendinitis. Sixty percent of the traditional rehabilitation group symptoms and pain resolved with 100 percent of the ASTM group experiencing complete resolution of symptoms and pain. The remaining 40 percent of the patients from the traditional rehabilitation group, crossed-over to the ASTM group with 50 percent undergoing resolution of symptoms and pain. Hammer and $\mathrm{Pfefer}^{50}$ treated a patient diagnosed with subacute lumbar compartment syndrome using GISTM. After several treatments the patient was asymptomatic, had nonrestrictive active and passive range of motions in all directions, normal functional tests and a significant improvement in hamstring and right external rotator flexibility. Aspegren and colleagues ${ }^{51}$ used GISTM on a patient suffering from costochondritis. From the initial visit, there was a decrease in pain and a significant 
improvement in the Dallas Pain Questionnaire. ${ }^{51}$ Lastly, Burke and colleagues ${ }^{52}$

conducted a pilot study on twenty-two patients with carpal tunnel syndrome using both the GISTM and ASTM techniques. There was an increase in nerve conduction latencies, increased wrist strength, increased range of motion and had greater patient satisfactory ratings.

Despite case reports and clinical studies, there have been no experimental studies conducted on GISTM technique and the effects on chronic ankle instability. Most of the clinical studies and case reports that have been conducted have used animals and human subjects for lateral epicondylitis, ${ }^{40}$ patellar tendinopathy, ${ }^{49}$ rotator cuff tendinopathy ${ }^{36}$ and achilles tendinopathy. ${ }^{36,37,38,39,40,41}$ Only two studies involved ligaments. ${ }^{41,45}$ Melham completed a case report on chronic ankle pain and fibrosis using the ASTM technique and found pain ceased with activity, the subjects range of motion improved, surgical scar matured, and the excessive fibrotic connective tissue around the ankle softened and diminished. ${ }^{41}$ Loghmani and colleagues ${ }^{45}$ conducted a study on rats using the GISTM technique in an attempt to examine the biomechanical and histological effects of instrumented-assisted cross fiber massage (IACFM) on early and long-term ligament healing. Twenty-one rats received nine treatments over a four-week period and thirty received thirty treatments over twelve weeks. At four and twelve weeks the ligaments were pulled to tensile failure. At four weeks a 31 percent increase in strength was found with 34 percent stiffer ligament structure compared to the untreated ligament. At twelve weeks a 15.4 percent stiffer ligament was found. Histological exams of the ligaments treated with IACFM were found to have greater fibroblast proliferation and more regular collagen alignment at either four or twelve weeks. ${ }^{45}$ In spite of this, no study has 
furthered evaluated the effects of chronic ankle instability and the condition of the ligaments after such an injury.

Beneficial effects have been noted with dynamic postural balance over a fourweek period ${ }^{34,35}$ using chronic ankle instability subjects. Since only a case report exists in the literature for chronic ankle instability using Graston Instrument-Assisted Soft Tissue Mobilization, the intent of this study is to further evaluate the effects of Graston Instrumented-Assisted Soft Tissue Mobilization and dynamic postural balance on improvements in healing and function. Therefore, the present study aims to investigate the affects of Graston Instrumented-Assisted Soft Tissue Mobilization (GISTM) technique and its influence on improving range of motion, neovascularization, collagen alignment, pain and disability in individuals experiencing chronic ankle instability.

\section{METHODS}

This study was a $2 \times 3$ factorial design for three of the dependent variables and a 2 $\times 3 \times 3$ and $2 \times 3 \times 4$ for two dependent variables. The independent variables of this study were time and group. The testing existed on two levels (pre and post). There were three levels that existed for group (GISTM/dynamic balancing-training program (GISTM/DBT), GISTM-Sham/dynamic balancing-training program (GISTM-S/DBT) and control group (CG)). The dependent variables consisted of six different measures that were performed by the subjects. Quantitative dependent variables included: FAAM scores, FAAM Sport scores, VAS measurement, ankle ROM degrees, and SEBT measurements. Qualitative dependent variable included US codes. The $2 \times 3$ factorial design was used for the FAAM, FAAM Sport, and VAS. Ankle ROM was a 2 × 3 x 4 factorial design. A $2 \times 3 \times 3$ factorial design was used for the SEBT to measure time, 
group, and direction.

Subjects

Thirty-six subjects $(\mathrm{n}=36 ; 5$ female, 31 male; age $=17.771 \pm 1.972$ years; ht $=69.403 \pm 5.775$ inches; 30 right, 6 left foot; 28 six sessions, 8 seven sessions of completed rehab) from the starting forty-five subjects completed the study. Eleven subjects were randomly assigned to the control group/DBT, 12 to the GISTM-S/DBT group and 13 to the GISTM/DBT group. Nine subjects dropped out of the study (3 suspensions, 1 sick, 2 suffered ankle injuries during competition, and 3 did not complete $75 \%$ of the rehabilitation sessions).

The subjects were obtained from North Central High Schools in West Virginia, DI Mid-Atlantic University, and an area recruitment ad. Subjects completed an ankle instability questionnaire (demographic questionnaire) that contained criteria for chronic ankle instability classification. Inclusion criteria for this study included a history of more than one ankle sprain and residual symptoms, frequent giving way of the ankle (at least one month) as quantified by four or more "yes" responses on the subject demographic questionnaire (which included questions from the Ankle Instability Instrument ${ }^{54}$ ), pain, feeling of instability, decreased function, no history of lower-extremity surgery, and no balance disorders, neuropathies, diabetes, or other conditions known to affect balance. If a subject reported bilateral ankle instability, the self-reported worse limb was used for analysis and training. Subjects were excluded if they had experienced an acute ankle sprain within six weeks of participation, if they had a history of surgery or fracture to either lower extremity, or if the stress x-ray revealed a grade III lateral ankle sprain. The University Office of Research Compliance approved this study. 
Instrumentation

Demographic questionnaire: The demographic questionnaire is a combination of questions that Hubbard et al. ${ }^{10,53}$ used as a criteria for functional ankle instability and Docherty et al. ${ }^{54}$ used in the Ankle Instability Instrument. The questions quantified the participants' functional ankle instability for inclusion or exclusion into the study.

FAAM ADL and FAAM Sport: The FAAM ADL and FAAM Sport are designed to meet the need for a self-reported evaluative instrument that comprehensively assesses physical function of individuals with musculoskeletal disorders of the leg, foot, and ankle. ${ }^{56}$ The FAAM is a region-specific self-report of function with two components just like the Functional Ankle Disability Index (FADI). ${ }^{55,56}$ The FAAM ADL is 21 -item questionnaire related to activities of daily living and the FAAM Sport incorporates eight more items that evaluate perceived disability due to foot or ankle injury in activities associated with physical activity and sport participation. ${ }^{55,56}$

Martin et al. ${ }^{56}$ reported that the FAAM ADL and FAAM Sport are reliable, valid, and responsive measure of self-reported physical function. Validity evidence was provided for test content, internal structure, score stability, and responsiveness. Test retest reliability was 0.89 and 0.87 for the ADL and Sports subscales, respectively. ${ }^{56}$ The minimal detectable change based on a 95 percent confidence interval was \pm 5.7 and \pm12.3 points for the ADL and Sports subscales, respectively. ${ }^{56}$ Two-way repeated measures ANOVA and ROC analysis found both the ADL and Sport subscales were responsive to changes in status $(\mathrm{p}<0.05) .{ }^{56}$ The minimal clinically important differences were 8 and 9 points for the ADL and Sport subscales. ${ }^{56}$ Guyatt responsive index and ROC analysis found the ADL subscale was more responsive than general measures of 
physical function while the Sports subscale was not. ${ }^{56}$ Then Carcia et al ${ }^{57}$ reported that for both the ADL and Sports subscales, FAAM scores were greater in healthy participants $(100 \pm 0.0$ and $99 \pm 3.5)$ than in subjects with $\mathrm{CAI}(88 \pm 7.7$ and $76 \pm 12.7 ; \mathrm{P}<.001) .{ }^{57}$

Similarly, for both ADL and Sports subscales, FAAM scores were greater in athletes who indicated that their ankles were normal $(98 \pm 6.3$ and $96 \pm 6.9)$ than in those who classified their ankles as either nearly normal, or abnormal $(87 \pm 6.6$ and $71 \pm 11.1 ; \mathrm{P}<.001){ }^{57}$ Carcia et $\mathrm{al}^{57}$ found relationships between FAAM scores and self-reported global ratings of function for both ADL and Sports subscales. Relationships were stronger when all athletes, rather than just those with CAI, were included in the analyses. ${ }^{57}$ Compared with other instruments, the FAAM ADL and FAAM Sport are measures specific to the leg, foot and ankle that show promise in quantifying function among a young, active population with chronic ankle instability. ${ }^{56,57}$

The directions consisted of instructions to answer every question with one response that most closely described their condition within the past week. ${ }^{55,56}$ If the activity in question is limited by something other than the foot or ankle, N/A is marked. Activity is rated as no difficulty at all (4 points), slight difficulty (3 points), moderate difficulty (2 points), extreme difficulty (1 point), unable to do (0 points), or N/A (not applicable). For pain related to the foot and ankle, patients select no pain (4 points), mild (3 points), moderate ( 2 points), severe ( 1 point), or unbearable ( 0 points) ${ }^{55,56}$ The FAAM scores are recorded as a percentage of 84 points. ${ }^{556}$ The FAAM Sport scores are recorded as a percentage of 32 points. ${ }^{556}$ Additionally, the patient is asked to rate their current level of function during usual ADLs and sport activities from 0-100 percent. ${ }^{56}$ 
VAS pain scale: Over the decades, the visual analogue scale (VAS) have come to be one of the most popular methods for measurement of pain intensity. ${ }^{58,59,60}$ The VAS consisted of a ten cm line anchored by two extremes of pain, usually "no pain" and "unbearable pain." $" 58,59,60,61$ The patient was asked to make a vertical mark through the line corresponding to the intensity of pain. ${ }^{58,59,60,61}$ The scale was scored by measuring the distance in mm from "no pain" to the patient's mark. ${ }^{58,59,60,61}$ Simple and reproducible, the VAS has been shown to yield reliable and valid pain estimates. Reliability coefficients exceeded 0.95 and validity correlations of 0.62 , respectively. ${ }^{62}$ Furthermore, several studies have demonstrated that VAS is usually more sensitive than traditional descriptive pain scales for detecting fine changes in pain levels. ${ }^{58,59,60}$

Of great significance, at least for research purposes, is the fact that the VAS provides ratio data and permits the use of parametric statistics. ${ }^{58,59,60,61}$ Test-retest reliability has been reported to be high $(\mathrm{ICC}=0.71-0.99)$ and concurrent validity $(0.71$ 0.78 ) has been found to be moderate for the VAS. ${ }^{58,59,60,61}$ The VAS is considered a strong, clinically useful, reliable and valid measure of pain intensity $58,59,60,61$

Goniometry: Visual estimation is commonly used in clinical practice and is very insufficient and unreliable. ${ }^{63,64,65,66}$ Menadue et al. ${ }^{63}$ stated that inter-observer reliability for visual estimation of active plantarflexion and dorsiflexion is very low. Using the goniometric measurement protocol for inversion and eversion, within session intraobserver reliability was high to very high ranging from $\mathrm{ICC}=0.82$ to 0.96 . No significant difference was found in ICC scores between position or directions of movement. ${ }^{63,64,65,66}$ Between-session intra-observer reliability ranged from low to high ( $\mathrm{ICC}=0.42$ to 0.80 ) but highest for inversion. ${ }^{1,64,65,66}$ Goniometric measurements of inversion were 
moderately well correlated between the prone and sitting positions. ${ }^{63,64,65,66}$ Overall, reliability of standardized measurement of ankle inversion and eversion range of motion was variable within observers, between observers and between-sessions.

Rome and Coweison ${ }^{64}$ examined intratester and intertester reliability for goniometric measurements of ankle dorsiflexion (ADF) and ankle plantar flexion (APF) active range of motion (AROM). For intratester reliability of measurements obtained with the universal goniometer (UG), intraclass correlation coefficients (ICC) for all physical therapists were $\mathrm{ICC}_{\mathrm{ADF}}=0.64-0.92$ (median, 0.825) and $\mathrm{ICC}_{\mathrm{APF}}=0.47-0.96$ (median, 0.865). Intertester reliability measurements obtained by universal goniometer were $\mathrm{ICC}_{\mathrm{ADF}}=0.28$ and $\mathrm{ICC}_{\mathrm{APF}}=0.25$. Visual estimation $(\mathrm{VE})$ was $\mathrm{ICC}_{\mathrm{ADF}}=0.34$ and $\mathrm{ICC}_{\mathrm{APF}}=0.48$. The parallel-forms intratester reliability obtained with UG and VE ranged from $\mathrm{ICC}_{\mathrm{ADF}}=0-0.94$ (median, 0.58) and $\mathrm{ICC}_{\mathrm{APF}}=0-0.86$ (median, 0.625). ${ }^{64}$ Thus, a goniometer can be used when making repeated measurements of ankle joint AROM. Considerable inconsistency exists when two or more examiners make repeated goniometric and visual measurements of ankle motion on the same subject.

Ultrasonography: A General Electric LOGIQ e portable ultrasound unit (GE Medical Systems, Milwaukee, WI) was used to evaluate collagen alignment and neovascularization for the ankle ligaments. The LOGIQ e is a 2.49-inch $\mathrm{x} 3.12$-inch $\mathrm{x}$ 11.7-inch laptop style console weighing approximately $10.1 \mathrm{lbs}$ with a fifteen-inch display screen with resolution color LCD. A one-probe port with micro-connector accompanies the LOGIQ e. The LOGICQ e has an intergrated HDD (80GB) and USB drive. 
Ligaments consist of dense connective tissue, somewhat similar to tendons. Many superficial ligaments are amenable to ultrasonography evaluation. The sonographic appearance differs slightly from tendons, with ligaments demonstrating as more compact, thickened and hypoechogenicity compared with the contralateral asymptomatic side. ${ }^{67}$ Hyperechoic appearance on longitudinal imaging is typically only a few millimeters in width ${ }^{67}$ However, a complete tear can be diagnosed when a hypoechoic gap or cleft is demonstrated through the substance of a ligament. ${ }^{67}$

Studies have found that ultrasonography is effective in diagnosing ankle ligament injuries, most notably those affecting the anterior talofibular ligament (ATFL). Van Dijk et al ${ }^{68}$ reported that ultrasonography was 92 percent sensitive and 64 percent specific for diagnosing injuries to the lateral ankle ligaments. Unfamiliarity of the technique, anatomy and pathology of assessing the lateral ankle complex can lead to a major limitation and inconvenience. Therefore, knowledge of the correct positioning of the ultrasonography probe is imperative.

Star Excursion Balance Test (SEBT): The star excursion and functional reach tests for dynamic postural control tests are superior to basic single leg stance (SLS) tests. ${ }^{69}$ The Star Excursion Balance Test simply tests an individual's dynamic postural control. The SEBT, first introduced by Gary Gray in 1995, is a functional balance test that uses a unilateral stance on the center of an asterisk (star) and a maximal reach down each of the asterisk's eight lines. ${ }^{69,70,71,72}$ The SEBT offers a simple, reliable, low-cost alternative to more expensive, refined instruments available today. ${ }^{73}$ An asterisk is made with lines extended out from the center at 45 degree increments on a grid. Each line has a specific name in relation to which leg is being tested (the leg the subject is standing on is 
the leg being tested). The eight directions are named anterolateral (AL), anterior (A), anteromedial (AM), medial (M), posteromedial (PM), posterior $(\mathrm{P})$, posterolateral $(\mathrm{PL})$, and lateral $(\mathrm{L})$ respective to the foot that is weight-bearing. ${ }^{69,70,71,72,73,74,75}$ For research and testing procedures in the clinic only the anterior (A), posteromedial (PM), and posterolateral (PL) directions can de used. These three directions have been found to assess unique aspects of dynamic postural control. ${ }^{35,76}$

The SEBT is placed directly on a non-slick floor using tape, three tape measures, and a protractor. The subject unilaterally stands in the center of the asterisk and maximally reaches with the contralateral leg down each line. ${ }^{73}$ The test requires three ${ }^{76}$ practice reaches in each of the three directions and three recorded test reaches. Each reach, or trial, should be held for one second for recording of measurements.

Each trial is measured from the center of the asterisk and the three trials are averaged and normalized to the subject's height or true leg length. A more general approach to compare SEBT excursions to is add the averages from each of the three directions and then multiply by the subject's height; this approach however, lacks the specificity of direction in which the subject is lacking dynamic postural control. Nevertheless, it provides an overall dynamic postural control evaluation to compare that of other subjects'.77

The further the reach, the greater the demand is on the dynamic postural control of the weight-bearing leg. ${ }^{18,69,70,71,72}$ The subject returns to the static unilateral stance position following each reach and remains there for 10-15 seconds before the next trial. During the SEBT, the individual's dynamic postural control constantly corrects as the center of mass migrates from over the base of support in all directions. ${ }^{78}$ Some factors to 
be considered when testing an individual using the SEBT are balance disorders, foot type, past injuries/surgeries, flexibility, and shoe condition. ${ }^{75,78}$

The star excursion balance test (SEBT) has been reported to have high reliability for testing dynamic postural control of those with and without functional ankle instability. ${ }^{18,70,71,72,79,80}$ Hertel et al. ${ }^{18}$ found intratester reliability of the SEBT between 0.78 and 0.96 . Olmsted et al. ${ }^{73}$ and McKeon et al. ${ }^{35}$ have both found the SEBT to be sensitive in detecting reach deficits between athletes with chronic ankle instability and healthy athletes. Validity for SEBT was also determined from Olmstead et al. ${ }^{73}$ and McKeon $^{35}$. Kinzey ${ }^{72}$ determined that the SEBT has moderate reliability for assessing dynamic balance and has an Intraclass Correlation Coefficient (ICC) of 0.86 to 0.98 for assessing dynamic balance.

Procedures

Subjects were contacted and asked to attend a meeting where they were provided with a Parental Consent Form and Assent Form if under age 18, a Consent Form, an Authorization to Use or Disclose Protected Health Information Form, and a subject Demographic Questionnaire. The study was described to the potential subjects to make an informed decision about whether to participate or not. Any questions from the potential subject pool were answered and explained. The potential subjects were then asked to fill out the Parental Consent Form (Table C1), Assent Form (Table C2), or Consent Form (Table C3) the Authorization to Use or Disclose Protected Health Information Form (Table C4), and Subject Demographic Questionnaire (Table C5) truthfully and to the best of their ability. The demographic questionnaire demonstrated the subjects' eligibility by assessing chronic ankle instability. The subjects answered 
several questions concerning injury background past and present with the involved ankle. The researcher reviewed the forms for completeness to note if the subjects met the inclusion criteria, and not the exclusion criteria. Subjects were excluded if answering "yes" to the following questions: have you had any fractures (breaks) in either of your ankles, have you had any surgeries in either of your lower extremities, have you ever experienced a sensation of your ankle "giving way" $>$ than 3 months ago, and are you currently involved in a "formal" rehabilitation program for the affected ankle. Also if the subject answers "no" to the following question have you ever sprained an ankle, were excluded. If the subjects meet the inclusion criteria, they were contacted by the researcher to schedule a time to complete the pre-test FAAM ADL and FAAM Sport questionnaire, VAS, ultrasonography images, range of motion measurements, and to perform the Star Excursion Balance Test.

Times were established for subjects to meet with the researcher two times a week over four weeks for approximately 45 minutes per session for treatment, balance-training program, or a combination of the two. The injured ankle was used for the treatment/training sessions. The Demographic Questionnaire and special tests determined the injured ankle. All exercises were performed at a North Central High School in WV to serve as an environmental control. The primary researcher administered and supervised all treatment, testing and exercising sessions. At the conclusion of the last exercise session, post-test measurements for the FAAM ADL, FAAM Sport, VAS, goniometry, ultrasonography images, and SEBT tests were obtained. The post-test was performed to the exact specifications as the pre-test, and was completed within the week following the final treatment/training session of the fourth week. 
Pre- and Post-Testing

FAAM ADL and FAAM Sport: The subject completed the FAAM ADL and FAAM Sport (Table C6). The subjects were asked to answer the FAAM ADL 21-item questionnaire with one response that most clearly described their condition within the past week. If the activity in question was limited by something other than the foot/ankle, N/A is marked. And rated the current level of functional activity during ADLs from 0100 percent. Upon completion, the subjects answered the FAAM Sport 8-item questionnaire with one response that most clearly described the condition within the past week as well. If the activity in question was limited by something other than the foot/ankle, N/A is marked. And rated the current level of functional activity during sports from 0-100 percent. The primary researcher recorded the FAAM ADL scores as a percentage of 84 points and function percentage and the FAAM Sport scores as a percentage of 32 points and function percentage.

VAS pain scale: The subject made a mark on the $10 \mathrm{~cm}$ line that accurately described pain level at that instant on the $10 \mathrm{~cm}$ line. The primary researcher then measured the mark going from left to right in millimeters $(\mathrm{mm})$. The mean between the pre- and posttreatment measurements determined the subjects' resulting pain level. Refer to Table C7 for a more precise step-by-step protocol of the VAS pain scale.

Ultrasonography: The subjects' identification number was entered into the computer to create a folder of the subjects' images. The subjects were instructed to lie in the supine position with the subjects' knee in extension on the treatment table. The ankle was plantarflexed and inverted with the probe in a slightly oblique position from the malleolus toward the forefoot. ${ }^{68}$ The first image, the ATF ligament was stilled and saved 
to the subject folder. The color doppler/power doppler was turned on, with the same subject and probe placement. An image was stilled and saved in the subject folder of the area over the ATF ligament. The subjects were then instructed to remain in the supine position with the knee straight on the treatment table. Ankle placement for the $\mathrm{CF}$ ligament was dorsiflexed and inverted, the probe was placed slightly in a posterior oblique direction toward the calcaneus. ${ }^{68}$ The image of the CF ligament was stilled and saved in the subject folder. This was followed by color doppler/power doppler with the same subject and probe placement. Again the image was stilled and saved in the subject folder. (Table C9)

Ankle range of motion: Ankle range of motion was measured in degrees using a goniometer. The subjects were instructed to sit, with the knee flexed off the end of a treatment table for both plantarflexion and dorsiflexion. The goniometer was placed with the stationary arm along the midline of the fibula from the fibular head to lateral malleolus, and the movable arm was along the midline of the fifth metatarsal. The subject was asked to plantarflex the ankle as far as possible; after the recording the measurement, the subject dorsiflexed the ankle as far possible. For both inversion and eversion the subjects were instructed to lay prone. The tibia was stabilized in the sagittal plane. Subjects were instructed to invert and evert the calcaneus as far as possible. The goniometer for both inversion and eversion was placed with the stationary arm in alignment with the midline of the leg, and the movable arm aligned with the midline of the calcaneus. All data was recorded to the nearest degree.

Star Excursion Balance Test (SEBT): The subjects' true leg length was measured and recorded to normalize the data. The SEBT was performed with the subjects standing in 
the middle of a grid formed by eight lines extending out at $45^{\circ}$ from each other. The participant was asked to reach as far as possible along the anterior (A), posteromedial (PM), and posterolateral (PL) lines, make a light touch on the line, and return the reaching leg back to the center, while maintaining a single-leg stance with the other leg in the center of the grid.

Subjects were instructed to make a light touch on the ground with the most distal part of the reaching foot and return to a double-leg stance without allowing the contact to affect overall balance. The terminology of excursion directions is based on the direction of reach in relation to the stance leg (see Figure $\mathrm{C} 1$ ). When reaching in the lateral and posterolateral directions, subjects reached behind the stance leg to complete the task.

Subjects were allowed to practice reaching in each of the three directions three times to minimize the learning effect. ${ }^{76}$ Following a 5-min rest period, subjects performed three trials in each of the three directions. The subjects then randomly drew one of three index cards, which determined the starting direction. All subjects began with the involved stance leg in the center of the grid depending on the functionally unstable ankle. The primary researcher recorded each reach distance with a mark on the tape as the distance from the center of the grid to point of maximum excursion by the reach leg. The trial was discarded and repeated if the primary researcher felt the subject was using the reaching leg for a substantial amount of support at any time, has removed the foot from the center of the grid, or was unable to maintain balance on the support leg throughout the trial.

Interventions

Dynamic Flex Band flexibility warm-up routine: The dynamic Flex Band flexibility warm-up provided vital forces necessary to assist in remodeling collagen ${ }^{40}$ in individuals 
with chronic ankle instability. The dynamic warm-up routine incorporated the lower extremity as a whole, due to the movements of the dynamic balance-training program.

The dynamic warm-up was approximately ten minutes. The warm-up started with the ankles (inversion/eversion), gastrocnemius, achilles, hamstring, groin, I-T Band, and concluded with the quadriceps/hip flexor stretches. The routine was specific in that the ankles (inversion/eversion), gastrocnemius and achilles stretches were all done in sequence on one foot initially then completed on the opposite foot. After the Ankle Work $^{82}$ for both legs has been concluded, the subject subsequently moved onto the hamstring, groin, I-T Band, and quadriceps/hip flexor stretches. Once again one leg was completed before subsequently moving to the other leg. (Table C11 and Figures C2-8).

Rehabilitation Program: McKeon et al. ${ }^{35}$ developed a four-week progressive dynamic balance-training program for those with chronic ankle instability. He incorporated single-limb hops to stabilization, hop to stabilization and reach, unanticipated hop to stabilization, and single-limb stance activities into a rehabilitation program. All the exercises are designed to challenge the subjects' ability to maintain a single-limb stance while performing various balance activities. ${ }^{35}$ More specifically, the program challenges recovery of single-limb balance efficiently after a disturbance and effectively developed natural execution to accomplish movement goals. ${ }^{35}$ These innovative activities promotes the restoration of functional variability within the sensorimotor system with environmental constraints. ${ }^{35}$

The first of four exercises were single-limb hops to stabilization. Subjects performed ten repetitions per direction, anterior/posterior, medial/lateral, anterolateral/posteromedial, and anteromedial/posterolateral (see Figures C9-10). Each 
repetition consisted of a hop from the starting position to the target position at 18,27 , or 36 inches. After stabilizing balance in a single-limb stance, subject hopped in the exact opposite direction back to the starting position and stabilized in the single-limb stance. Subjects did not advance to the next level until the ten repetitions were error-free. Errors were determined on the basis of the following: touching down with the opposite limb, excessive trunk motion ( $>30^{\circ}$ lateral flexion), removal of hands from hips during hands on hips activities, bracing the nonstance limb against the stance limb, and missing the target.

The five-repetition hop to stabilization and reach exercise was a combination of the previously mentioned exercise. However, after stabilization in the single-limb stance, the subject reached back to the starting position. Subjects hopped, stabilized, and reached back to the starting position, then hopped back to the starting position and reached to the targeted position. A subjects' advancement to the next level were achieved when there was five error-free repetitions. Errors were determined by all errors associated with hop to stabilization and by using the reaching leg for substantial amount of support during the reaching component.

Both the hop to stabilization and hop to stabilization and reach have seven levels of difficulty. The progression began with an 18, 27 or 36-inch hop using the arms to aid in stabilizing balance after landing, to an 18, 27, or 36-inch hop with hands on hips while stabilizing balance after landing. The seventh and final level consisted of a 36-inch hop from a 6-inch platform.

The third exercise was an unanticipated hop to stabilization with the subjects standing in the middle of a nine-marker grid (see figure C11). A randomized, sequence of 
numbers was displayed on a computer screen in front of the subjects. Each number corresponded to a hop target position. As the progression of numbers changed, subjects hopped to the new target position. The hop to stabilization rules applied for this activity; however, in this case, subjects were allowed to use any combination of hops (AP, ML, $\mathrm{AM} / \mathrm{PL}$, or $\mathrm{AL} / \mathrm{PM}$ ) to accomplish the goal of moving through the sequence error-free. As a subject developed proficiency, the amount of time per move was reduced. In each session, subjects performed three sequences of numbers.

The unanticipated hop to stabilization also had seven levels of progression. As previously stated advancement was not made until error-free. The subject was given five, three, or one second(s) per move, with each level of progression. As the subject progressed to completion of all moves within one second without error, a foam pad was placed on one of the numbers during the sequence. The subject then continued the progression at the same level of intensity. If the course was not completed error-free, the error-free time constraint was increased to the level below. For the fifth level, a step was added to an additional number. At level six, an additional foam pad was added to one of the numbers, resulting in two foam pads and one step. An additional step was included in level seven, resulting in two foam pads and two steps. Errors were determined as touching down with the opposite limb, excessive trunk motion ( $>30^{\circ}$ lateral flexion), removal of hands from hips during hand on hips activities, bracing the nonstance limb against the stance limb, and missing the target.

The fourth and final exercise was the single-limb stance. Subjects performed three repetitions of a single-limb stance. Each activity (eyes open and eyes closed) consisted of seven levels of difficulty. In the single-limb eyes opened stance, arms were across the 
chest while standing on hard floor (60 seconds). The next progression was with the arms across the chest while standing on a foam pad (30,60, or 90 seconds). The subject then advanced to a ball toss on a foam pad with the arms across the chest. The progression continued with 20 throws with 6-lb medicine ball for 30 seconds. Time was increased to 60 seconds and finally 90 seconds. The final advancement was a single-limb stance with eyes closed and arms out while standing on a hard floor ( 30 seconds), to arms across the chest while standing on a hard floor ( 30 and 60 seconds). The exercise progressed to the arms out on a foam pad ( 30 seconds), and finally the arms across the chest on a foam pad for 30, 60, and 90 seconds. As previously stated advancement was not made until errorfree. Errors were determined by subjects touching down with opposite limb, excessive trunk motion ( $>30^{\circ}$ lateral flexion), removal of arms from across the chest during specified activities, and bracing the nonstance limb against the stance limb.

Graston Instrument-Assisted Soft Tissue Mobilization (GISTM): Table C14 gives specific instructions on subject and primary researcher positioning. The subjects were instructed to lie prone with the foot over the end of the treatment table. Emollient was rubbed onto the posterior lower leg. The hands were then washed. The primary researcher stood next to the subject. The primary researcher started with the GT-4 and GT-5 tool. Using a two-hand hold with GT-4, a sweeping motion over the plantar fascia and heel pad was used. A sweep and fan technique using GT-5 was used for the gastrocnemiussoleus complex starting proximal to the achilles tendon insertion up to the popliteal fossa. The ankle was assessed in a neutral position and in dorsiflexion to vary the tension of the tissue, which affected the instrument's depth of penetration. The GT-3 tool was used next with a pencil grip, to localize any restrictions within the gastrocnemius-soleus complex, 
achilles tendon, and plantar fascia. The GT-2 or GT-3 tool was used with a two-hand hold, placing additional pressure over the knob of GT-2. The soft tissue lying in the groove between the achilles tendon and the fibula were mobilized laterally and medially, between the tendon and the tibia. Moving to a one-hand hold, the GT-2 or GT-3 tool was used. Using the GT-2 knob to mobilize the plantar fascial strips were mobilized from the calcaneus to the metatarsal head and back. To vary the fascial tension, the toes were flexed and extended. Lastly, the GT-2 tool were used with a one-hand hold using the curve to work between the toes.

The subjects were instructed to lie supine with the foot over the end of the treatment table. With the primary researcher standing next to the subject, emollient was rubbed on the anterior lower leg. Again the hands were washed. Using the GT-5 tool with a two-hand hold, the dorsum of the foot was scanned moving proximally to the anterior tibialis. The GT-4 tool with a two-hand hold was used to sweep the dorsum of the foot and anterior tibialis to isolate restrictions. Better isolation of the adhesion can be achieved by reversing the stroke pattern. Once the area and restrictions were identified the GT-2 or GT-3 tool with a two-hand hold was used to work around the medial and lateral malleolus. After mobilization of the first metatarsal head, the GT-2 or GT-3 tool was used with a two-hand hold. The GT-2 or GT-3 tool was used with a one-hand hold. The knob of GT 2 or GT-3 was used to mobilize the soft tissue around the talocrural joint and distal tibiofibular joint. Using the other hand, dorsiflexion/plantarflexion, inversion/eversion and 1st toe flexion/extension was performed. This allowed a different depth of instrument penetration. The GT-2 or GT-3 tool was used with a two-hand hold, sweeping up and down the medial and lateral aspect of the tibia using the knob of GT-2 
so as to release fascial compartments attached to this bone. The focus was to isolate restrictions.

The subject was finally instructed to move to a side lying position with a pillow to support the limb. The primary researcher was sitting on a stool at the side of the subject. Depending on the size of the leg, GT-3, GT-4 or GT-2 tool with a pencil grip was used to strum, fan, sweep, and J-stroke on the peroneals as needed. After the GISTM treatment, the tools were cleaned with SaniCide, a hard surface disinfectant.

Statistical Analysis

Descriptive statistics included means and standard deviations. Four separate 2 × 3 repeated measures ANOVA (time x group) were analyzed for the FAAM ADL scores, FAAM Sport scores, and VAS measurements. A $2 \times 3 \times 3$ quantitative repeated measures ANOVA (time x group $\mathrm{x}$ direction) was used for the SEBT measurements, while a $2 \times 3 \times 4$ repeated measures ABOVA (time x group x movement) was used for ROM measurements. Ultrasonography qualitative results for neovascularization and collagen alignment was compared to the baseline measurements. Chi-Square analysis was originally to be used for comparisons of pre-post tests, but due to the variability of images was not used. Alpha level used a priori of $\mathrm{p}<0.05$ with a bonferroni correction of $\mathrm{p}=0.01$ for all analysis. Cohen's D measures of effect size were determined by calculating the mean difference between groups (DBT-GISTM, DBT-Sham GISTM, control) and time (pre- and post-test) and dividing it by the reference SD (pretest or control). The strength of effect sizes were determined as small $(\leq 0.04)$, moderate $(0.41-0.7)$, and large effects $(\geq 0.71)$. Percent change scores were also evaluated to examine the amount of change that occurred within group. 


\section{RESULTS}

Disability Questionnaires and Pain Scale

Descriptive statistics for the pre-test and post-test data by group including the

Foot and Ankle Ability Measure (FAAM), FAAM Sport, and Visual Analog Scale (VAS) can be found in Table D1. For the FAAM there was a significant difference for the main effect of time $\left(\mathrm{F}_{1,33}=47.963, \mathrm{P}=0.01, \mathrm{ES}=0.592,1-\beta=1.00\right)$ with post-test scores higher than pre-test (Tables D1-2). There was no significant interaction for time by group $\left(F_{2,33}=0.051, P=0.950, E S=0.003,1-\beta=0.012\right)$, nor a significant main effect for group $\left(\mathrm{F}_{2,33}=0.051, \mathrm{P}=0.950, \mathrm{ES}=0.003,1-\beta=0.012\right)$. However, the Control/Dynamic BalanceTraining (C/DBT), Graston Instrumented-Assisted Soft Tissue MobilizationSham/Dynamic Balance-Training (GISTM-S/DBT), and Graston Instrumented-Assisted Soft Tissue Mobilization/Dynamic Balance-Training (GISTM/DBT) groups, percent change scores increased 10.7, 11.5, and 10.2 percent, respectively. With the GISTMS/DBT group improving more than the C/DBT and GISTM/DBT groups.

For the FAAM Sport there was a significant difference for the main effect of time $\left(\mathrm{F}_{1,33}=18.377, \mathrm{P}<0.001, \mathrm{ES}=0.358,1-\beta=0.932\right)$ with post-test scores higher than pre-test (Tables D1-2). There was no significant interaction for time by group $\left(\mathrm{F}_{2,33}=2.153\right.$, $\mathrm{P}=0.132, \mathrm{ES}=0.115,1-\beta=0.187)$, nor a significant main effect for group $\left(\mathrm{F}_{2,33}=2.153\right.$, $\mathrm{P}=0.132, \mathrm{ES}=0.115,1-\beta=0.187)$. Percent change scores increased for the $\mathrm{C} / \mathrm{DBT}(5.3$ percent), GISTM-S/DBT (30.6 percent), and GISTM/DBT (31.1 percent) groups. With the GISTM/DBT group improving more than the C/DBT and GISTM-S/DBT groups.

For the VAS there was a significant difference for the main effect of time $\left(\mathrm{F}_{1,33}=\right.$ 55.564, $\mathrm{P}<0.001, \mathrm{ES}=0.590,1-\beta=1.000)$ with post-test scores higher than pre-test (Tables $\mathrm{D} 1-2)$. There was no significant interaction for time by group $\left(\mathrm{F}_{2,33}=0.215, \mathrm{P}=0.808\right.$, 
$E S=0.013,1-\beta=0.020)$, nor a significant main effect for group $\left(F_{2,33}=0.160, P=0.853\right.$, $E S=0.010,1-\beta=0.017)$. Percent change scores decreased for pre- to post-test for $C / D B T$ (4.2 percent), GISTM-S/DBT (20.8 percent), and GISTM/DBT (20.9 percent) groups. The GISTM/DBT and GISTM-S/DBT groups decreased the most with the C/DBT group decreasing the least.

Range of Motion (ROM)

Descriptive statistics for the pre-test and post-test data by group for ROM can be found in Table D1. There was a significant difference for the main effect of time $\left(\mathrm{F}_{1,33}=40.087, \mathrm{P}<0.001, \mathrm{ES}=0.548,1-\beta=1.000\right)$ as seen in Table D5. There was a significant difference between pre- and post-test for Dorsiflexion ( $\mathrm{t}=-6.895,-3.028,98 \%$ CI -4.098- -1.957, $\mathrm{P}=<0.001)$, Plantarflexion $(\mathrm{t}=-3.639,-4.382,98 \%$ CI -7.317- -1.447, $\mathrm{P}=0.001)$, Inversion $(\mathrm{t}=-2.958,-1.729,98 \% \mathrm{CI}-3.154--0.304, \mathrm{P}=0.006)$, and Eversion $(\mathrm{t}=-6.537,-1.292,98 \% \mathrm{CI}-1.773--0.810, \mathrm{P}=<0.001)$ movements. There was a significant difference for the main effect of movement $\left(\mathrm{F}_{3,33}=196.721, \mathrm{P}<0.001, \mathrm{ES}=0.856,1-\right.$ $\beta=1.000$ ). With significance for mean differences between Dorsiflexion and Plantarflexion (-13.271 $\left.{ }^{\circ}, 99 \% \mathrm{CI},-18.027--8.516, \mathrm{P}<0.001\right)$, Dorsiflexion and Eversion (11.634 ${ }^{\circ}, 99 \%$ CI, 9.853-13.414, $\left.\mathrm{P}<0.001\right)$, Plantarflexion and Inversion $\left(15.092^{\circ}, 99 \%\right.$ CI, 10.404-19.781, P<0.001), Plantarflexion and Eversion (24.905 , 99\% CI, 20.19329.618, $\mathrm{P}<0.001$ ), and Inversion and Eversion (9.813 $, 99 \% \mathrm{CI}, 8.845-10.782, \mathrm{P}<0.001)$. There was no significant interaction for time by group $\left(\mathrm{F}_{2,33}=3.720, \mathrm{P}=0.035\right.$, ES $=0.184$, $1-\beta=0.379)$, movement by time $\left(F_{3,33}=4.331, P=0.045, E S=0.116,1-\beta=0.275\right)$, movement by group $\left(\mathrm{F}_{6,33}=0.592, \mathrm{P}=0.736, \mathrm{ES}=0.035,1-\beta=0.082\right)$, and time by movement by group $\left(\mathrm{F}_{6,33}=1.564, \mathrm{P}=0.225, \mathrm{ES}=0.087,1-\beta=0.124\right.$. There was no significant main effect of 
group $\left(\mathrm{F}_{2,33}=0.498, \mathrm{P}=0.612, \mathrm{ES}=0.029,1-\beta=0.036\right)$ even though the percent change scores were evident from pre- to post-test for all movements which can be further evaluated in Table D8. The GISTM/DBT group demonstrating the most improvement in Dorsiflexion, Plantarflexion and Inversion followed by the C/DBT and GISTM-S/DBT groups; and with the GISTM-S/DBT group demonstrated the most improvement in Eversion followed by the C/DBT and GISTM/DBT groups. (Refer to Table D8). Star Excursion Balance Test (SEBT)

Descriptive statistics for the pre-test and post-test data by group for (SEBT) can be found in Table D1. There was a significant difference for the main effect of time $\left(\mathrm{F}_{1,33}=58.493, \mathrm{P}=0.000, \mathrm{ES}=0.639,1-\beta=1.000\right)$ as seen in Table D9. There was a significant difference between pre- and post-test for Anterior $(\mathrm{t}=-5.490,-8.011,98 \% \mathrm{CI}$ 11.568- -4.454, $\mathrm{P}<0.001)$, Posteromedial $(\mathrm{t}=-5.769,-6.625,98 \%$ CI $-9.425--3.825$, $\mathrm{P}<0.001)$, and Posterolateral $(\mathrm{t}=-6.237,-7.553,98 \% \mathrm{CI}-10.505--4.601, \mathrm{P}<0.001)$. There was a significant difference for direction $\left(\mathrm{F}_{1,33}=71.355, \mathrm{P}<0.001, \mathrm{ES}=0.684,1-\beta=1.000\right)$ with significance for mean differences between Anterior and Posteromedial (4.562in, 99\% CI, 1.146-7.978, $\mathrm{P}=0.001$ ), Anterior and Posterolateral (17.339in, 99\% CI, 12.48122.196, $\mathrm{P}=0.001$ ), and Posteromedial and Posterolateral (12.777in, 7.055-18.499, $\mathrm{P}<0.001)$. There was no significant interaction for time by group $\left(\mathrm{F}_{2,33}=1.992, \mathrm{P}=0.152\right.$, $\mathrm{ES}=0.108,1-\beta=0.169)$, direction by group $\left(\mathrm{F}_{4,33}=0.412, \mathrm{P}=0.800, \mathrm{ES}=0.024,1-\beta=0.042\right)$, time by direction $\left(\mathrm{F}_{2,33}=0.504, \mathrm{P}=0.606, \mathrm{ES}=0.015,1-\beta=0.038\right)$, and time by direction by group $\left(\mathrm{F}_{4,33}=1.942, \mathrm{P}=0.114, \mathrm{ES}=0.105,1-\beta=0.307\right)$. There was no significant main effect of group $\left(\mathrm{F}_{2,33}=0.291, \mathrm{P}=0.749\right.$, $\left.\mathrm{ES}=0.017,1-\beta=0.024\right)$ even though percent change scores from pre- to post-test were evident for the three directions. The GISTM/DBT 
group demonstrating the most improvement in Anterior and Posteromedial followed by C/DBT and GISTM-S/DBT groups; and with the GISTM-S group demonstrating the most improvement in Posterolateral followed by C/DBT and GISTM/DBT groups. (Refer to Table D12).

Ultrasonography

Descriptive statistics for the pre-test and post-test data for ultrasonography of the anterior talofibular ligament (ATF) and calcaneofibular ligament (CF) were unavailable due to variability of images for the two ligaments. Only three pre and post-test images were available for visual comparison, two for the GISTM group and one from the GISTM-Sham group. However, based on visual analysis no apparent differences in collagen alignment and neovascularization were evident.

\section{DISCUSSION}

The present study found that a 4-wk Graston Instrumented-Assisted Soft Tissue Mobilization (GISTM) treatment and a dynamic balancing-training (DBT) program improved self-reported function, range of motion (ROM), and dynamic postural control (Star Excursion Balance Test (SEBT)) in individuals experiencing chronic ankle instability. There was a significant difference for test with FAAM, FAAM Sport, VAS, ROM, and SEBT with post-test results greater except for VAS, which decreased. There were also significant differences for movement (ROM) and direction (SEBT). While there were no significant differences between experimental groups, an increase was evident in all pre-test to post-test results for all groups with the GISTM/DBT group showing the best percent change improvements followed by the C/DBT and GISTMS/DBT groups. 
It was hypothesized that the GISTM/DBT group would be affected differently compared to the other two groups, C/DBT and GISTM-S/DBT groups for the Foot and Ankle Ability Measures (FAAM), FAAM Sport, Visual Analog Scale (VAS), ROM, and Star Excursion Balance Test (SEBT). This was not supported by the results, as there was not a significant difference between groups for the FAAM, FAAM Sport, VAS, ROM, or SEBT. However, the present data does suggest that all three groups resulted in significant improvements during time, movements, and direction. There were differences evident for pre- and post-test results for all tests. The GSITM-S/DBT group demonstrated the most improvement followed by the $\mathrm{C} / \mathrm{DBT}$ and GISTM/DBT groups in percent changes scores for the FAAM. The GISTM/DBT group demonstrated the most improvement followed by the GISTM-S/DBT and C/DBT groups in percent change scores for the FAAM Sport and the VAS test. Furthermore, for ROM and SEBT, the GISTM/DBT group demonstrated the most improvement followed by the C/DBT and GISTM-S/DBT groups in percent change scores for the movement of dorsiflexion (DF), inversion (INV), and the anterior (A) direction. The GISTM/DBT group demonstrated the most improvement followed by the C/DBT and GISMT-S/DBT groups in percent change scores for the movement of plantarflexion (PF) and the posteromedial (PM) direction. And finally, the GISTMS/DBT group demonstrated the most improvement followed by the C/DBT and GISTM/DBT groups in percent change scores for the movement of eversion (EV) and the posterolateral (PL) direction. There were no significant interactions for any of the five tests. However, there was a significant main effect with movement for ROM, and direction for the SEBT. Overall, the GISTM/DBT group demonstrated the most improvement in percent change scores than the C/DBT and GISMT-S/DBT groups. 
Despite the lack of significant differences between the experimental groups, the results of this study are somewhat encouraging. Although the GISTM treatment and the DBT program prescribed in this study have not been most widely used in rehabilitation settings, there is a sound rationale for incorporating the two in rehabilitation programs for athletes experiencing chronic ankle instability. Within a brief treatment period of four weeks, the GSITM/DBT group demonstrated improvement in sport disability, pain, ROM with dorsiflexion, plantarflexion, and inversion, along with the anterior and posterior medial directions for the SEBT when compared to the C/DBT and GISTM-S/DBT groups. While no optimal treatment program length has been recommended in the literature, it seems reasonable to conclude that a longer treatment period may have resulted in additional changes. It was felt that ankle flexibility and sensorimotor facilitation, along with the disability perceived by the subjects were enhanced with the treatment program including GISTM and dynamic balance-training exercises. Disability and Pain

While other studies using the Foot and Ankle Disability Index ${ }^{35}$ questionnaire, also demonstrated improvements from pre- to post-testing, this is one of the first to use the FAAM and FAAM Sport for quantitative disability data. Martin et al. ${ }^{56}$ reported that the FAAM activities of daily living (ADL) and FAAM Sport are reliable, valid, and responsive measure of self-reported physical function. Carcia et al. ${ }^{57}$ reported that for both the ADL and Sports subscales, FAAM scores were greater in healthy participants than in subjects with chronic ankle instability. The present study used only subjects who were experiencing chronic ankle instability (CAI), therefore, there was no available data on healthy subjects versus CAI subjects. 
For both ADL and Sports subscales, FAAM scores were greater in athletes who indicated that their ankles were normal than in those who classified their ankles as either nearly normal, or abnormal. ${ }^{57}$ However, the present study did not focus on how athletes classified their ankle function pre- and post-test.

A parallel disability questionnaire to the FAAM and FAAM Sport would be the Foot and Ankle Disability Index (FADI) and FADI Sport. McKeon et al. ${ }^{35}$ found a significant interaction with group by time for the FADI and FADI Sport scores. However, post hoc tests revealed no significance between pre-test measures for the FADI and the FADI between groups. ${ }^{35}$ This was also noted in the present study in that there were no differences between groups. Why this occurred is that traditionally, balance training involves single-limb stance activities on stable and unstable surfaces. Although selfreported improvements in functional status have been demonstrated in response to balance training, there is conflicting evidence that postural control improvements occur as a result of balance training in individuals with CAI. ${ }^{35}$ Rozzie et $\mathrm{al}^{83}$ reported similar results to McKeon et $\mathrm{al}^{35}$ in that improvements on Ankle Joint Functional Assessment Tool when comparing a group with CAI to a group of healthy controls who underwent the balance-training program. Rozzie et $\mathrm{al}^{83}$ found that individuals who underwent the 4wk of balance-training on the Biodex Stability System had improvements in self-reported function, regardless of group membership. This relates to the present study as the subjects in all three groups were experiencing CAI and improvement regardless of group membership as well.

Balance-training enhances the sensorimotor system but also the neuromuscular system. With CAI individuals there is decreased stability and function, balance-training 
will help reeducate the sensorimotor and neuromuscular systems to react to appropriately when protuberances occur. Therefore, the reeducation of both systems no matter CAI or healthy in disability and function will improve due to both systems fine tuning strength and endurance.

It is postulated that the repair process is similar in function with chronic injuries to that of acute injuries. When a ligamentous soft tissue injury occurs, one of the damaging mechanisms is scar tissue formation. To assist in removal of excessive scar tissue and the healing process, a type of soft tissue mobilization must be used. Augmented Soft Tissue Mobilization (ASTM) and GISTM decrease the number of scar tissue adhesions thus increasing function and decreasing pain which improves functional stability overall. ASTM introduces a more controlled amount of microtrauma into an area of excessive scar tissue, therefore, the response of the tendon to this microtrauma could involve augmentation of the healing process. ${ }^{36,37,38,39,40,41}$ GISTM, another variation of this technique, also was shown to increase the fibroblast response with the controlled movement of the instruments. ${ }^{2,5,6,9,37,42,44}$ Although most of the original research has been on tendons using animal and human subjects, there have been two studies reported using $\mathrm{ASTM}^{41}$ and $\mathrm{GISTM}^{45}$ on ligaments.

Five clinical trial studies, over the past eight years, have been conducted using the ASTM or GISTM technique. Sevier and colleagues ${ }^{40}$ conducted a trial of ASTM on a patient suffering for two years from chronic lateral epicondylitis. ${ }^{40}$ The patient's pain and disability significantly decreased with an increase in strength, and discontinued use of the tennis elbow brace. Wilson and colleagues ${ }^{49}$ performed ASTM on twenty patients with patellar tendinitis. Sixty percent of the traditional rehabilitation group symptoms and pain 
resolved with 100 percent of the ASTM group experiencing complete resolution of symptoms and pain. The remaining 40 percent of the patients from the traditional rehabilitation group, crossed-over to the ASTM group with 50 percent undergoing resolution of symptoms and pain. Hammer and $\mathrm{Pfefer}^{50}$ treated a patient diagnosed with subacute lumbar compartment syndrome using GISTM. After six treatments the patient was asymptomatic, had nonrestrictive active and passive range of motions in all directions, normal functional tests and a significant improvement in hamstring and right external rotator flexibility. Aspegren and colleagues ${ }^{51}$ used GISTM on a patient suffering from costochondritis. From the initial visit, there was a decrease in pain and a significant improvement in the Dallas Pain Questionnaire. ${ }^{51}$ Lastly, Burke and colleagues ${ }^{52}$ conducted a pilot study on twenty-two patients with carpal tunnel syndrome using both the GISTM and ASTM techniques. There was an increase in nerve conduction latencies, increased wrist strength, increased range of motion and had greater patient satisfactory ratings.

Like all five clincial trial studies, the present study also observed a decrease in disability and pain. Therefore, even though there was no objective data to confirm the benefits of the dynamic balance-training program with GISTM treatment despite the percent change scores, all the subjects felt as if they had improved. To some individuals this may be more important than an increase in range of motion. Concluding that the subject's perspective on their current state is more important than actual objective measure of improvement.

A very stringent $P$ value of 0.01 as well as the small sample size might have affected group differences. However, the similarity of the subjects at baseline despite 
group membership may be the main reason. The majority of the subjects, 72 percent, were 17 years old and in-season sport athletes. Therefore, they would be similar in growth, maturity, and fitness level. Also the majority of subjects with chronic ankle instability had a Grade 1 ankle sprain confirmed by special tests consisting of the anterior drawer, talar tilt inversion, and medial subtalar glide. Although not verified by ultrasonography, only a small to minimal amount of damage had been sustained by the ligament structures. Thus, all subjects experienced the changes evident following treatment.

Range of Motion

The present study focused on between group differences for pre- and post-test. All ROM measurements improved pre- and post-test despite the treatment group assignment, thus indicating why there was not a difference between groups. In addition, all four ROM movements including DF, PF, INV, and EV demonstrated improvement when comparing the difference between movements. From case reports and pilot studies, an increase in ROM has occurred following GISTM treatment. In the present study post-test measurements were higher than pre-test for ROM. Without ultrasonography it can not be concluded with certainty that the increase may have been due to the GISTM treatment removing restrictions or the DBT enhancing the sensorimotor and neuromuscular systems upon jumping and landing.

The GISTM treatment for both GISTM and GISTM-S groups may have stimulated the mechanoreceptors. It is well published in literature references that high frequency vibrations stimulate mechanoreceptors; therefore, the GISTM-S treatment even though the tools were barely gliding over the skin may have elicited the 
mechanoreceptors, which in turn decreased the response of the nocioreceptors and in turn resulted in a decrease in disability and increase in function.

Hammer and Pfefer $^{50}$ treated a patient diagnosed with subacute lumbar compartment syndrome using GISTM. ${ }^{50}$ The patient after six treatments was asymptomatic, had nonrestrictive active and passive range of motions in all directions, normal functional tests and finally had a significant improvement in hamstring and right external rotators flexibility. Burke and colleagues ${ }^{52}$ conducted a pilot study on twentytwo patients with carpal tunnel syndrome using both the GISTM and ASTM techniques. There was an increase in nerve conduction latencies, increased wrist strength, increased range of motion and had greater patient satisfactory ratings. ${ }^{52}$ The present study also found an increase in ROM in all groups and movements according to percent change scores. Most particular is the GISTM/DBT group, all movements except EV was greater than the other two experimental groups.

Despite case reports and clinical studies, there have been no experimental studies conducted on GISTM technique and the effects on chronic ankle sprains. Most of the clinical studies and case reports that have been conducted have used animals and human subjects for lateral epicondylitis, ${ }^{40}$ patellar tendinopathy, ${ }^{49}$ rotator cuff tendinopathy ${ }^{36}$ and achilles tendinopathy. ${ }^{36,37,38,39,40,41}$ Only two studies involved ligaments. ${ }^{41,45}$ Melham did a case report on chronic ankle pain and fibrosis using the ASTM technique and found pain ceased with activity, the subjects range of motion improved, surgical scar matured, and the excessive fibrotic connective tissue around the ankle softened and diminished. ${ }^{41}$ Loghmani and colleagues ${ }^{45}$ conducted a study on rats using the GISTM technique in an attempt to examine the biomechanical and histological effects of instrumented-assisted 
cross fiber massage (IACFM) on early and long-term ligament healing. Twenty-one rats received nine treatments over a four-week period and thirty received thirty treatments over twelve weeks. At four and twelve weeks the ligaments were pulled to tensile failure. At four weeks a 31 percent increase in strength was found with 34 percent stiffer ligament structure compared to the untreated ligament. At twelve weeks a 15.4 percent stiffer ligament was found. Histological exams of the ligaments treated with IACFM were found to have greater fibroblast proliferation and more regular collagen alignment at either four or twelve weeks. ${ }^{45}$ In spite of this, no study has further evaluated the effects of chronic ankle instability and the condition of the ligaments after such an injury.

However, neither of these studies collected objective ROM data to help quantify the effectiveness of the GISTM treatment. The present study can objectively present there was an increase in ROM but cannot confirm the effectiveness of the GISTM treatment due to all groups increasing. However, the GISTM/DBT group did present with percent change scores for DF (26.2 percent), PF (26.3 percent), and INV (35.2 percent) compared to the $\mathrm{C} / \mathrm{DBT}$ and GISTM-S/DBT groups. It is understandable why EV would increase the least amongst all three groups as ROM for EV is between 5-10 degrees normally. ${ }^{1,17,109}$ However, a miniscule increase can provide a subject with considerably more functional movement than prior to participating in the study. Dynamic Postural Stability

The present study focused on between group differences for pre- and post-test results. Despite no group differences, there were improvements for all directions preand post-test. In addition, there were differences for the directions for A and PM, A and 
PL, and finally PM and PL. When observing percent change scores, the GISTM group had a greater percent change in the A and PM directions.

The actual test of the SEBT may be used to explain the differences. Many authors have identified specific excursions that are easier to perform than others. Specifically the posterior, posteromedial, and medial are usually identified as being the easiest to perform, whereas the anterior, anterolateral, and lateral have traditionally been the most difficult to perform..$^{77,78,79}$ In the study by Hertel et al. ${ }^{77}$, the lateral direction was by far the most difficult, and reach distances in the anterolateral direction were also significantly shorter than all of the other directions except lateral. Hertel et al. ${ }^{77}$ went further to say that the posterior and posteromedial directions seemed to yield the greatest reach distances, and were significantly longer than the other excursions. In the present study there was a significant increase in two of the three reach directions. This was noted that the most difficult reach direction was the PL, with the easier reaches the A, and PM.

Gribble et al. ${ }^{79}$ looked at the effects of fatigue and chronic ankle instability on dynamic postural control. They had all of the subjects complete five testing sessions in which sagittal-plane kinematics and reaching distances were recorded while they performed the anterior, medial and posterior excursions of the SEBT. They indicated that the involved side of the chronic ankle instability subjects had a significantly smaller reach distance and knee flexion angles for all three directions. They also noted that the fatiguing protocols decreases reach distance and knee flexion angles further. This is relevant to the present study because the involved side of the chronic ankle instability subjects in the GISTM/DBT group compared to the C/DBT and GISTM-S/DBT groups had a smaller increase in the direction for the PL excursion. The increase in the C/DBT 
and GISTM-S/DBT groups could be compared to the GSITM/DBT through the use of an injury model.

Using the DBT protocol, McKeon et al. ${ }^{35}$ found a significant group by time interaction for the PL and the PL reach components of the SEBT. In both directions, the balance-training group had greater reach distances in the post-test measures compared with the pre-test measures. ${ }^{35}$ However, the present study did not find any interactions. Moreover, the balance training group reached farther than the control group on the posttest measures but not on the pre-test measures. ${ }^{35}$ There were no significant changes in the Anterior reach direction between pre-test and post-test measures for either group. ${ }^{35}$ All three experimental groups performed the DBT program. According to percent change scores the GISTM/DBT group had higher percent changes in the A and PM directions. This is conflicting with the McKeon et al. ${ }^{35}$ study, however the present subject population was $17 \pm 7$ years old, whereas McKeon's et al. ${ }^{35}$ subjects were $22.2+4.5$ years old. The 4week progressive balancing-training program developed by McKeon et al. ${ }^{35}$ emphasized dynamic stabilization after a landing from a hop, in a variety of directions and conditions which significantly improved self-reported functional status, static postural control assessed by measures of time-to-boundary magnitude and variability, and dynamic postural control as assessed with SEBT reach distance.

Hale et al. ${ }^{34}$ in a 4 -week rehabilitation program found subjects with chronic ankle instability demonstrated deficits in postural control and SEBT reach tasks of the involved limb compared to the uninvolved limb and reported functional deficits of the involved limb compared to healthy subjects. Following rehabilitation, the chronic ankle instabilityrehabilitation group had greater SEBT reach improvements on the involved limb than the 
other groups and greater improvements in FADI and FADI-Sport scores. The two studies are similar in the fact that both were 4-week rehabilitation programs and involved injured subjects. However, the current study did not have healthy subjects to compare and contrast results. Also the rehabilitation programs between the two studies were incomparable due to Hale et al. ${ }^{34}$ using a more traditional rehabilitation program where the present study used the McKeon et al. ${ }^{35}$ approach to a more dynamic rehabilitation program.

The dynamic balance-training program is designed explicitly for an athlete, which is why improvement in functional mobility occurs. Athletes who suffer from functional ankle instability have decreased sensorimotor facilitation in the ankle as well as posture. The dynamic balance-training program works the body as a whole, increasing functional activity. The traditional measures used to assess the improvements in postural control may have lacked the sensitivity to detect improvements. Moreover, these balance-training programs may have not appropriately challenged the sensorimotor system to elicit a detectable change in postural control. ${ }^{35}$

From the dynamic systems perspective, the sensorimotor system has multiple degrees of freedom that afford a variety of strategies to be generated to maintain postural control. ${ }^{35}$ The constraints that act to limit these degrees of freedom include the complexity of the task and, the changes in adaptation. These constraints interact to shape postural control strategies to maintain an upright stance. ${ }^{35}$ However, CAI may place greater constraints on the sensorimotor system and may reduce the amount of degrees of freedom and, consequently, the amount of strategies available to maintain postural control. ${ }^{35}$ By purposely and progressively manipulating the task and the adaptation 
constraints on individuals with CAI, McKeon et $\mathrm{al}^{35}$ believed that the dynamic balancetraining program would aid the sensorimotor system in freeing up degrees of freedom that were not available to these individuals previously due to the constraints of CAI.

Therefore, a dynamic balance-training program that emphasizes the dynamic stabilization after perturbations such as predictable and unpredictable changes in direction, landing from a hop, and dynamic reaching tasks may prove more beneficial than the traditional balance training programs especially in those participating in sport due to improving the hip and ankle strategy. ${ }^{35}$ In the present study after the dynamic balance-training, all the individuals experienced a significant improvement in selfreported function, ROM, and dynamic postural control (SEBT), indicating that the sensorimotor system may no longer be deficient to the same magnitude. Ultrasonography

Data for ultrasonography was unavailable due to the variability of images for the two ligaments. Of the three pre- and post-test images that were available for visual comparison, no apparent differences could be visually analyzed for collagen alignment and neovascularization.

A normal Anterior Talofibular ligament (ATFL) is hyperechoic when the fibers are perpendicular to the ultrasound beam ${ }^{67,100,101,102,103,104}$ Being approximately two mm thick, during examination, the ATF ligament must be straight and tight from one insertion point to the other. ${ }^{67,100,101,102,103}$ The CFL forms the middle portion of the lateral collateral ligament and is between the inferior part of the lateral malleolus and the calcaneus, and runs in a slightly posterior oblique direction toward the calcaneus. ${ }^{67,100,101,102,103,104}$ The CFL is approximately two-three mm thick and is hyperechoic in the distal two-thirds only 
because of the obliquity of the proximal part. ${ }^{67,100,101,102,103,104}$ With sufficient knowledge of ankle technique, anatomy, and pathology, ultrasonography will be a reliable and effective means for detecting injury, especially that of ligamentous structures.

Clincial Implications and Limitations

Despite the non-significant differences between group or interactions for the outcomes, participants in the study showed beneficial improvements following the dynamic balance-training program and/or treatment. The use of the four-week GISTM and dynamic balance-training programs can increase ROM, sensorimotor facilitation, and stability for the ankle musculature, along with postural stability and endurance. Graston Instrumented-Assisted Soft Tissue Mobilization treatment is often used in combination with traditional and occasionally with core stabilization exercises in clinical settings. In the case in this study, GISTM was combined with a dynamic balance-training program, which challenged the sensorimotor system in multiple degrees of freedom to reduce the constraints limiting postural control. This particular combination with GISTM is more advantageous for athletes to allow for greater control of the degrees of freedom and constraints of sport. Various types of treatment and rehabilitation programs would also be used for a longer duration and given an appropriate rate of progression, one might expect to see further training/treatment adaptation. Furthermore, these training/treatment adaptations could be monitored using the tests employed in this study. The FAAM, FAAM Sport, VAS, ROM, and SEBT tests were easy to administer and required no specialized equipment/facilities. The ultrasonography, on the other hand was challenging due to the inherent learning curve associated with using the technique and that it is very operator dependent. Despite the ease and simplicity of administration of the FAAM, 
FAAM Sport, VAS, ROM, and SEBT, these tests are highly reliable and valid indicators of performance that can be used for both research and clinical purposes. Even though statistical significance wasn't apparent due to the small sample size, clinically the GISTM/DBT group exhibited greater improvement in percent change scores with the FAAM Sport, VAS, three movements in ROM (DF, PF, INV), and two directions in SEBT (A, PM) than the other two experimental groups.

The primary limitations of the present study included a small sample size, limited generalizability of results, use of a relatively brief dynamic balance-training program, and ultrasonography. This study used a sample of convenience at a North Central high school and Mid-Atlantic University of only injured physically active individuals. While the sample was of convenience, scheduling sessions was challenging due to snow days and bad-road conditions which disabled the subjects from making their scheduled time. Given the small sample size, the results cannot be generalized to a broader population. If used with other participants, treatment and dynamic balance-training program adaptations might vary depending on a number of additional factors that contribute to individual variability including previous injury and rehabilitation history and current fitness level. While the length of the employed GISTM treatment and dynamic balance-training program was similar to that used in previous studies, a longer period of time might be necessary to demonstrate differences among various groups. Unfortunately, there is no research suggesting the optimal number of treatment sessions to perform in a GISTM treatment and dynamic balance-training program. 


\section{CONCLUSIONS}

There was a significant difference with test for FAAM, FAAM Sport, VAS, ROM, and SEBT with post-test results greater except for VAS, which decreased. There were also significant differences for movement (ROM) and direction (SEBT). While there were no significant differences between experimental groups, an increase was evident in all pre-test to post-test results for all groups with the GISTM/DBT group showing the best percent change improvements followed by the C/DBT and GISTMS/DBT groups. The four-week GISTM and dynamic balance-training program could be used to increase ROM, sensorimotor facilitation, and stability for the ankle musculature, along with postural stability and endurance in all athletes suffering from chronic ankle instability. These results support the combined use of GISTM and rehabilitation exercises in clinical practice. 


\section{REFERENCES}

1. Hertel J. Functional anatomy, pathomechanics and pathophysiology of lateral instability. J Athl Train. 2002;37(4):364-375.

2. Kumai T, Takakura Y, Rufai A, Milz S, Benjamin M. The functional anatomy of the human anterior talofibular ligament in relation to ankle sprains. $J$ Anat. 2002;200:457-465.

3. Hopkins JT, Palmieri R. Effects of ankle joint effusion on lower leg function. Clin J Sport Med. 2004;14(1):1-7.

4. Lynch SA. Assessment of the injured ankle in the athlete. $J$ Athl Train. 2002;37(4):406-412.

5. Miller CA, Bosco JA III. Lateral ankle and subtalar instability. Bull Hosp Jt. Dis. 2001-2002;60(3-4):143-149.

6. Hintermann, B. Biomechanics of the unstable ankle joint and clinical implications. Med Sci Sport Exerc. 1999;31(7):S459-S469.

7. Safran MR, Benedetti RS, Bartolozzi AR III, Mandelbaum BR. Lateral ankle sprains: a comprehensive review Part 1: etiology, pathoanatomy, histopathogenesis, and diagnosis. Med Sci Sport Exerc. 1999;31(7):S429-S437.

8. Pollard H, Sim P, McHardy A. Lateral ankle injury: literature review and report of two cases. ACO. 2002;10(1):21-30.

9. Taser F, Shafiq Q, Ebraheim NA. Anatomy of lateral ankle ligaments and their relationship to bony landmarks. Surg Radiol Anat. 2006;28:391-397.

10. Hubbard TJ, Kaminski TW. Kinesthesia is not affected by functional ankle instability status. J Athl Train. 2002;37(4):481-486.

11. Morrison KE, Kaminski TW. Foot characteristics in association with inversion ankle injury. J Athl Train. 2007;42(1):135-142.

12. Halasi T, Kynsburg A, Tallay A, Berkes I. Changes in joint position sense after surgically treated chronic lateral ankle instability. Br J Sports Med. 2005;39:818-824.

13. Docherty CL, Moore JH, Arnold BL. Effects of strength training on strength development and joint position sense in functionally unstable ankles. $J$ Athl Train. 1998;33(4):310-314. 
14. Kynsburg A, Halasi T, Tallay A, Berkes I. Changes in joint position sense after conservatively treated chronic lateral ankle instability. Knee Surg Sport $\operatorname{Tr}$ A. 2006;14:1299-1306.

15. Hertel J, Denegar, CR, Monroe MM, Stokes WL. Talocrural and subtalar joint instability after lateral ankle sprain. Med Sci Sport Exerc. 1999;31(11):1501-1508.

16. Wright IC, Neptune RR, Van Den Bogert AJ, Nigg BM. The influence of foot positioning on ankle sprains. $J$ Biomech. 2000;33:513-519.

17. Hubbard TJ, Hertel J. Mechanical contributions to chronic lateral ankle instability. Sports Med. 2006;36(3):263-277.

18. Hertel J. Functional instability following lateral ankle sprains. Sports Med. 2000;29(5)361-371.

19. Kaminski TW, Perrin DH, Gansneder BM. Eversion strength analysis of uninjured and functionally unstable ankles. J Athl Train. 1999.34(3):239-245.

20. Hubbard TJ, Kramer LC, Denegar CR, Hertel J. Correlations among multiple measures of functional and mechanical instability in subjects with chronic ankle instability. J Athl Train. 2007;42(3):361-366.

21. Willems T, Witvrouw E, Verstuyft J, Vaes P, De Clercq D. Proprioception and muscle strength in subjects with a history of ankle sprains and chronic instability. $J$ Athl Train. 2002;37(4):487-493.

22. Hertel J, Denegar CR, Monroe MM, Stokes WL. Talocrural and subtalar joint instability after lateral ankle sprain. Med Sci Sports Exerc. 1999;31(11):1501-1508.

23. Docherty CL, Moore JH, Arnold BL. Effects of Strength Training on Strength Development and Joint Position Sense in Functionally Unstable Ankles. J Athl Train. 1998;33(4):310-314.

24. Sharma P, Maffulli N. Biology of tendon injury: healing, modeling and remodeling. $J$ Musculoskelet Neuronal Interact. 2006;6(2):181-190.

25. Houglum PA. Soft Tissue healing and its impact on rehabilitation. JSR. 1992;1:19-39.

26. Butterfield TA, Best TM, Merrick MA. The dual roles of neutrophils and macrophages in inflammation: a critical balance between tissue damage and repair. $J$ Athl Train. 2006;41(4):457-465.

27. Clement D. The Science and Theory of Rehabilitation. ATTR 625. Class notes. West Virginia University. College of Physical Activity and Sport Sciences. 
28. Kaminski TW, Perrin DH, Gansneder BM. Eversion strength analysis of uninjured and functionally unstable ankles. J Athl Train. 1999;34(3):239-245.

29. Kaminski TW, Hartsell HD. Factors contributing to chronic ankle instability: a strength perspective. J Athl Train. 2002;37(4):394-405.

30. Hubbard TJ, Kramer LC, Denegar CR, Hertel J. Contributing factors to chronic ankle instability. Foot Ankle Int. 2007;28(3):343-54.

31. Hertel J. Sensorimotor deficits with ankle sprains and chronic ankle instability. Clin Sports Med. 2008;27:353-370.

32. Lephart SM, Pincivero DM, Giraldo JL, Fu FH. The role of proprioception in the management and rehabilitation of athletic injuries. Am J Sports Med. 1997;25(1):130137.

33. Bernier JN, Perrin Dh. Effects of coordination training on proprioception of the functionally unstable ankle. J Orthop Sports Phys Ther. 1998;27(4):264-275.

34. Hertel J, Hale SA, Olmsted LC. The effect of a 4-week comprehensive rehabilitation program on postural control and lower extremity function in individuals with chronic ankle instability. J Orthop Sports Phys Ther. 2007;37(6):303-11.

35. McKeon PO, Ingersoll CD, Kerrigan DC, Saliba E, Bennett B, Hertel J. Balance training improves function and postural control in those with chronic ankle instability. Med Sci Sport Exerc. 2008;40(10):1810-1819.

36. Davidson CJ, Ganion LR, Gehlsen GM, Verhoestra B, Roepke JE, Sevier TL. Rat tendon morphologic and functional changes resulting from soft tissue mobilization. Med Sci Sport Exerc. 1997;29(3):313-319.

37. Gehlsen GM, Ganion LR, Helfst R. Fibroblast responses to variation in soft tissue mobilization pressure. Med Sci Sport Exerc. 1999;31(4):531-535.

38. Howitt S, Wong J, Zabukovec S. The conservative treatment of trigger thumb using graston techniques and active release techniques. J Can Chiropr Assoc. 2006;50(4):249-254.

39. Sevier TL, Wilson JK. Treating lateral epicondylitis. Sports Med. 1999;28(5):375380.

40. Sevier TL, Helfst RH, Stover SA, Wilson JK. Clinical trends on tendinitis. Work. 2000;14:123-126.

41. Melham, TJ, Sevier TL, Malnofski MJ, Wilson JK, Robert HJ. Chronic ankle pain and fibrosis successfully treated with a new noninvasive augmented soft tissue 
mobilization technique (ASTM): a case report. Med Sci Sport Exerc. 1998;30(6):801804.

42. Safran MR, Zachazewski JE, nenedetti RS, Bartolozzi AR III, Mandelbaum R. Lateral ankle sprains: a comprehensive review part 2: treatment and rehabilitation with an emphasis on the athlete. Med Sci Sport Exerc. 1999;31(7):S438-S447.

43. Miller CA, Bosco JA. Lateral ankle and subtalar instability. Bull Hosp Jt Dis . 20012002;60(3-4):143-9.

44. Karlsson J, Brandsson S, Jerre R. Ligament injuries of the ankle joint. Curr Opin Orthop. 1999;10:179-185.

45. Loghmani T, Burr DB, Warden SJ. Biomechanical and histological effects of instrument-assisted cross fiber massage on acute and long-term ligament healing. Med Sci Sport Exerc. 2008.

46. Delahunt E, Monaghan K, Caulfield B. Altered neuromuscular control and ankle joint kinematics during walking in subjects with functional instability of the ankle joint. Am J Sports Med. 2006;34(12):1970-1976.

47. Young CC, Niedfeldt MW, Morris GA, Eerkes KJ. Clinical examination of the foot and ankle. Prim Care Clin Office Pract. 2005;32:105-132.

48. Coughlan G, Caulfield B. A 4-week neuromuscular training program and gait patterns at the ankle joint. $J$ Athl Train. 2007;42(1):51-59.

49. Wilson JK, Sevier TL, Helfst R, Honing EW, Thomann A. Comparison of rehabilitation methods in the treatment of patellar tendinitis. J Sport Rehabil. 2000;9:304-314.

50. Hammer WI, Pfefer MT. Treatment of a case of subacute lumbar compartment syndrome using the graston technique. J Manipulative Physio Ther. 2005;28:199-204.

51. Aspegren D, Hyde T, Miller M. Conservative treatment of a female collegiate volleyball player with costochondritis. J Manipulative Physiol Ther. 2007;30:321325.

52. Burke J, Buchberger DJ, Carey-Loghmani MT, Dougherty PE, Greco DS, Dishman JD. A pilot study comparing two manual therapy interventions for carpal tunnel syndrome. J Manipulative Physiol Ther. 2007;30:50-61.

53. Hubbard TJ, Kaminski TW, Vander Griend RA, Kovaleski JE. Quantitative assessment of mechanical laxity in the functionally unstable ankle. Med Sci Sport Exerc. 2004;36(5):760-766. 
54. Docherty CL, Gansneder BM, Arnold BL, Hurwitz SR. Development and reliability of the ankle instability instrument. J Athl Train. 2006;41(2):152-158.

55. Hale SA, Hertel J. Reliability and sensitivity of the foot and ankle disability index in subjects with chronic ankle instability. J Athl Train. 2005;40(1):35-40.

56. Martin RL et al. Evidence of validity for the Foot and Ankle Ability Measure (FAAM). Foot Ankle Int. 2005;26(11):968-983.

57. Carcia CR, Martin RL, Drouin JM. Validity of the Foot and Ankle Ability Measure in athletes with chronic ankle instability. J Athl Train. 2008;43(2):179-183.

58. Choiniere M, Amsel R. A visual analogue thermometer for measuring pain intensity. J Pain Symptom Manage. 1996;11(5):299-311.

59. Kahl C, Cleland JA. Visual analogue scale, numeric pain rating scale and the McGill pain questionnaire: an overview of psychometric properties. Phys Ther Rev. 2005;10(2):123-128.

60. Herr K, Spratt KF, Garand L. Evaluation of the Iowa pain thermometer and other selected pain intensity scales in younger and older adults cohorts using controlled clinical pain: a preliminary study. Pain Med. 2007;8(7):585-600.

61. Myles PS, Troedel S, Boquest M, Reeves M. The pain visual analog scale: is it linear or nonlinear. Anesth Analg. 1999;89:1517-1520.

62. Gloth FM III, Scheve AA, Stober CV, Chow S, Prosser J. The functional pain scale: reliability, validity, and responsiveness in an elderly population. J Am Med Dir Assoc. 2001;2:110-114.

63. Menadue C, Raymond J, Kilbreath SL, Refshauge KM, Adams R. Reliability of two goniometric methods of measuring active inversion and eversion range of motion at the ankle. BMC Musculoskelet Disord. 2006;28(7):60.

64. Rome K, Cowieson F. A reliability study of the universal goniometer, fluid goniometer, and electrogoniometer for the measurement of ankle dorsiflexion. Foot Ankle Int. 1996;17(1):28-32.

65. Youdas JW, Bogard CL, Suman VJ. Reliability of goniometric measurements and visual estimates of ankle joint active range of motion obtained in a clinical setting. Arch Phys Med Rehabil. 1993;74(10):1113-8.

66. Bovens AM, van Baak MA, Vrencken JG, Wijnen JA, Verstappen FT. Variability and reliability of joint measurements. Am J Sports Med. 1990;18(1):58-63. 
67. Finlay K, Friedman L. Ultrasonography of the lower extremity. Orthop Clin N Am. 2006;37:245-275.

68. van Dijk C, Mol B, Lim L, Marti R, Bossuyt R. Diagnosis of ligament rupture of the ankle joint. Acta Orthop Scand. 1996;67(6):566-570.

69. Earl JE, Hertel J. Lower-extremity muscle activation during the star excursion balance tests. J Sport Rehabil. 2001;10(2):93-104.

70. Gribble PA, Hertel J. Considerations for normalizing measures of the star excursion balance test. Measure Phys Ed Exer Sci. 2003;7(2):89-100.

71. Gribble P, Kaminski TW. Research digest. The star excursion balance test as a measurement tool. Athl Ther Today. 2003;8(2):46-47.

72. Kinzey SJ. The reliability of the star-excursion test in assessing dynamic balance. $J$ Orthop Sports Phys Ther. 1998;27(5):356-360.

73. Olmsted LC, Carcia CR, Hertel J, Shultz SJ. Efficacy of the Star Excursion Balance Tests in detecting reach deficits in subjects with chronic ankle instability. $J$ Athl Train. 2002;37(4):501-506.

74. Gribble P, Hertel J, Denegar C, Buckley W. Reliability and validity of a 2-D video digitizing system during a static and a dynamic task. J Sport Rehabil. 2005;14:137149.

75. Cote KP, Brunet ME, Gansneder BM, Shultz SJ. Effects of pronated and supinated foot postures on static and dynamic postural stability. J Athl Train. 2005;40(1):41-46.

76. Hertel J, Braham RA, Hale SA, Olmsted LC. Simplifying the star excursion balance test: factors analyses of subjects with and without ankle instability. $J$ Orthop Sports Phys Ther. 2006;36(3):131-7.

77. Nakagawa L. Performance in static, dynamic, and clinical tests of postural control in individuals with recurrent ankle sprains. $J$ Sport Rehabil. 2004;13(3):255-268.

78. Hertel J, Denegar CR, Buckley WE, Sharkey NA, Stokes WL. Effect of rear-foot orthotics on postural control in healthy subjects. J Sport Rehabil. 2001;10:36-47.

79. Gribble PA, Hertel J, Denegar CR, Buckley WE. The effects of fatigue and chronic ankle instability on dynamic postural control. J Athl Train. 2004;39(4):321-329.

80. Olmsted-Kramer LC, Hertel J. Preventing recurrent lateral ankle sprains: an evidencebased approach. Athl Ther Today. 2004;9(6):19-22,34-35,68. 
81. Olmsted LC, Carcia CR, Hertel J, Shultz SJ. Efficacy of the star excursion balance tests in detecting reach deficits in subjects with chronic ankle instability. J Athl Train. 2002;37(4):501-506.

82. Hartzell D. (n.d). Flex Band Flexibility Routine for all sports. In Flex Band by Jump Stretch. Retrieved. 2008 Sept. 1, from Website: http://www.flexbandsforsports.com/flexibilityroutine.html.

83. Rozzi SL et al. Balance training for persons with functionally unstable ankles. $J$ Orthop Sports Phys Ther. 1999;29(8):478-486. 
APPENDICES 


\section{APPENDIX A}

\section{THE PROBLEM}

The Research Question

The prevalence of ankle sprains varies between $75-85$ percent $^{4,6,9,10,13,14,15}$ of all ankle injuries, with approximately 85 percent of these sprains caused by an inversion trauma. ${ }^{4,6,9,10,13,14,15}$ Twenty to 40 percent $^{6,7}$ of these patients will go on to experience chronic instability and subsequent disability. However, there is conflicting statistics on the percentage of ankle sprains and those caused by inversion trauma. With better understanding of the function of the ligament structures and biomechanics of the ankle, subsequent injury may be preventable. , $5,6,7,9,10,13,14,15,16,21^{-1}$

It is postulated that the repair process is similar in function with chronic injuries to that of acute injuries. When a ligamentous soft tissue injury occurs, one of the damaging mechanisms is scar tissue formation. To assist in removal of excessive scar tissue and the healing process, a type of soft tissue mobilization must be used. Augmented Soft Tissue Mobilization (ASTM) introduces a more controlled amount of microtrauma into an area of excessive scar tissue, therefore, the response of the tendon to this microtrauma could involve augmentation of the healing process..$^{36,37,38,39,40,41}$ Graston Instrumented-Assisted Soft Tissue Mobilization (GISTM), another variation of this technique, also has shown that it does increase the fibroblast response with the controlled

movement of the instruments. ${ }^{2,5,6,9,37,42,44}$ Although most of the original research has been on tendons using animal and human subjects, there have been two studies reported using $\operatorname{ASTM}^{41}$ and GISTM ${ }^{45}$ on ligaments. 
Despite the lack of presence of inflammatory cells, healing is evident following GISTM treatment using an animal model. ${ }^{36,37,38,39,40,41}$ In one study, a significant increase in fibroblast number was noted in two of the experimental groups, which illustrates one of the reported features of tendon healing: proliferation of fibroblasts. ${ }^{36}$ Gait analysis studies, reported evident healing and earlier recovery of limb function following a collagenase injury to the achilles tendon. ${ }^{6,15,16,46,47,48}$ Early restoration of function, promotes mechanical forces to act more rapidly to further collagen fiber realignment and thus accelerate the completion of tendon repair. ${ }^{2,5,6,7,9,37,44}$

Five clinical trial studies, over the past eight years, have been conducted using the ASTM or GISTM technique. Sevier and colleagues ${ }^{40}$ conducted a trial of ASTM on a patient suffering for two years from chronic lateral epicondylitis. ${ }^{40}$ The patient's pain and disability significantly decreased with an increase in strength, and discontinued use of the tennis elbow brace. Wilson and colleagues ${ }^{49}$ performed ASTM on twenty patients with patellar tendinitis. Sixty percent of the traditional rehabilitation group symptoms and pain resolved with 100 percent of the ASTM group experiencing complete resolution of symptoms and pain. The remaining 40 percent of the patients from the traditional rehabilitation group, crossed-over to the ASTM group with 50 percent undergoing resolution of symptoms and pain. Hammer and $\mathrm{Pfefer}^{50}$ treated a patient diagnosed with subacute lumbar compartment syndrome using GISTM. After several treatments the patient was asymptomatic, had nonrestrictive active and passive range of motions in all directions, normal functional tests and a significant improvement in hamstring and right external rotator flexibility. Aspegren and colleagues ${ }^{51}$ used GISTM on a patient suffering from costochondritis. From the initial visit, there was a decrease in pain and a significant 
improvement in the Dallas Pain Questionnaire. ${ }^{51}$ Lastly, Burke and colleagues ${ }^{52}$

conducted a pilot study on twenty-two patients with carpal tunnel syndrome using both the GISTM and ASTM techniques. There was an increase in nerve conduction latencies, increased wrist strength, increased range of motion and had greater patient satisfactory ratings.

Despite case reports and clinical studies, there have been no experimental studies conducted on GISTM technique and the effects on chronic ankle sprains. Most of the clinical studies and case reports that have been conducted have used animals and human subjects for lateral epicondylitis, ${ }^{40}$ patellar tendinopathy, ${ }^{49}$ rotator cuff tendinopathy ${ }^{36}$ and achilles tendinopathy. ${ }^{36,37,38,39,40,41}$ Only two studies involved ligaments. ${ }^{41,45}$ Melham did a case report on chronic ankle pain and fibrosis using the ASTM technique and found pain ceased with activity, the subjects range of motion improved, surgical scar matured, and the excessive fibrotic connective tissue around the ankle softened and diminished. ${ }^{41}$ Loghmani and colleagues ${ }^{45}$ conducted a study on rats using the GISTM technique in an attempt to examine the biomechanical and histological effects of instrumented-assisted cross fiber massage (IACFM) on early and long-term ligament healing. Twenty-one rats received nine treatments over a four-week period and thirty received thirty treatments over twelve weeks. At four and twelve weeks the ligaments were pulled to tensile failure. At four weeks a 31 percent increase in strength was found with 34 percent stiffer ligament structure compared to the untreated ligament. At twelve weeks a 15.4 percent stiffer ligament was found. Histological exams of the ligaments treated with IACFM were found to have greater fibroblast proliferation and more regular collagen alignment at 
either four or twelve weeks. ${ }^{45}$ In spite of this, no study has furthered evaluated the effects of chronic ankle instability and the condition of the ligaments after such an injury.

Beneficial effects, however, have been noted with dynamic postural balance over a four-week period ${ }^{34,35}$ using chronic ankle instability subjects. Since only a case report exists in the literature for chronic ankle instability using Graston Instrument-Assisted Soft Tissue Mobilization, the intent of this study is to further evaluate the effects of Graston Instrumented-Assisted Soft Tissue Mobilization and dynamic postural balance on improvements in healing and function. Therefore, the present study aims to investigate the affects of Graston Instrumented-Assisted Soft Tissue Mobilization (GISTM) technique and its influence on improving range of motion, neovascularization, collagen alignment, pain and disability in individuals experiencing chronic ankle instability. Due to no studies exploring the combination of both GISTM treatment and rehabilitation protocol being used.

Therefore, the following research question is being proposed: Can the Graston Instrumented-Assisted Soft Tissue Mobilization (GISTM) technique improve range of motion, neovascularization, collagen alignment, pain and disability in individuals experiencing chronic ankle sprains?

Experimental Hypotheses

1. There will be a greater increase in the talocrural range of motion in the group using the Graston Instrumented-Assisted Soft Tissue Mobilization technique group.

2. There will be a greater increase in neovascularization in the Graston Instrumented-Assisted Soft Tissue Mobilization technique group.

3. There will be a significant difference in collagen alignment in the Graston Instrumented-Assisted Soft Tissue Mobilization group. 
4. There will be a decrease in the self-report FADI, FADI Sport and VAS questionnaires in the Graston Instrumented-Assisted Soft Tissue Mobilization technique group.

5. There will be a significant difference in pre- and post-test results in the Graston Instrumented-Assisted Soft Tissue Mobilization group compared to the traditional rehabilitation group and the traditional rehabilitation group plus the sham Graston Instrumented-Assisted Soft Tissue Mobilization.

Assumptions

1. All participants in this study will meet all of the inclusion criteria while no participant shall be incorporated if meeting any of the exclusion criteria.

2. Participants will give maximum effort and perform to the best of their ability during training periods and pre/post testing.

3. The participants will be compliant with their designated rehabilitative programs and will return for post-testing.

4. The clinician will be proficient in performing the Graston Instrumented-Assisted Soft Tissue Mobilization technique.

\section{Delimitations}

1. The small sample size will not be generalizable to the population as a whole.

2. Chronic ankle sprains at one institution will not be generalizable to the population as a whole.

3. The results of the study will only be generalizable to the use of the Graston Instrumented-Assisted Soft Tissue Mobilization technique.

Operational Definitions

1. Ankle Arthrometer - Device used to measure mechanical instability. ${ }^{53}$

2. Anterior Talofibular Ligament - A flat, weak band that extends anteriorly and medially from the lateral malleolus to the neck of the talus. ${ }^{6,7,15}$

3. Athlete - Someone with athletic ability. ${ }^{83}$

4. Brushing - Superficial, linear stroking motions: small amplitude; any direction. ${ }^{99}$

5. Calcanealofibular Ligament - A round cord that passes posteriorly and inferiorly from the tip of the lateral malleolus to the lateral surface of the calcaneus. ${ }^{6,7,15}$ 
6. Chronic Ankle Sprain - An injury to the lateral ankle ligaments that has occurred more than once. ${ }^{7,18}$

7. Collagen - The collagen arrangement in ligamentous tissue that is comprised of structural, fibrous protein found in all connective tissues. ${ }^{84}$

8. Color Doppler Ultrasonography - Diagnostic imagining using the doppler effect to identify vascular abnormalities in structures. ${ }^{67}$

9. Contralateral - Referring to the opposite side of the body. ${ }^{47}$

10. Disability Index - Assesses functional limitations related to foot and ankle conditions. $^{55}$

11. Dominant Leg - Preferred leg used when performing tasks such as kicking a soccer ball. ${ }^{47}$

12. Dynamic Balance-Training - Emphasis on the dynamic stabilization after perturbations such as predictable and unpredictable changes in direction, landing, from a hop, and dynamic reaching. ${ }^{35}$

13. Dynamic Postural Control - The ability to stabilize oneself while stationary or during movement on a flat or odd surface. ${ }^{47}$

14. Fanning - A fanning stroke is characterized by the instrument contact points moving at different rates in an anchored path. One end of the instrument is stabilized, serving as the fulcrum of motion, while the other end is moving, with the resistance at the end of the instrument that is moving. ${ }^{99}$

15. Functional Instability -Recurrent joint instability and the sensation of joint instability due to the contributions of any neuromuscular deficit. ${ }^{18}$

16. Graston Instrumented-Assisted Soft Tissue Mobilization (GISTM) Technique An advanced form of soft tissue mobilization combined with targeted stretching and strengthening exercises that is used to detect and release scar tissue, adhesions, and fascial restrictions. ${ }^{38}$

17. Joint Position Sense - The body's conscious awareness of joint position and movement resulting from the proprioceptive input to the central nervous system. ${ }^{14}$

18. J-Stroke - Superficial or deep stroke in a J-shaped pattern. ${ }^{99}$

19. Lateral Ankle Injury - Any harm caused to the distal fibula, tarsal bones, metatarsal bones, or ligaments, muscles, or tendons surrounding these bones when 
the ankle is placed in extremes of physiological ROM. ${ }^{6,7,15}$

20. Mechanical Instability - Laxity of the joint due to structural damage to ligamentous tissues which support the joint. ${ }^{18}$

21. Neovascularization - The proliferation of new blood vessels, especially in tissues where circulation has been impaired by trauma. ${ }^{86}$

22. Posterior Talofibular Ligament - A thick, fairly strong band that runs horizontally medially and slightly posteriorly from the malleolar fossa to the lateral tubercle of the talus. ${ }^{6,7,15}$

23. Proprioception -The specialized variation of the sensory modality of touch that encompasses the sensation of movement (kinesthesia) and joint position (joint position sense). ${ }^{21}$

24. Range of Motion - The degrees a limb can be moved through a full motion. ${ }^{47}$

25. Rehabilitation - Exercises and modalities used in order to help promote healing and return a person with an injury to pre-injury state. ${ }^{87}$

26. Scar Tissue - A dense, fibrous connective tissue that joins the torn fibers of a ligament of tendon. ${ }^{41}$

27. Scooping - Superficial or deep stroke in a scooping pattern. ${ }^{99}$

28. Subtalar Joint (STJ) - Sits inferiorly to the talocrural joint. The talus is the ceiling of the subtalar joint while the calcaneus is the floor. The STJ allows the foot to move into inversion and eversion. $5,11,47,88,89$

29. Sweeping/Scanning - A sweeping stroke is characterized by the instrument contact points moving in one direction at the same rate in either a linear or curvilinear path. $^{99}$

30. Talocrural Joint (TCJ) -The tibia forms the medial portion of the ankle; the fibula forms the lateral portions of the ankle; and the talus forms the floor of the inferior portion of the TCJ. The TCJ is responsible for dorsiflexion and plantarflexion motions of the foot. ${ }^{5,11,47,88,89}$

31. Treatment - Physically treating an injury with modalities and rehabilitation. ${ }^{90}$

32. Treatment time - The amount of time it takes to apply treatment. ${ }^{90}$

33. Ultrasonography - Diagnostic imaging in which ultrasound is used to image internal body structures. $^{67}$ 
34. Unilateral - Referring to one side of the body only (a unilateral stance is standing on one leg only). ${ }^{47}$

Limitations

1. Participants will drop out of the study at any time or not return for post-testing due to injury or other reasons.

2. Participants will not be compliant with the rehabilitation programs.

3. Participants will be exposed to unexpected events that occur throughout the course of the study.

4. Participants will maturate during the study.

5. Participants will be subjected to selection bias.

Significance of the Study

To the author's knowledge, this will be the first study using the Graston Instrumented-Assisted Soft Tissue Mobilization (GISTM) technique on humans along with a dynamic balance-training program in relationship to chronic ankle sprains in a rehabilitation program. Based on past research GISTM increased range of motion, increased neovascularization, realigned collagen, remodeled scar tissue, and decreased pain and disability. Therefore, since chronic ankle sprains are caused by functional and mechanical instability related to decreased range of motion and the healing process within and surrounding the ankle, use of GISTM will demonstrate the same benefits. If increased range of motion, collagen realignment and restructive scar tissue is remodeled in four to eight sessions, then the next step in the protocol would be to incorporate GISTM as an accessory treatment for a chronic ankle instability.

This study will bring new knowledge to ATC's as well as other allied health professionals and those that practice sports medicine. If this study is able to determine, GISTM as an adjunct treatment in the rehabilitation protocol being more effective than a 
dynamic balance-training program alone, then ATC's will be able to incorporate additional rehabilitative measures for treatment of lateral ankle sprains. With an improved, better-quality rehabilitation protocol comes fewer occurrences of reinjury and days lost in participation. In an age where technology is advancing and in a setting where rehabilitative techniques encompass new styles and techniques, ATC's could benefit from having knowledge of a simple technique that will aid in the healing process for the injured individual and possibly return an injured ligament structure back to pre-injury status.

Finally, to build upon the current knowledge base that exists in sports medicine in rehabilitating chronic ankles sprains, this material needs to be disseminated to ATC's and other allied health professionals. A broader knowledge on GISTM and chronic ankle sprains can be found in journal publications, presentations, workshops and CEU courses, both locally and at national meetings. Certified athletic trainers will be able to more effectively treat chronic ankle injuries with an additional therapeutic technique along with the dynamic balance-training program used. 


\section{APPENDIX B}

\section{LITERATURE REVIEW}

Introduction

Chronic ankle instability is a common problem after acute lateral ankle sprains. Whether instability is the result of injury with or without demonstrable ankle ligament laxity is unclear. ${ }^{1,2,3,4,9,10,12,13,14}$ The musculature, which in part leads to the instability along with the ligaments, can be separated into the talocrural and subtalar joints. The talocrural joint consists of the muscles of the anterior compartment and posterior compartment and the superficial ligaments of the lateral side along with the deltoid ligament medially. ${ }^{1,5,6,9,15,91}$ The subtalar joint consists of the muscles of the lateral and deep posterior compartments and the deep ligaments of the lateral side along with the retinacula. ${ }^{1,5,6,9,15,91}$ During dorsiflexion and plantarflexion the muscles and ligaments of the talocrural joint are constantly being eccentrically and concentrically contracted. ${ }^{92,93}$ The same can be said for the muscles and ligaments of the subtalar joint that provide inversion and eversion to the ankle joint complex. ${ }^{92,93}$ The occurrence of ankle sprains is very common throughout sports and is the single most injury leading to time loss from participation. $^{7}$ The most frequent cause of lateral ankle sprains comes from an excessive supinated-inverted position of the ankle. ${ }^{1,7,11,16,89,94}$ Several subjective tests exist that provide a differential diagnosis of the severity of the injury. ${ }^{4,95}$ However, the reliability of objective measures is also applicable and essential., ${ }^{4,95}$

Once injury, not necessarily severity of injury has been determined the

rehabilitation process begins immediately. ${ }^{96,97}$ A single protocol does not exist for ankle rehabilitation partly due to the individualization of each program specifically for the 
injured athlete. ${ }^{96,97}$ In four studies, ${ }^{40,41,49,52}$ a five to ten-minute dynamic warm-up was completed to activate and warm the muscles, followed by the Graston InstrumentAssisted Soft Tissue Mobilization treatment, and concluded with a dynamic strengthening program and cryotherapy. A new technique called Graston InstrumentedAssisted Soft Tissue Mobilization is now being utilized as an adjunct treatment with acute and chronic injuries. ${ }^{36,37,38,40,41,49,50,51,52,98}$ The GISTM increases range of motion, increases neovascularization, collagen alignment, scar tissue remodeling and decreases pain and disability. ${ }^{36,37,38,40,41,49,50,51,52,82,98}$ With the GISTM technique being incorporated into the rehabilitation protocol, definitive measures of objective data demonstrating the GISTM technique's objectives success, need to be reported. ${ }^{36,37,38,40,41,49,50,51,52,98}$ Ultrasonography is a widely used tool and has been proven to be reliable in diagnosising ankle sprains and their severity. ${ }^{67,100,101,102,103,104}$ Objective data is imperative for repeatability of a study, however, having subjective data from the patient's perspective is also beneficial. ${ }^{55,58,59,60,105,106,107}$

In the literature review, the anatomy of the ankle, biomechanics of the ankle joint, epidemiology, etiology, assessment tools for the ankle, the components of traditional rehabilitation, Graston Instrumented-Assisted Soft Tissue Mobilization technique, ultrasonography, the visual analogue scale (VAS) of Pain and finally the foot and ankle disability index will be discussed.

Anatomy

The functional anatomy of the ankle complex is intricate and imperative to know when treating ankle sprains. The ankle complex is comprised of three articulations: the talocrural joint, the subtalar joint, and the distal tibiofibular syndesmosis. ${ }^{1,5}$ These joints 
allow for movement of the rearfoot. Movement of the rearfoot can occur in the sagittal, frontal, and the transverse-planes. ${ }^{1,5}$ In the case of rearfoot movement, a lateral ankle sprain can occur affecting the talocrural and subtalar joints.

Talocrural joint: The talocrural joint is a hinge joint allowing dorsiflexion and plantarflexion movements. The joint is formed by the articulation of the dome of the talus, the medial malleolus, the tibial plafond, and the lateral malleolus. ${ }^{1,5,69.15}$ The shape allows for torque of the leg to be transmitted into the foot. ${ }^{1,5,69.15}$ The talocrural joint receives ligamentous support from a joint capsule and several ligaments, including the anterior talofibular ligament (ATFL), posterior talofibular ligament (PTFL), calcaneofibular ligament (CFL), and deltoid ligament. ${ }^{1,5,6,9.15}$ Lateral support is provided by the ATFL, PTFL, and CFL, while the deltoid ligament provides medial. The anatomical locations, origin, insertion, angle and length/width of the ligaments are described in more detail in Table B1. When the ankle complex is fully loaded, the articular surfaces are the primary stabilizers against excessive talar rotation and translation; however, the contribution of the ligaments to talocrural joint stability is crucial. ${ }^{1,5,69.15}$ The strain of the ATFL increases as the ankle moves from dorsiflexion into plantarflexion. ${ }^{1,5,69.15}$ The ATFL demonstrates lower maximal load and energy to failure values under tensile stress as compared with the PTFL, CFL, and deltoid ligament. ${ }^{1,5,6,9.15}$ The broader insertion of the PTFL on both the talus and fibula, provides restraint to both inversion and internal rotation of the loaded talocrural joint. ${ }^{1,5,69.15}$ The CFL restricts excessive supination of both the talocrural and subtalar joints. ${ }^{1,5,6,9.15}$ 
Table B1. Anatomical Location of Talocrural Joint Ligaments. ${ }^{1,5,6,9.15}$

\begin{tabular}{|c|c|c|c|c|}
\hline Ligament & Origin & Insertion & Angle & Length/Width \\
\hline Anterior Talofibular & $\begin{array}{l}\text { Anterior aspect of } \\
\text { the distal fibula }\end{array}$ & $\begin{array}{l}\text { Body of the talus, } \\
\text { just anterior to the } \\
\text { articular facet }\end{array}$ & $75^{\circ}$ to the floor & $20 \mathrm{~mm} / 10 \mathrm{~mm}$ \\
\hline $\begin{array}{l}\text { Posterior } \\
\text { Talofibular }\end{array}$ & $\begin{array}{l}\text { Medial surface of } \\
\text { the lateral malleolus }\end{array}$ & $\begin{array}{l}\text { Medially and } \\
\text { horizontally to the } \\
\text { posterior talus }\end{array}$ & & $3 \mathrm{~cm}$ \\
\hline Calcaneofibular & $\begin{array}{l}\text { Anterior border of } \\
\text { the distal fibula just } \\
\text { below the origin of } \\
\text { the ATFL }\end{array}$ & $\begin{array}{l}\text { Small tubercle } \\
\text { posterior and } \\
\text { superior to the } \\
\text { peroneal tubercle of } \\
\text { the calcaneus }\end{array}$ & $104^{\circ}$ with ATFL & $2 \mathrm{~cm} / 5 \mathrm{~cm}$ \\
\hline Deltoid & Medial malleolus & $\begin{array}{l}\text { Talus, calcaneus, } \\
\text { and navicular bones }\end{array}$ & & \\
\hline
\end{tabular}

The muscles of the talocrural joint are important to the function of the ankle joint complex. The eccentric and concentric actions of the muscles are of great importance in injury prevention. The muscles that aid in talocrural joint function are the tibialis anterior, extensor digitorum longus, extensor hallicus longus, peroneus tertius, gastrocnemius and soleus. ${ }^{91}$ In Table B2 a detailed look at the location, action and nerve innervation of the muscles in relation to the talocrural joint is provided.

The muscles of the anterior compartment are ankle dorsiflexors and toe extensors. The muscles are active in walking as they concentrically contract to raise the forefoot to clear the ground during the swing phase of the gait cycle and eccentrically contract to lower the forefoot to the ground after heel strike of the stance phase. ${ }^{91}$ The posterior compartment muscles are plantarflexors. The gastrocnemius and soleus provide the power of plantarflexion that propels the body when walking and plays a major role in running and jumping via push off. ${ }^{91}$ 
Table B2. The Talocrural Joint Muscles. ${ }^{91}$

\begin{tabular}{|c|c|c|c|c|}
\hline Muscle & Origin & Insertion & Innervation & Main Action \\
\hline Tibialis Anterior & $\begin{array}{l}\text { Lateral condyle } \\
\text { and superior } 1 / 2 \text { of } \\
\text { lateral surface of } \\
\text { tibia and } \\
\text { interosseous } \\
\text { membrane }\end{array}$ & $\begin{array}{l}\text { Medial and } \\
\text { inferior surfaces of } \\
\text { medial cuneiform } \\
\text { and base of } 1 \mathrm{st} \\
\text { metatarsal }\end{array}$ & $\begin{array}{l}\text { Deep fibular nerve } \\
\text { (L4, L5) }\end{array}$ & $\begin{array}{l}\text { Dorsiflexes ankle } \\
\text { and inverts foot }\end{array}$ \\
\hline $\begin{array}{l}\text { Extensor } \\
\text { Digitorum Longus }\end{array}$ & $\begin{array}{l}\text { Lateral condyle of } \\
\text { tibia and superior } \\
3 / 4 \text { of medial } \\
\text { surface of fibula } \\
\text { and interosseous } \\
\text { membrane }\end{array}$ & $\begin{array}{l}\text { Middle and distal } \\
\text { phalanges of } \\
\text { lateral four digits }\end{array}$ & $\begin{array}{l}\text { Deep fibular nerve } \\
(\mathrm{L} 5, \mathrm{~S} 1)\end{array}$ & $\begin{array}{l}\text { Extends lateral } \\
\text { four digits and } \\
\text { dorsiflexes ankle }\end{array}$ \\
\hline $\begin{array}{l}\text { Extensor Hallicus } \\
\text { Longus }\end{array}$ & $\begin{array}{l}\text { Middle part of } \\
\text { anterior surface of } \\
\text { fibula and } \\
\text { interosseous } \\
\text { membrane }\end{array}$ & $\begin{array}{l}\text { Dorsal aspect of } \\
\text { base of distal } \\
\text { phalanx of great } \\
\text { toe (Hallux) }\end{array}$ & $\begin{array}{l}\text { Deep fibular nerve } \\
(\mathrm{L} 5, \mathrm{~S} 1)\end{array}$ & $\begin{array}{l}\text { Extends great toe } \\
\text { and dorsiflexes } \\
\text { ankle }\end{array}$ \\
\hline Peroneus Tertius & $\begin{array}{l}\text { Inferior } 1 / 3 \text { of } \\
\text { anterior surface of } \\
\text { fibula and } \\
\text { interosseous } \\
\text { membrane }\end{array}$ & $\begin{array}{l}\text { Dorsum of base of } \\
\text { 5th metatarsal }\end{array}$ & $\begin{array}{l}\text { Deep fibular nerve } \\
(\mathrm{L} 5, \mathrm{~S} 1)\end{array}$ & $\begin{array}{l}\text { Dorsiflexes ankle } \\
\text { and aids in } \\
\text { inversion of foot }\end{array}$ \\
\hline Gastrocnemius & $\begin{array}{l}\text { Lateral head: } \\
\text { lateral aspect of } \\
\text { lateral condyle of } \\
\text { femur. Medial } \\
\text { head: popliteal } \\
\text { surface of femur, } \\
\text { superior to medial } \\
\text { condyle }\end{array}$ & $\begin{array}{l}\text { Posterior surface } \\
\text { of calcaneus via } \\
\text { calcaneal tendon }\end{array}$ & $\begin{array}{l}\text { Tibial nerve (S1, } \\
\text { S2) }\end{array}$ & $\begin{array}{l}\text { Plantarflexes ankle } \\
\text { when knee is } \\
\text { extended; raises } \\
\text { heel during } \\
\text { walking; flexes leg } \\
\text { at knee joint }\end{array}$ \\
\hline Soleus & $\begin{array}{l}\text { Posterior aspect of } \\
\text { head of fibula; } \\
\text { superior quarter of } \\
\text { posterior surface } \\
\text { of fibula soleal } \\
\text { line, medial } \\
\text { border, and of tibia }\end{array}$ & $\begin{array}{l}\text { Posterior surface } \\
\text { of calcaneus via } \\
\text { calcaneal tendon }\end{array}$ & $\begin{array}{l}\text { Tibial nerve (S1, } \\
\text { S2) }\end{array}$ & $\begin{array}{l}\text { Plantarflexes ankle } \\
\text { independent of } \\
\text { position of knee }\end{array}$ \\
\hline
\end{tabular}

The talocrural joint is innervated by the deep fibular nerve in the anterior compartment and accompanied by the anterior tibial artery. ${ }^{91}$ The posterior compartment is innervated by the tibial nerve and accompanied by the posterior tibial artery and the fibular artery and accompanying veins. ${ }^{91}$ 
Subtalar joint: The subtalar joint is formed by articulations between the talus and calcaneus and also converts torque between the leg and foot allowing movements of pronation and supination. ${ }^{1,5,108}$ The subtalar joint is unique in the sense of having two separate joint cavities. The posterior subtalar joint is formed between the inferior posterior facet of the talus and the superior posterior facet of the calcaneus. ${ }^{1,5,108}$ The anterior subtalar joint is formed from the head of the talus, the anterior-superior-facets, the sustentaculum tali of the calcaneus, and the concave proximal surface of the tarsal navicular. ${ }^{1,5,108}$ The anterior and posterior subtalar joints have separate ligamentous joint capsules and are separated from each other by the sinus tarsi and canalis tarsi. ${ }^{1,5,108}$ The ligamentous support of the subtalar joint is extensive and not well understood. A more indepth look at the ligaments is illustrated in Table B3 including origin and insertion.

The ligaments can be divided into deep, peripheral and retinacula. The deep ligaments consist of the cervical and interosseous ligaments. Together theses ligaments stabilize the subtalar joint and form a barrier between the anterior and posterior joint capsules. ${ }^{1,5,108}$ These ligaments, which cross obliquely through the canalis tarsi, have been described as the "cruciate ligaments" of the subtalar joint. The cervical ligament lies within the sinus tarsi and provides support to both the anterior and posterior joints. ${ }^{1,5,108}$ It is the strongest of the subtalar ligaments and has been shown to resist supination. The interosseous ligament because of its diagonal orientation and oblique fiber arrangement across the joint, portions are taut throughout pronation and supination. ${ }^{1,5,108}$ Within the peripheral ligaments the CFL is crucial in preventing excessive inversion and internal rotation of the calcaneus in relation to the talus. ${ }^{1,5,108}$ The fibers of the inferior extensor retinacula (IER) have been proposed to provide support to the lateral aspect of the 
subtalar joint. ${ }^{1,5,108}$ Within the sinus tarsi, three roots of the IER exist including lateral, intermediate, and medial. The lateral root of the IER is the only root that reveals any significant affect on the subtalar joint. ${ }^{1,5,108}$

Table B3. Subtalar Ligaments of the Ankle. ${ }^{1,5,108}$

\begin{tabular}{lll}
\hline Ligament & Origin & Insertion \\
\hline Deep & $\begin{array}{l}\text { Cervical tubercle of the calcaneus } \\
\text { anteriorly }\end{array}$ & Medially to the talar neck \\
Interosseous & $\begin{array}{l}\text { Calcaneus just anterior to the } \\
\text { posterior subtalar joint capsule }\end{array}$ & $\begin{array}{l}\text { Superiorly and medially to the } \\
\text { talar neck }\end{array}$ \\
Peripheral & Anterior border of the distal \\
Calcaneofibular & ATFL & $\begin{array}{l}\text { Small tubercle posterior and } \\
\text { superior to the peroneal tubercle } \\
\text { of the calcaneus }\end{array}$ \\
Lateral Talocalcaneal & Trochlea of the talus & $\begin{array}{l}\text { Lateral surface of the calcaneus } \\
\text { Retinacula }\end{array}$ \\
Inferior Extensor Retinacula & Medial malleolus & Lateral malleolus \\
\hline
\end{tabular}

The muscles of the ankle complex play major roles in concentric and eccentric functions. The peroneus longus and brevis muscles cross the ankle complex which help in the protection against lateral ankle sprains. ${ }^{1,5,108}$ In addition to the peroneals, the muscles of the deep posterior compartment of the leg may also contribute to the dynamic stability of the lateral ankle complex by contracting eccentrically during forced supination of the rearfoot. ${ }^{1,5,108}$ The peroneus longus, peroneus brevis, tibialis posterior, flexor hallicus longus, and flexor digitorum longus muscles are in Table B4 which includes origin, insertion, main action, and nerve innervation. The subtalar joint is innervated by the superficial fibular nerve and tibial nerve..$^{91,108}$ The anterior tibial artery and fibular artery are perforating branches since no one artery courses through the lateral compartment of the subtalar joint. The arteries are accompanied by veins. ${ }^{91,108}$ The posterior tibial artery 
and nutrient artery of the tibia supply the deep posterior compartment along with their accompanying veins. ${ }^{91,108}$

The ankle complex of the subtalar joint is compressed in the lateral and deep posterior compartments. The lateral compartment contains the primary evertors of the foot. ${ }^{91,108}$ Eversion is used to support or depress the medial foot during toe off of the stance phase and to resist inadvertent inversion, preventing injury. ${ }^{91,108}$ The muscles of the deep posterior compartment augment the action through flexion of the digits and support of the longitudinal arch of the foot, along with plantarflexion of the ankle. ${ }^{91,108}$ Table B4. Ankle Complex Muscles of the Subtalar Joint. ${ }^{91,108}$

\begin{tabular}{|c|c|c|c|c|}
\hline Muscle & Origin & Insertion & Innervation & Main Action \\
\hline Peroneus Longus & $\begin{array}{l}\text { Head and superior } \\
2 / 3 \text { of lateral } \\
\text { surface of fibula }\end{array}$ & $\begin{array}{l}\text { Base of } 1^{\text {st }} \\
\text { metatarsal and } \\
\text { medial cuneiform }\end{array}$ & $\begin{array}{l}\text { Superficial fibular } \\
\text { nerve (L5, S1, S2) }\end{array}$ & $\begin{array}{l}\text { Everts foot and } \\
\text { weakly } \\
\text { plantarflexes ankle }\end{array}$ \\
\hline Peroneus Brevis & $\begin{array}{l}\text { Inferior } 2 / 3 \text { of } \\
\text { lateral surface of } \\
\text { fibula }\end{array}$ & $\begin{array}{l}\text { Dorsal surface of } \\
\text { tuberosity on lateral } \\
\text { side of base of } 5^{\text {th }} \\
\text { metatarsal }\end{array}$ & $\begin{array}{l}\text { Superficial fibular } \\
\text { nerve (L5, S1, S2) }\end{array}$ & $\begin{array}{l}\text { Everts foot and } \\
\text { weakly } \\
\text { plantarflexes ankle }\end{array}$ \\
\hline Tibialis Posterior & $\begin{array}{l}\text { Interosseous } \\
\text { membrane; } \\
\text { posterior surface of } \\
\text { tibia inferior to } \\
\text { soleal line; posterior } \\
\text { surface of fibula }\end{array}$ & $\begin{array}{l}\text { Tuberosity of } \\
\text { navicular, } \\
\text { cuneiform, and } \\
\text { cuboid; bases of } 2^{\text {nd }}, \\
3^{\text {rd }} \text {, and } 4^{\text {th }} \\
\text { metatarsals }\end{array}$ & $\begin{array}{l}\text { Tibial nerve (L4, } \\
\text { L5) }\end{array}$ & $\begin{array}{l}\text { Plantarflexes ankle; } \\
\text { inverts foot }\end{array}$ \\
\hline $\begin{array}{l}\text { Flexor Hallicus } \\
\text { Longus }\end{array}$ & $\begin{array}{l}\text { Inferior } 2 / 3 \text { of } \\
\text { posterior surface of } \\
\text { fibula; inferior part } \\
\text { of interosseous } \\
\text { membrane }\end{array}$ & $\begin{array}{l}\text { Base of distal } \\
\text { phalanx of great toe } \\
\text { (Hallux) }\end{array}$ & $\begin{array}{l}\text { Tibial nerve (S2, } \\
\text { S3) }\end{array}$ & $\begin{array}{l}\text { Flexes great toe at } \\
\text { all joints; weakly } \\
\text { plantarflexes ankle; } \\
\text { supports medial } \\
\text { longitudinal arches } \\
\text { of foot }\end{array}$ \\
\hline $\begin{array}{l}\text { Flexor Digitorum } \\
\text { Longus }\end{array}$ & $\begin{array}{l}\text { Medial part of } \\
\text { posterior surface of } \\
\text { tibia inferior to } \\
\text { soleal line; by a } \\
\text { broad tendon to } \\
\text { fibula }\end{array}$ & $\begin{array}{l}\text { Bases of distal } \\
\text { phalanges of lateral } \\
\text { four digits }\end{array}$ & $\begin{array}{l}\text { Tibial nerve (S2, } \\
\text { S3) }\end{array}$ & $\begin{array}{l}\text { Flexes lateral four } \\
\text { digits; plantarflexes } \\
\text { ankle; supports } \\
\text { longitudinal arches } \\
\text { of foot }\end{array}$ \\
\hline
\end{tabular}


Biomechanics

Lateral ankle sprains are one of the most common athletic injuries. Even more concerning is the high recurrence rate after an initial sprain. ${ }^{17}$ Mechanical instability (MI) and functional ankle instability (FAI) have been purposed to be two of the causes of chronic ankle instability (CAI) ${ }^{17} \mathrm{~A}$ change in a joint's axis of rotation is due to abnormal ankle mechanics such as hypermobility and hypomobility resulting in MI. ${ }^{17}$ Proprioceptive, neuromuscular control, strength, and impaired postural control deficits are all causes of FAI. ${ }^{17}$ By examining the normal and abnormal biomechanics of the ankle a better understanding of the relationship between the ankle joint complex and injury can be made.

Kinematics: Motion at one of the joints will cause motion at an adjacent joint, just as a malalignments at one joint may cause compensation at a joint proximal or distal. ${ }^{109}$ Movement of the ankle cannot occur around only one axis. Ankle motion is a combination of dorsiflexion or plantarflexion along with slight internal or external rotation and anterior or posterior translation of the talus on the tibia. ${ }^{110}$

Ankle motion can be described in regard to the planes in which the motion occurs and also the axes around which that movement takes place. During ankle dorsiflexion, the talus glides posteriorly and externally rotates in relation to the mortise. ${ }^{1,17}$ With plantarflexion, the talus glides anteriorly and internally rotates. ${ }^{1,17}$ These motions of the talus in relation to the mortise produce a superior-posterior glide and lateral displacement of the distal fibula in relation to the tibia. ${ }^{1,17}$ The axis of rotation of the talocrural joint passes through the medial and lateral malleoli. In neutral, the axis is slightly anterior to the frontal plane passing through the tibia but slightly posterior to the frontal plane 
passing through the fibula. ${ }^{1,17,92}$ Isolated movement occurs primarily in the sagittal plane, with a small amount of transverse and frontal plane motion occuring about the oblique axis of rotation (anteroposterior axis). ${ }^{1,17,92}$ Considering the position of the malleoli, the axis is laterally rotated 20 to 30 degrees in the transverse plane and slanted about 10 degrees down on the lateral side. ${ }^{92}$ Subtalar joint motion is a complex twisting that is the result of triplanar motion of the talus around a single oblique axis. The subtalar joint has one degree of freedom for pronation and supination. ${ }^{1,17,92}$ Pronation primarily incorporates the cardinal plane motions of eversion and external rotation, while supination primarily involves inversion and internal rotation. ${ }^{1,17,92}$ The subtalar joint has two separate joint cavities that share a common axis of rotation, which averages 42 degrees upward tilt and 23 degrees medial angulation from the perpendicular axes of the foot. ${ }^{1,17,92}$ Ankle range of motion will vary between individuals, but generally, 10 to 20 degrees of dorsiflexion and 20 to 50 degrees of plantarflexion from neutral is considered normal. ${ }^{92,110}$ Inversion and eversion values usually range between 25 to 35 and 5 to 10 degrees, respectively. ${ }^{1,17,110}$ During the stance phase of gait on even ground, the heel strikes with minimal inversion at the subtalar joint followed by eversion ranging from 5 to 10 degrees at $10 \%$ of the walking cycle. ${ }^{1,17,110}$ From there, inversion occurs at the subtalar joint reaching a maximum of 5 degrees at $62 \%$ of the walking cycle. ${ }^{1,17,110}$ Foot position may be abnormal in individuals with altered or deficient proprioceptive ability rendering the foot as an unstable base of support for proximal problems at the knees, hips, pelvis, or low back. The proper biomechanical alignment of the lower extremity, when viewed from an anterior angle, shows a knee that is directly over the base of support and is neither in varus nor valgus. From the lateral view, the 
knee should not be more anterior than the toes during a squat. Also, the leg and back should be parallel during a squat and when landing from a height. ${ }^{111}$

Kinetics: Ankle sprains commonly occur during walking and running but also during lateral cutting and side-shuffle movements and when landing from a jump. ${ }^{21,48}$ Individuals who have functional ankle instability have been shown to have different kinetic and kinematic patterns during jump landings compared with those who do not have FAI. Those with functional ankle instability used more knee flexion range of motion during landing than non-FAI individuals and reach peak ground reaction forces more quickly after landing than non-FAI individuals. ${ }^{31}$ Individuals with functional ankle instability demonstrated significantly less peroneal EMG activity and were in a more inverted position before initial contact. ${ }^{31}$ On landing, the functional ankle instability individuals reached their peak vertical ground reaction force faster than those who did not have FAI ${ }^{31}$ Additionally, individuals with functional ankle instability who exhibited greater angles of ankle inversion during a drop landing were found to use greater cocontraction of the ankle dorsiflexor and plantar flexor muscles, which in turn was associated with greater impact force at initial contact compared with those with nonfunctional ankle instability. ${ }^{31}$ During landing, the body's weight is decelerated using trunk, hip, and leg musculature. ${ }^{12}$ Joints of the lower extremity all work together and are linked by the kinematic chain. A dysfunction at one level will have an effect at a proximal or distal level through the kinetic chain. ${ }^{113}$ During weight acceptance, the subtalar joint moves into pronation in order to absorb shock. While pronated, the foot become more flexible for adjusting to uneven ground surfaces ${ }^{78}$ Pronation is better suited to help the foot maintain balance. When the foot is preparing for push off, a supinated 
and much more rigid foot structure is evident. ${ }^{109}$ In the supinated position, dynamic postural control is more difficult to maintain because of the smaller base of support provided. In individuals with functional ankle instability, the foot tends to supinate more easily resulting in the risk of sustaining a lateral ankle sprain. ${ }^{78,109}$

Ankle position during acceleration and maximum velocity sprinting is important in two realms, joint stability and force production. ${ }^{78,109,112} \mathrm{~A}$ dorsiflexed position of the ankle is superior in both aspects. ${ }^{78,109,112}$ Anatomically, the dorsiflexed ankle is more stable due to the skeletal construction of the joint, contraction of the plantar flexors, and the pre-stretched gastrocnemius and soleus. ${ }^{46,78,109,112,114}$ This stability allows the ankle joint to be resistant to collapse upon ground contact, making short ground contact times at high velocities achievable. ${ }^{46,78,109,112,114}$ Elite sprinters and jumpers seldom vary greatly from 90 degrees during the approach. ${ }^{46,78,109,112,114}$

The pre-stretch on the gastrocnemius and soleus offer opportunity for necessarily quick elastic force generation through tendon reflexes upon ground contact. Without introducing greater degrees of plantar flexion upon push-off a delay in toe-off can hamper recovery efforts. ${ }^{46,78,109,112,114}$ Also, since the gastrocnemius originates above the knee, keeping the muscle on stretch by dorsiflexing the ankle allows assistance in any voluntary knee flexion during recovery, thus allowing the hamstrings to remain relaxed. $^{46,78,109,112,114}$

Stability of the ankle is of the utmost importance in acceleration, sprinting, and jumping. A stable ankle provides a solid base through which the hip and knee joints may apply force to the ground. During acceleration, touchdown occurs on the ball of the foot, and excessive collapse in the ankle joint means force will be absorbed rather than 
transmitted to the ground. ${ }^{46,78,109,112,114}$ As velocities increase, the ankle is stabilized in a slightly plantarflexed position, to facilitate this force application by the ball of the foot through the stable ankle. ${ }^{46,78,109,112,114}$ In either case, the ankle remains stable and the ball of the foot acts as a fulcrum and point of force application as the tibia pivots forward, rather than allowing the ankle to collapse and the tibia to pivot forward at the calcaneus. ${ }^{46,78,109,112,114}$

During the final stages of support, a quick force contribution occurs by way of the tendon reflex. The fact that many quadruped mammals use the ankle in a similar fashion, can give us insight into the correct function of the ankle joint during locomotion. ${ }^{46,78,109,112,114}$

Conversely, a plantar flexed ankle position results in joint instability and creates longer contact times, delaying toe-off and disrupting the location of the base of support with respect to the center of mass..$^{46,78,109,112,114}$ Because of this instability, some collapse of the ankle joint upon contact is inevitable, and a compensatory plantar flexion occurs as a substitute force producer. ${ }^{46,78,109,112,114}$ Another compensating strategy involves the ankle joint stabilizing upon contact by firing early, out of sequence. ${ }^{46,78,109,112,114}$

It is important that any contribution by the ankle be quick, elastic, and reflexive in nature. ${ }^{46,78,109,112,114}$ Excessive contribution by plantar flexion at toe-off results in delayed recovery, because the plantar flexors are primarily composed of slow twitch muscle fiber. ${ }^{46,78,109,112,114}$ An unstable ankle joint also delays hip and knee extension because establishment of a firm base of support occurs too late, resulting in poor push-offs and takeoffs. ${ }^{46,78,109,112,114}$ Because of time restrictions and reflexes that occur at high speeds, 
it is not possible to recruit ankle stabilizers at selected points. ${ }^{46,78,109,112,114}$ Proper ankle positioning must be constantly maintained.

Compressive forces will vary throughout phases of gait as well as position of the foot and ankle. ${ }^{109}$ In normal bilateral weight bearing, 77 to 90 percent of the force from the body's weight is transmitted through the tibial plafond to the talar dome. ${ }^{92}$ The remaining 10 to 23 percent is placed on the medial or lateral facets. ${ }^{92}$ When inversion is increased, the force upon the medial facet is increased as is the force on the lateral facet during eversion. ${ }^{92,93}$ In the early stance phase of gait, the compressive forces transmitted to the ankle are less than 20 percent of body weight. ${ }^{92,93}$ The compressive forces can be as high as five times body weight during the late stance phase of normal gait. ${ }^{92,93} \mathrm{In}$ addition, a shear force of eight times body weight can act upon the ankle during heel off. $^{92,93}$

Structural foot type may affect postural sway during static and dynamic postural control. ${ }^{78}$ Hyperpronated and hypersupinated subtalar joints may be less able to control postural sway. ${ }^{78}$ Orthotics have shown to significantly reduce postural sway in those with a lateral ankle sprain. ${ }^{78}$ An increased structural support to both the medial and lateral sides of the foot occurs with wearing orthotics. This support does not allow the increased subtalar joint movement that occurs in lateral ankle sprains. ${ }^{115}$ Epidemiology

The most common sports-related injury is an ankle sprain, more specifically lateral ankle sprains. An estimated 23,00 to $27,000^{1,2,3,4}$ ankle sprains occur per day in the United States, which equals approximately one ankle sprain per $10,000^{1,2,3,4}$ people daily. Seven percent to 10 percent of the ankle sprains account for emergency room visits. ${ }^{4,5}$ 
However, an estimated 55 percent of individuals suffering an ankle sprain never seek medical treatment. ${ }^{1}$

Studies have shown that approximately 10 percent to 25 percent $^{4,5,6,7,88}$ of all sports injuries are ankle sprains. Ankle sprains account for 75 percent of all ankle injuries with 85 percent due to inversion trauma ${ }^{6,9,10,11}$ Due to the inversion mechanism of injury predominantly 80 percent to 95 percent affect the lateral ligaments of the ankle., ${ }^{9,12,13,14}$ After suffering one ankle sprain, an individual is predisposed to chronic ankle instability. The reoccurrence rate of ankle sprains varies in the literature from 20 percent to as high as 80 percent. $^{3,6,7,13,15,16,17,18,19,20}$

Ankle sprains result in more time loss than any other single injury in athletics. Time loss due to ankle injuries is estimated to account for one sixth of all time lost from sport. Safran et al. ${ }^{7}$ stated that approximately 25 percent of time loss in football, basketball, and cross country is due to ankle injuries.

The prevalence of ankle sprains in sport is incredibly significant, especially with competition and practice combined. Player contact in games accounts for 58.0 percent of injuries while 41.6 percent of player contact in practices results in injury. ${ }^{115}$ The lower extremity is accountable for 53.8 percent of the injuries in games and 53.7 percent in practices. ${ }^{115}$ Since 1998, the NCAA used the Injury Surveillance System to understand the epidemiology of injuries via sport and gender. ${ }^{116}$ Eight men's teams in the NCAA participated in the study including baseball, basketball, football, ice hockey, lacrosse, soccer, wrestling, and spring football. ${ }^{116}$ For ankle ligament sprains, men's baseball accounted for 7.9 percent of all injuries, basketball 26.6 percent, football 13.6 percent, ice hockey 4.5 percent, lacrosse 14.4 percent, soccer 17.2 percent, wrestling 7.4 percent, and 
finally spring football 14.9 percent. ${ }^{116}$ Overall the frequency of ankle sprains/strains was 19,256 , with a distribution of 13.1875 percent at a rate of 0.7425 per 1000 athleteexposures. ${ }^{116}$ Eight women's teams also participated in the study including basketball, field hockey, gymnastics, ice hockey, lacrosse, soccer, softball, and volleyball. ${ }^{116}$ For ankle ligament sprains, women's basketball accounted for 24 percent of all injuries, field hockey 10 percent, gymnastics 15.4 percent, ice hockey, 2.8 percent, lacrosse 17.7 percent, soccer 16.7 percent, softball 9.9 percent, and volleyball 23.8 percent. ${ }^{116}$ Women had a fairly decreased overall frequency of 7,861 compared to the 19,256 of the men, a distribution rate of 15.0375 percent at a rate of $0.76625 .{ }^{116}$

Fong et al. ${ }^{117}$ reported on ankle injuries for 43 sports in many different countries. Ankle sprains were the most common injury (33 sports, $76.7 \%$ ), followed by fracture ( 7 sports, $16.3 \%$ ). ${ }^{117}$ Of the 43, the fourteen most common American sports (American football, basketball, cheerleading, field hockey, gymnastics, ice hockey, lacrosse, rugby, indoor soccer, outdoor soccer, softball, tennis, track and field and volleyball) had a weighted percentage of ankle sprains ranging from 25.1 to 100 percent. ${ }^{117}$ In some sports, all of the reported ankle injuries were ankle sprains (100\%). These sports included Australian rules football, field hockey, handball, orienteering, scooter and squash. ${ }^{117}$

In addition to these six sports, ankle sprain percent- age was also $>80 \%$ in indoor volleyball, American football, martial arts, basketball, aeroball, ultimate frisbee, flag football, cheerleading, indoor soccer, ice hockey, lacrosse, badminton and netball. ${ }^{117}$ Etiology

Lateral ankle sprains and recurrent lateral ankle sprains are the most frequent injury substained by athletes. Recurrent lateral ankle sprains have been hypothesized as 
causes of mechanical instability (MI) and functional instability (FAI was reported earlier). Mechanical instability refers to laxity of a joint due to structural damage to ligamentous tissues, which support the joint, and functional instability stems from neuromuscular and proprioceptive deficits. ${ }^{1,10,19,29,30,31}$

When a lateral ankle sprain occurs, structural damage not only occurs to the ligamentous tissue, but also to the nervous and musculotendinous tissue around the ankle complex. ${ }^{1,10,19,29,30,31}$ The lateral ankle ligaments and joint capsule of the talocrural and subtalar joints have been shown to be extensively innervated by mechanoreceptors. A disruption of the sensory receptors within the lateral ligamentous structures is believed to result in a decreased ability to sense changes in joint position. . $^{1,10,19,29,30,31,32}$ Mechanoreceptors are most active in the sensation of joint movements near the ends of ranges of motion. Mechanoreceptors sense increased tension in the ligaments and send an afferent message to the spinal cord. In response, an efferent response is sent to the muscles; which can slow or reverse the direction of joint movement. ${ }^{1,10,19,29,30,31,32}$

While injury to the ligaments may result in laxity of the joints of the ankle complex, neuromuscular deficits are also likely to occur due to the injury to the nervous and musculotendinous tissue..$^{1,10,19,29,30,31}$ These neuromuscular deficits may be manifested as impaired balance, reduced joint position sense, slower firing of the peroneal muscles to inversion perturbation of the ankle, slowed nerve conduction velocity, impaired cutaneous sensation, strength deficits and decreased dorsiflexion range of motion. ${ }^{1,10,19,29,30,31}$

Many acute lateral ankle sprains occur when performing a lateral cutting movement, or landing on an irregular surface, such as the foot of another player, however 
recurrent lateral ankle sprains are liable to suffer the same mechanism due to the neuromuscular deficits of the ankle complex..$^{10,16,18,19,29,30,31}$ When landing on an irregular surface or poorly executing a cut, a large supination torque that supinates and inverts the rearfoot excessively results in overloading and damaging the lateral ankle ligaments. ${ }^{16}$ The mechanism of injury is in direct relationship to foot positioning and initial contact of the rearfoot during gait or landing. ${ }^{1,7,11,16,88,94}$ An inconsistency has been found in the literature as to whether the ankle is in dorsiflexion or plantarflexion when the foot makes initial contact with the ground. Hertel ${ }^{1}$ however, suggested that increased plantarflexion at initial contact appears to increase the likelihood of suffering a lateral ankle sprain.

Excessive inversion and internal rotation of the rearfoot, coupled with external rotation of the tibia, results in strain to the lateral ligaments. ${ }^{1,7,11,16,88,94}$ If the strain in any of the ligaments exceeds the tensile strength of the tissues, ligamentous damage occurs. ${ }^{1,7,11,16,88,94}$ However, when the ankle internally rotates in relationship to the tibia, predictability of the progression of tears in sequence results. ${ }^{1,7,11,16,88,94}$ The anterior talofibular ligament, the anterolateral capsule, and the distal tibiofibular ligament are the first ligaments to tear in a lateral ankle sprain. ${ }^{1,7,11,16,88,94}$ Continuous strain on the lateral ankle will cause the calcaneofibular ligament to tear. ${ }^{1,7,11,16,88,94}$ With extreme force on the lateral ankle, the posterior talofibular ligament ruptures, which is occasionally associated with an ankle dislocation, distal lateral malleolar avulsion or spiral fracture, medial malleolar fracture, or talar neck or medial compression fractures. ${ }^{1,7,11,16,88,94}$ Lateral ankle sprains occur in sports with a high level of jumping, running, and cutting and in which involve optimal neuromuscular development and control, balance, and quick stop-start movements. ${ }^{6,10,88,118}$ With the high-intensity nature of sports many factors 
may predispose an athlete or individual to ankle sprains, more specifically lateral ankle sprains. Intrinsic characteristics that may lead to ankle sprains are as follows; demographics, ${ }^{11}$ gender,${ }^{119,120}$ height and weight, ${ }^{119}$ limb dominance,,${ }^{119}$ previous sprain, ${ }^{119}$ ligamentous stability, ${ }^{11,119}$ range of motion, ${ }^{119}$ muscular strength, ${ }^{11,119}$ foot size,${ }^{119}$ anatomic foot and ankle alignment, ${ }^{11,119}$ postural sway, ${ }^{11,119}$ gait mechanics, ${ }^{11}$ and muscle reaction time. ${ }^{11,119}$ Some extrinsic risk factors include ankle bracing and taping, ${ }^{119}$ shoe type,${ }^{119}$ duration and intensity of competition, ${ }^{119,120}$ sport, ${ }^{120}$ and player position. ${ }^{119}$

Two added characteristics can explain the frequency of occurrences involving the lateral ankle. Looking at the anatomy of the ankle, the lateral malleolus projects more distally than the medial malleolus producing less bony obstruction to inversion than eversion. ${ }^{1,711,16,89,94}$ Also the deltoid ligament is a much stronger ligament than any of the lateral ankle ligaments combined. . $^{1,711,16,86,94}$

Assessment Tools

The most common mechanism of injury is an athlete who "rolled" over the outside of the ankle..$^{4,68,95,121,122,123}$ The injury can occur from contact or from noncontact such as landing on a plantarflexed-inverted foot. The main site of pain and swelling is usually localized to the lateral side of the ankle over the ATFL. ${ }^{4,68,95,121,122,123}$ Most patients have difficulty walking due to pain and discomfort. Ecchymosis can occur 24 to 48 hours after injury. ${ }^{4}$ The discoloration is usually along the lateral side but can also occur in the retrocalcaneal bursal area and along the heel because of the potential space available for swelling and the pooling effect of gravity. ${ }^{4,68,95,121,122,123}$

Many studies suggest performing clinical stability tests for ligamentous laxity/damage between four and seven days after the injury. ${ }^{44,68,95,121,122,123}$ The theory is 
that the acute pain and swelling will be diminished and the patient will be able to relax during the examination. Delaying the grading of the severity of an ankle sprain might prevent diagnosis. The anterior drawer test (AD), talar-tilt inversion test (TT) and the medial subtalar glide test are all stability tests for the lateral ankle complex.

The ATFL is the most commonly injured ligament of the ankle. The anterior drawer test evaluates the integrity of the ATFL by the amount of anterior-talar displacement that can be produced in the sagittal plane. ${ }^{44,68,95,121,123}$ To perform the test, the patient is seated with the knee flexed to 90 degrees and the involved foot relaxed in slight plantarflexion. ${ }^{44,68,95,121,123}$ The examiner stabilizes the tibia and fibula with one hand and grasps the calcaneus with the other. ${ }^{44,68,95,121,123}$ This may also be performed with the patient in a prone position. ${ }^{44,68,95,121,123}$ While the examiner assures stabilization of the distal tibia and fibula, an anterior force is applied to the calcaneus and talus. 44,68,95,121,123 A positive sign for a possible ATFL sprain is anterior translation of the talus away from the ankle mortise that is greater on the involved side, as opposed to the noninvolved side. ${ }^{44,68,95,121,123}$ The sensitivity of the test can be improved by placing the ankle in 10 degrees of plantarflexion. $44,68,95,121,123$

Vela et al. ${ }^{95}$ stated that the anterior drawer test been has shown to have a sensitivity ranging from 32 percent to 80 percent without discrimination of the lateral ligaments affected by injury. However, for isolated tears of the ATFL, the anterior drawer test has been reported to have a sensitivity of 60 percent and a specificity of 74 percent. Stiell et al. ${ }^{124}$ and Fujil et al, ${ }^{125}$ on the other hand, have reported poor intertester reliability of the anterior drawer test, ${ }^{95}$ due to the subjectivity and experience of the examiner. 
The talar tilt inversion test evaluates the integrity of the CFL by the angle produced by the tibial plafond and the dome of the talus in response to forceful inversion of the hindfoot. ${ }^{4,68,95,121,122}$ The talar tilt inversion test is performed by having the patient lie on the uninvolved side on a table with the involved foot relaxed and the knee flexed to 90 degrees. ${ }^{4,68,95,121,122}$ The examiner stabilizes the distal tibia with one hand while grasping the talus with the other. ${ }^{4,68,95,121,122}$ The examiner first places the foot in the anatomical position (neutral). The examiner then tilts the talus into an adducted position. 4,68,95,121,122 A positive test reveals range of motion in the adducted position on the involved foot greater than that of the noninvolved foot. ${ }^{4,68,95,121,122}$ This may be indicative of a tear of the CFL of the ankle. ${ }^{4,68,95,121,122} \mathrm{~A}$ general rule is more than ten degrees of difference from the noninvolved side is considered abnormal. ${ }^{4,68,95,121,122}$ Vela et al. ${ }^{95}$ stated that the talar tilt inversion test produced the greatest CFL strain when the test was performed in ten degrees of dorsiflexion. Due to performing the test in a more plantarflexed position greater strain of the ATFL would occur. The clincial evidence for the CFL evaluation is inadequate to make definitive recommendations concerning physical examination of isolated CFL lesions. The anterior drawer test appears to be a reasonably good test with a reported sensitivity of 68 percent when evaluating combined lesions of the ATFL and CFL. ${ }^{95}$

Very little research investigating the role of physical examination techniques in the diagnosis of subtalar injuries exist in the literature. The medial subtalar-glide test is reported to test the laxity of the subtalar joint. ${ }^{4,95}$ The test is performed with the patient in the same position as the anterior drawer, seated and knee flexed to 90 degrees. The examiner stabilizes the tibia and fibula with one hand and grasps the plantar surface of 
the foot with the other hand. ${ }^{4,95}$ The examiner translates the calcaneus medially (inversion) on the talus in the transverse plane. ${ }^{4,95}$ A positive sign is increased laxity on the involved compared to the uninvolved. ${ }^{4,95}$ The sensitivity and specificity of this test is unknown. ${ }^{4,95}$ However, Vela et al. ${ }^{95}$ stated that Hertel et al. ${ }^{15}$ has found good agreement between the results of the medial subtalar-glide test and those of subtalar stress images in patients. Denegar et al. ${ }^{127}$ also reported significantly greater laxity with the medial subtalar-glide test in the injured limb compared to the uninjured limb.

Two objective measures of ankle laxity and range of motion is the ankle arthrometer and the goniometer. An ankle arthrometer provides instrumented measurement of the load-displacement characteristics of the ankle complex. ${ }^{128,129}$ The ankle arthrometer is a method for assessing translatory and uniplanar rotary displacement of the foot in relation to the leg that result from the combined motions within the talocrural and subtalar joints. ${ }^{128,129}$ Kovaleski et al. ${ }^{129}$ found a high reliability coefficient between intratester and intertester trials ( $\mathrm{ICC}=.80$ to .97$)$ and a low standard of error measurement demonstrating small measurement differences between testers.

Visual estimation is commonly used in clinical practice and is very insufficient and unreliable. ${ }^{63,64,65}$ Menadue et al. ${ }^{63}$ stated that inter-observer reliability for visual estimation of active plantarflexion and dorsiflexion is very low. Menadue et al.$^{63}$ used the goniometric measurement protocol for inversion and eversion. Menadue and colleagues found within session intra-observer reliability high to very high ranging from ICC $=0.82$ to 0.96 . No significant difference was found in ICC scores between position or directions of movement. Between-session intra-observer reliability ranged from low to high (ICC $=0.42$ to 0.80 ) but highest for inversion. Goniometric measurements of inversion 
were moderately well correlated between the prone and sitting positions. Overall, reliability of standardized measurement of ankle inversion and eversion range of motion was variable within observers, between observers and between-sessions.

Rome and Coweison ${ }^{64}$ examined intratester and intertester reliability for goniometric measurements of ankle dorsiflexion (ADF) and ankle plantar flexion (APF) active range of motion (AROM). For intratester reliability of measurements obtained with the universal goniometer (UG), intraclass correlation coefficients (ICC) for all physical therapists were 0.64 to 0.92 (median, 0.825) for ADF and 0.47 to 0.96 (median, 0.865) for APF. Intertester reliability was quantified with use of ICCs. Measurements obtained by UG were 0.28 for ADF and 0.25 for APF; ICC of visual estimation (VE) for ADF was 0.34 and was 0.48 for APF. For parallel-forms intratester reliability obtained with UG and VE ranged from 0 to 0.94 (median, 0.58) for ADF and 0 to 0.86 (median, 0.625) for APF. Thus, a physical therapist may use a goniometer when making repeated measurements of ankle joint AROM. Considerable inconsistency exists when two or more physical therapists make repeated goniometric and visual measurements of ankle motion on the same subject.

Traditional Rehabilitation

In chronic ligament injury, the normal healing process of connective tissue is disrupted. Normal tissue remodeling is prevented by stresses and hypovascularity. ${ }^{24,25,26,27}$ Connective tissue tends to become shorter and denser as it heals, unless it has beneficial conditions. ${ }^{24,25,26,27}$ This contracture and thickening partially results from the increased interfiber bonding in a shortened state. ${ }^{24,25,26,27}$ The individual 
fibers lose gliding capacity and mobility relative to each other, as do bundles of fibers, and whole ligaments relative to their surrounding structures. ${ }^{24,25,26,27}$

The adhesion process of fibers and layers of tissue leads to reduced extensibility and mobility. ${ }^{24,25,26,27}$ With poor circulation, hypoxia, and continued tearing, tissue regrowth takes the form of scar tissue as tears coalesce in portions of the ligament. ${ }^{24}$ Scar tissue is weaker than the normal fiber. ${ }^{24,25,26,27}$ The water content and amount of ground substance of the tissue is reduced. There is a lessening of a normal ratio of ground substance to collagen fibers, which is another factor causing a denser, less compliant structure and impaired metabolism of the tissue. ${ }^{24,25,26,27}$ By-products of tissue degeneration and breakdown can form deposits in the ligament, disrupting function. ${ }^{24,25,26,27}$

In chronic injury the normal bi-directional fiber arrangement is disorganized, which reduces extensibility and strength. Prolonged low-grade inflammation is maintained along with disturbed lymphatic and vascular flow. ${ }^{24,25,26,27}$ This also causes abnormal metabolic function in the tissue. ${ }^{24,25,26,27}$ Ligaments can exhibit both hypo- and hyper-mobility. ${ }^{24,25,26,27}$ Often, both of these conditions exist in different portions of the same structure. ${ }^{24,25,26,27}$ There is a disruption of balanced neural activity, as the ligament receptors report inappropriate strain signals caused by the irritation of the tissue. ${ }^{24,25,26,27}$ This causes an ongoing stress on the ligament due to muscular tension, uncoordinated movement, and joint instability. All these factors contribute to general neurotrophic disturbance and impairment of circulation in the tissue..$^{24,25,26,27}$

In the literature, variations of chronic ankle instability ${ }^{34,35}$ rehabilitation have been reported. Guidelines can be established for a rehabilitation program; but it is important 
that a cookbook approach be avoided. ${ }^{25}$ Rather the development of a rehabilitation plan is highly individualized based on the particular needs and demands of the sport. This is largely due to the many factors that enter into each athlete's situation to be able to apply a standard treatment regimen for all. Once a complete understanding of the injury, including knowledge of mechanism, major anatomical structures affected, severity, and injury healing phases, then guidelines for a rehabilitation program can be established.

Hertel and Denegar ${ }^{130}$ described a hierarchy of rehabilitation goals including the neuromuscular control paradigm. The hierarchy of rehabilitation goals is a pyramid incorporating the phases of healing from phase one to return to full participation. (Figure B1)

Figure B1. Hierarchy of rehabilitation goals. ${ }^{130}$

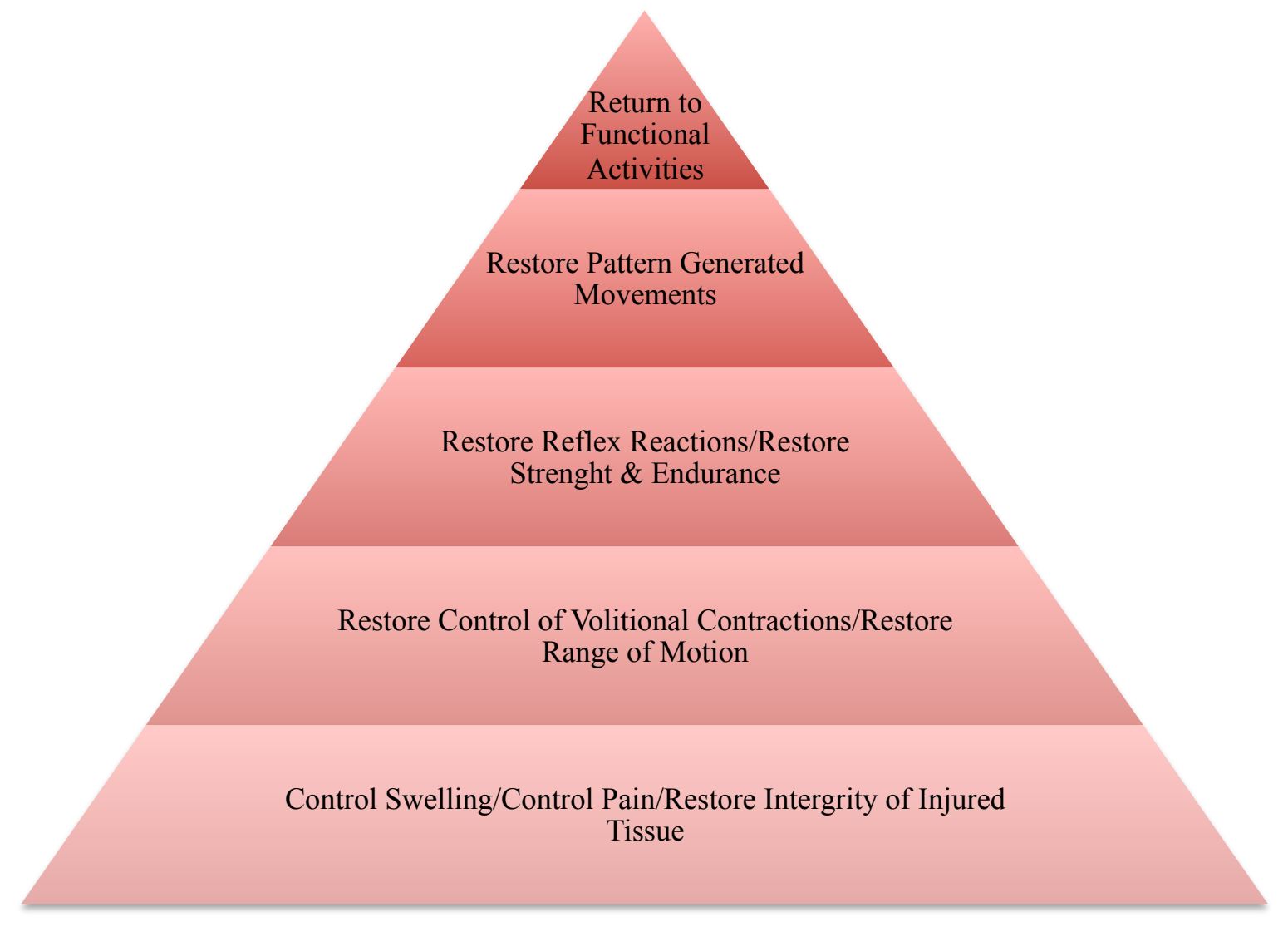


The foot of the pyramid begins phase one or the acute inflammatory response phase which begins immediately and can last up to four days. ${ }^{725,96,97}$ In the initial inflammatory phase, erythrocytes and inflammatory cells, particularly neutrophils, enter the site of injury. ${ }^{24,25}$ In the first 24 hours, monocytes and macrophages predominate, and phagocytosis of necrotic materials occurs. Vasoactive and chemotactic factors are released with increased vascular permeability, initiation of angiogenesis, stimulation of fibrocytes proliferation, and recruitment of more inflammatory cells. ${ }^{24,25}$ The fibrocytes gradually migrate to the injury site, and type III collagen synthesis is initiated. ${ }^{24,25}$ In essence, the inflammatory stage of healing controls and cleans up of the injured tissues, thus creating an environment that is conducive to the fibroblastic repair. ${ }^{7,24,25,96,97}$ The primary focus of rehabilitation during this phase is to control swelling and to modulate pain by protection of the injured tissues. Cryotherapy, ${ }^{131,132,133}$ compression, ${ }^{90}$ and elevation ${ }^{134}$ along with medication and modalities such as transcutaneous electrical nerve stimulation are used in various combination to achieve these goals. ${ }^{7,27,96,97,126,128,129,131,132,133,134}$ Strategies to control pain and swelling must be minimized and managed before a rehabilitation program can progress beyond the restoration of volitional muscle control.

Once pain and swelling are maintained and controlled, efforts to restore range of motion and restoration of control of volitional muscle contractions my be initiated, provided that the healing tissues are not compromised by the exercises. ${ }^{126}$ This is phase two or the fibroblastic repair phase which begins once the inflammatory response has subsided. ${ }^{7,24,25,96,97}$ During this stage of the healing process, synthesis of type III collagen is peaking due to the fibroblastic cells laying down a matrix of collagen fibers. ${ }^{24,25}$ 
However, as the type III collagen fibers are receding, the type I collagen synthesis process is commencing. ${ }^{7,24,25,27,96,97}$ The water content and glycosaminoglycan concentrations remain high for proliferation of fibroblasts, collagen synthesis and fibrogenesis, formulation of granulation tissue, and dense fibrous scar formation. ${ }^{24,25,27}$ Phase two may begin as early as four days after the injury and may last for several weeks. ${ }^{7,24,25,27,96,97}$ Modality use during this phase is used exclusively to maintain and control pain and swelling. ${ }^{7,96,97}$ Therefore, treatments such as cryotherapy ${ }^{131,132,133}$ and electrical stimulation are acceptable. ${ }^{96,97}$

The next goal of rehabilitation should be the restoration of volitional muscle contractions, restoration of range of motion, ${ }^{128,129}$ and maintain cardiovascular fitness. ${ }^{128,129}$ Not all athletic injuries result in significant loss of one's ability to control muscle contractions. ${ }^{130}$ However, volitional control of muscle contraction should be assessed as needed prior to progressing the athlete into strength and functional retraining. ${ }^{130}$ At the second lower tier of the hierarchy of rehabilitation goals pyramid towel stretches, ${ }^{128,129,136}$ slant board, ${ }^{128,129,136}$ alphabet drawing, ${ }^{128,129}$ wobble board, ${ }^{31,48,83,128,129,136,137}$ and BAPS $^{31,48,83,128,129,136,137}$ board are all exercises that can be incorporated to increase control of volitional muscle contractions and range of motion.

The third tier from the top of the hierarchy of rehabilitation goals pyramid focuses on the restoration of reflex reactions and restoration of strength and endurance. This is the third phase or maturation-remodeling and is the longest of the three and may last for three weeks up to one year, depending on the severity of the injury. ${ }^{7,24,25,96,97}$ After approximately six weeks, the maturation-remodeling phase commences with the healing tissue only about 30 percent healthy. ${ }^{24,25,27}$ During this stage, the healing tissue is resized 
and reshaped. A corresponding decrease in cellularity, collagen and glycosaminoglycan synthesis occurs. ${ }^{24,25}$ The maturation-remodeling phase can be divided into a consolidation and maturation stage. The consolidation stage commences at about six weeks and continues up to ten weeks. In this period, the repair tissue changes from cellular to fibrous. Fibrocyte metabolism remains high during this period, and fibrocytes and collagen fibers become aligned in the direction of stress. ${ }^{24,25} \mathrm{~A}$ high proportion of type I collagen is synthesized during this stage. After ten weeks, the maturation stage occurs, with gradual changes of fibrous tissue to scar-like tissue over the course of one year. ${ }^{24,25}$ During the latter half of this stage, fibrocyte metabolism and vascularity decline. $^{24,25}$

The priorities in the rehabilitation program include improving reflex responses and strengthening. Closed kinetic chain activities that maximize input from joint mechanoreceptors are ideal for retraining reflex responses and sensorimotor facilitation in the lower extremity. ${ }^{127}$ Single- and double-leg; wobble board, ${ }^{31,48,83,128,129,136,137}$ BAPS board, ${ }^{31,48,83,128,129,136,137}$ Dyna Disk, ${ }^{31,48,83,128,129,136,137}$ Airex, ${ }^{31,48,83,128,129,136,137}$ mini squats, ${ }^{31,48,83,128,129,136,137}$ Star Excursion Balance Test, ${ }^{20,71,130}$ and leg press $^{31,48,83,128,129,136,137}$ are but a few examples of closed kinetic chain activities that can help enhance reflex responses. Having eyes opened and closed, uncompromised surfaces, object toss, and adding rhythmic stabilization, can increase the difficulty of the exercise. ${ }^{31,48,83,128,129,136,137}$ However, open kinetic chain activities facilitate in maximizing joint position sense.

Hale et al. ${ }^{34}$ in a 4 -week rehabilitation program found subjects with chronic ankle instability demonstrated deficits in postural control and SEBT reach tasks of the involved 
limb compared to the uninvolved limb and reported functional deficits of the involved limb compared to healthy subjects. Following rehabilitation, the chronic ankle instabilityrehabilitation group had greater SEBT reach improvements on the involved limb than the other groups and greater improvements in FADI and FADI-Sport scores. Hale et al. ${ }^{34}$ concluded that postural control and functional limitations exist in individuals with chronic ankle instability. In addition, rehabilitation appears to improve these functional limitations. Finally, there is evidence to suggest the SEBT may be a good functional measure to monitor change after rehabilitation for chronic ankle instability.

McKeon et al. ${ }^{35}$ found that a 4 -week progressive balancing-training program that emphasized dynamic stabilization after a landing from a hop, in a variety of directions and conditions significantly improved self-reported functional status, static postural control assessed by measures of time-to-boundary magnitude and variability, and dynamic postural control as assessed with SEBT reach distance.

Strength training can be accomplished in various ways, and programs should be tailored to the athlete's own needs. ${ }^{130}$ In general, high-repetition/low resistance exercises should precede high-resistance exercises and power work. ${ }^{130}$ Restoration of muscular endurance is vital for returning to unrestricted sports performance. ${ }^{130}$ Muscular strength, endurance, and power are among the most essential factors in restoring the function of a body part to pre-injury status. ${ }^{29,48,83,96,97,128,129,137}$ Isometric, isotonic (progressive resistive), isokinetic, and plyometric exercises can be beneficial in rehabilitation..$^{29,96,97,130}$ A major goal in performing the exercises is to work through a full range of motion. ${ }^{31,48,83,128,129,136,137}$ A functional rehabilitation-strengthening plan should involve exercises in all three plane of motion, concentrate on a combination of concentric, 
eccentric, and isometric exercises designed to increase strength through a full multiplanar range of motion. ${ }^{31,48,83,128,129,136,137}$ Strengthening exercises are very intricate in their specific functions. For instance isometric 4-way ankle, ${ }^{29,31,48,83,128,129,130,136,137}$ isotonic 4way ankle (adding tubing, bands, cuff weights), ${ }^{29,31,48,83,128,129,130,136,137}$ toe raises, ${ }^{29,31,48,83,128,129,130,136,137}$ toe walks, ${ }^{29,31,48,83,128,129,130,136,137}$ toe curls, ${ }^{29,31,48,83,128,129,130,136,137}$ heel walks, ${ }^{29,31,48,83,128,129,130,136,137}$ marble pickup, ${ }^{29,31,48,83,128,129,130,136,137}$ PNF, ${ }^{29,31,48,83,128,129,130,136,137}$ and box jumping (different heights $)^{29,31,48,83,128,129,130,136,137}$ all function to provide resistance to a particular muscle, group of muscles, or ligament structure.

As the injured athlete progresses through the lower three tiers of the hierarchy of rehabilitation goals pyramid, the athlete can progress to the restoration/retraining of pattern-generated movements. Pattern-generated movements involve a series of gradual progressive activities designed to prepare the individual for return to a specific sport. $^{7,96,97,130}$ Pattern-generated movements can be as complex or simple as needed. The athlete may perform at different levels for each of these functional sequences. ${ }^{7,96,97,130}$

The restoration of pattern-generated movements is copious and also highly individualized. Examples of which are a running progression, walking, ${ }^{128,129,130}$ walk/jog, ${ }^{128,129,130}$ jogging, ${ }^{128,129,130}$ jog/run, ${ }^{128,129,130}$ and running, ${ }^{128,129,130}$ double-tosingle leg hop, ${ }^{128,129,130}$ figure 8's running and hopping, ${ }^{128,129,130}$ stair running (one-to-two steps), ${ }^{128,129,130}$ and skipping. ${ }^{128,129,130}$ Activities should be specific to the athlete's sport such as kicking a ball, jumping for a pass, running routes, or rebounding. All exercise are performed at various speeds and intensities. $7,128,129,130$ 
Finally the upper tier, return to functional activity is reached. ${ }^{7,96,97}$ However, before considering returning an athlete to full participation, the athlete should have full, pain free range of motion, at least 80 to 90 percent of pre-injury strength, and if full practice is tolerable without insult to the injured part, the athlete may return to competition. ${ }^{7,96,97,138}$ A general guideline for functional exercise is that the athlete should be able to do tomorrow what was completed today. If not, this means the rehabilitation is being forced too rapidly given the state of the healing tissues.

Unlike training, retraining calls for great respect of pain. Pain during activities that alters movement patterns indicates that the healing tissues are being stressed. In other words, it is too soon to attempt this particular exercise at this stage of recovery. In addition, excessive stress to healing tissues can lead to an increase in pain later. ${ }^{130}$ Graston Instrumented-Assisted Soft Tissue Mobilization

In the early 1990's the Graston Instrumented-Assisted Soft Tissue Mobilization (GISTM) technique was researched at Ball State Memorial Hospital and Ball State University in Muncie, Indiana. ${ }^{99}$ In 1994, the technique became the cornerstone for treatment of chronic and acute injuries at the TherapyCare Resources out-patient clinic in Indianapolis. Data was gathered in a five year period following adaptation of the GISTM. $^{99}$

The developer of the technique created the instruments out of necessity. After suffering a severe knee injury while water skiing and failing to respond to surgery and conventional therapies, the technique was developed. With the assistance of an elite track and football athlete, and combined knowledge of the musculoskeletal system with a professional background as a tool and die marker, the instruments were designed. ${ }^{99}$ 
Graston Instrumented-Assisted Soft Tissue Mobilization (GISTM) is an advanced form of soft tissue mobilization combined with targeted stretching and strengthening exercises that is used to detect and release scar tissue, adhesions, and fascial restrictions. ${ }^{36,37,38,39,40,41,50,51,52}$ The technique uses patented stainless steel instruments that are designed to adapt to the various contours of the body. ${ }^{36,37,38,39,40,41,50,51,52}$ GISTM is an advancement of soft tissue mobilization (STM) and cross friction massage that allows the clinician to detect and treat soft tissue dysfunctions in a precise and specific manner. $36,37,38,39,40,41,50,51,52$

The patented stainless steel instruments are specially designed with beveled edges $^{36,37,38,39,40,41,50,51,52}$ utilizing concavity and convexity by increasing the mechanical advantage of a lever to enhance the clinician's ability to perform GISTM. The instruments are utilized in a multidirectional stroking fashion applied to the skin at 30 to 60 degree angles at the treatment site..$^{38,51,52}$ This application allows the clinician to detect irregularities in the soft tissue texture through "tactile feedback." ${ }^{, 52}$ With the gliding of the instruments a controlled amount of microtrauma to the area occur producing inflammation and the initiation of the healing response. $36,37,38,39,40,41,50,51,52$

The controlled microtrauma causes microvascular trauma and capillary hemorrhage that induces a localized inflammatory response. ${ }^{36,37,40,41,50}$ It is believed that the beveled edges and angulation of strokes is thought to trigger the localized inflammatory response as a result of contacting areas of fibrosis. ${ }^{98}$ The inflammatory response is the first step in initiating the body's healing cascade. ${ }^{36,37,40,41,50}$ The tissue then moves from an inactive degenerative state to an active inflammatory stage; when blood platelets and fibrin begin filling the damaged tissue and fibroblasts and phagocytic 
cells migrate to the injury site. ${ }^{36,37,40,41,50}$ A progression into the proliferative stage occurs as fibroblasts increase in number and synthesize collagen. Fibroblasts initiate production of fibronectin. ${ }^{36,37,40,41,50}$ A reduction in cellularity and realignment of collagen fibers begin the remodeling stage and the shift of immature Type III collagen to mature Type I collagen as fibronectin production decreases. ${ }^{36,37,40,41,50}$

A unique characteristic of tendon healing is proliferation of fibroblasts. Davidson et al. ${ }^{36}$ and Gehlsen et al. ${ }^{37}$ both found a difference in fibroblast proliferation in induced achilles tendon injuries in rats using Augmented Soft Tissue Mobilization (ASTM) technique as an adjunct to traditional rehabilitation. The hallmark sign of a tendon injury is collagen fiber disruption and misalignment, which were present in both the Davidson et al. ${ }^{36}$ and Gehlsen et al. ${ }^{37}$ studies. However, there was no evidence of phase three or the maturation-remodeling stage of healing taking place in either of the studies by Davidson et al. ${ }^{36}$ and Gehlsen et al. ${ }^{37}$ Davidsion et al. ${ }^{36}$ stated that the explanation for realignment during the maturation-remodeling stage is unclear. ${ }^{36,37}$ In recent studies, the issue of mechanical force eliciting cellular responses has gained support. ${ }^{6,37}$ Gehlsen et al. ${ }^{37}$ stated that mechanical stimuli have been shown to alter many cellular functions including ion transport, release of second messengers, protein synthesis, secretion, and even gene expression. The mechanical stimulation applied to cells by GISTM may play an imperative role in modifying cellular functions thus leading to fibroblast proliferation and increased activation. $^{37}$

In spite of this, the Davidson et al. ${ }^{36}$ and Gehlsen et al. ${ }^{37}$ studies are the only two experimental studies that elicit objective findings. Several case reports have been conducted using soft tissue mobilization techniques. The case reports were performed on 
humans not animals thus the plethora of subjective and inadequacy of objective data. In addition, majority of the soft tissue mobilization studies focus on tendon injuries.

Five clinical trial studies, over the past eight years, have been conducted using the ASTM or GISTM technique. Sevier and colleagues ${ }^{40}$ conducted a trial of ASTM on a patient suffering for two years from chronic lateral epicondylitis. The patient's pain and disability significantly decreased with an increase in strength. The patient has since discontinued the use of the tennis elbow brace as well. Wilson and colleagues ${ }^{49}$ performed ASTM on twenty patients with patellar tendinitis. Sixty percent of the traditional rehabilitation group symptoms and pain resolved and 100 percent of the ASTM group had complete resolution of symptoms and pain. The remaining 40 percent from the traditional rehabilitation group, crossed-over to the ASTM group and 50 percent of the crossover group had resolution of symptoms and pain. ${ }^{40}$ Hammer and Pfefer ${ }^{50}$ treated a patient diagnosed with subacute lumbar compartment syndrome using GISTM. ${ }^{50}$ The patient after several treatments was asymptomatic, had nonrestrictive active and passive range of motions in all directions, normal functional tests and finally had a significant improvement in hamstring and right external rotators flexibility. Aspegren and colleagues $^{51}$ used GISTM on a patient suffering from costochondritis. There was a decrease in pain and a significant improvement in the Dallas Pain Questionnaire from the initial visit. Lastly, Burke and colleagues ${ }^{52}$ conducted a pilot study on twenty-two patients with carpal tunnel syndrome using both the GISTM and ASTM techniques. There was an increase in nerve conduction latencies, increased wrist strength, increased range of motion and had greater patient satisfactory ratings. ${ }^{52}$ 
There have been no experimental studies conducted on GISTM technique and its effects on chronic ankle sprains. Most of the clinical studies and case reports that have been conducted have used animals and some human subjects for lateral epicondylitis, ${ }^{40}$ patellar tendinopathy, ${ }^{49}$ rotator cuff tendinopathy ${ }^{36}$ and achilles tendinopathy. ${ }^{36,37,38,39,40,41}$ Melham ${ }^{41}$ completed a case report on chronic ankle pain and fibrosis using the augmented soft tissue mobilization technique (ASTM) and found pain ceased with activity, the subjects range of motion improved, surgical scar matured, and the excessive fibrotic connective tissue around the ankle softened and diminished. Loghmani and colleagues ${ }^{45}$ conducted a study on rats using the GISTM technique in an attempt to examine the biomechanical and histological effects of instrumented-assisted cross fiber massage (IACFM) on early and long-term ligament healing. Twenty-one rats received nine treatments over a four-week period and thirty received thirty treatments over twelve weeks. At four and twelve weeks the ligaments were pulled to tensile failure. At four weeks a 31 percent increase in strength was found with 34 percent stiffer ligament structure compared to the untreated ligament. Following twelve weeks a 15.4 percent stiffer ligament was found. A histological exam of the ligaments treated with IACFM were found to have greater fibroblast proliferation and more regular collagen alignment at both four or twelve weeks. In spite of this, no study has furthered evaluated the effects of chronic ankle sprains and the condition of the ligaments after such an injury. Ultrasonography

Ultrasonography is an imaging method that uses high-frequency sound waves to produce precise images of structures within the body. ${ }^{100}$ This imaging technique is a valuable complementary clinical tool enabling clinicians to improve the accuracy of their 
diagnostic skills and management decisions essential for maintaining the highest standards of patient care. ${ }^{83}$

Ultrasonography has a number of advantages over other imaging methods, including non-invasiveness, safety, rapidity of performance, inexpensive, ability to scan multiple joints, repeatability, ability to do dynamic evaluations, and high patient acceptability. ${ }^{67,100,101,102,103,104}$ Despite the many advantages, ultrasonography is operator dependent ${ }^{67,100,101,102,103,104}$ and has an inherent learning curve. ${ }^{67}$

Studies have found that ultrasonography is effective in diagnosing ankle ligament injuries, most notably those affecting the anterior talofibular ligament (ATFL). Van Dijk et al. ${ }^{68}$ reported ultrasonography with a 92 percent sensitivity and 64 percent specificity for diagnosing injuries to the lateral ankle ligaments. Unfamiliarity of the technique, anatomy and pathology of assessing the lateral ankle complex can lead to a major limitation and inconvenience. Therefore, knowledge of the correct positioning of the ultrasonography probe is imperative. The ATFL inserts from the anterior aspect of the talus, therefore, the probe is placed in a slightly oblique position from the malleolus toward the forefoot. ${ }^{67,100,101,102,103,104}$ The ligament is hyperechoic when the fibers are perpendicular to the ultrasound beam ${ }^{67,100,101,102,103,104}$ Being approximately two $\mathrm{mm}$ thick, during examination, the ATF ligament must be straight and tight from one insertion point to the other. ${ }^{67,100,101,102,103,104}$ The PTFL runs from the posterior part of the malleolus to the posterior part of the talus, and is difficult to see on ultrasonography, being partially or sometimes completely hidden by the malleolus. ${ }^{67,100,101,102,103,104}$ The CFL forms the middle portion of the lateral collateral ligament and is between the inferior part of the lateral malleolus and the calcaneus, and runs in a slightly posterior oblique 
direction toward the calcaneus. ${ }^{67,100,101,102,103,104}$ The CFL is approximately two-three mm thick and is hyperechoic in the distal two-thirds only because of the obliquity of the proximal part. ${ }^{67,100,101,102,103,104}$ When examining, the ankle must be dorsiflexed to allow the ligament to be seen more clearly. ${ }^{67,100,101,102,103,104}$ With sufficient knowledge of ankle technique, anatomy, and pathology, ultrasonography will be a reliable and effective means for detecting injury, especially that of ligamentous structures.

Visual Analogue Scale (VAS) of Pain

Accurate estimation of the patient's pain is crucial in the process of pain relief. However, pain assessment is a difficult and complex task. Unlike other physiological conditions for which severity can be assessed with quantitative measures, there is no medical test or physiological index that provides an objective measure of pain. ${ }^{58,59}$

Traditionally, the strategy to measure pain has been to use intensity scales anchored by number and/or words descriptive of different degrees of pain. ${ }^{58,59,60,61,62}$ However, these scales have often been criticized for their lack of sensitivity. ${ }^{58,59,60,61,62}$

Over the decades, the visual analogue scale (VAS) has come to be one of the most popular methods for measurement of pain intensity. ${ }^{58,59,60}$ The VAS consists of a ten $\mathrm{cm}$ line anchored by two extremes of pain, usually "no pain" and "unbearable pain. $" 58,59,60,61,62$ The patient is asked to make a vertical mark through the line corresponding to the intensity of pain. ${ }^{58,59,60,61,62}$ The scale is scored by measuring the distance in mm from "no pain" to the patient's mark. ${ }^{58,59,60,61,62}$ Simple and reproducible, the VAS has been shown to yield valid and reliable pain estimates; furthermore, several studies have demonstrated that VAS is usually more sensitive than traditional descriptive pain scales for detecting fine changes in pain levels. ${ }^{58,59,60,61,62}$ 
Of great significance, at least for research purposes, is the fact that the VAS provides ratio data and permits the use of parametric statistics. ${ }^{58,59,60,61,62}$ Test-retest reliability has been reported to be high and concurrent validity has been found to be moderate for the VAS. ${ }^{58,59,60,61,62}$ The VAS is considered a strong, clinically useful, reliable and valid measure of pain intensity. ${ }^{58,59,60,61,62}$

However, several disadvantages of the VAS have been pointed out. First, the VAS is physically awkward for patients who have perceptual/motor problems that preclude marking the line with a pen. ${ }^{58,59,60,61,62}$ Second, the VAS does not provide an immediate result that can be readily documented. ${ }^{58,59,60,61,62}$ Third, a significant correlation between the number of incorrect responses to the VAS and age appears to exist. ${ }^{58,59,60,61,62}$ Fourth, measurement errors as simple as changes in the length of the line from photocopying can occur. ${ }^{58,59,60,61,62}$ Finally, several studies have shown that some patients have difficulty in understanding the VAS; however, providing adequately clear instructions can minimize this problem. ${ }^{58,59,60,61,62}$ Foot and Ankle Ability Measures Questionnaire

An assessment of an injury should include both objective and subjective measurements. Patient-assessed measures would provide a feasible and appropriate method for addressing concerns of the patient ${ }^{105}$ during rehabilitation. Researchers believe that both psychological and psychosocial factors are related to the development of chronic health problems and determine the level of disabilities and participation problems. ${ }^{105}$ Therefore, in chronic health problems like chronic ankle instability, the degree of present impairments, disabilities, participation problems and decreased quality of life should be documented from the patient's perspective. ${ }^{105}$ 
The Foot and Ankle Ability Measures of Activities of Daily Living (FAAM ADL) and FAAM Sport are designed to meet the need for a self-reported evaluative instrument that comprehensively assesses physical function of individuals with musculoskeletal disorders of the leg, foot, and ankle. ${ }^{56}$ The FAAM is a region-specific self-report of function with two components just like the Functional Ankle Disability Index (FADI) ${ }^{55,56}$ The FAAM ADL is 21 -item questionnaire related to activities of daily living and the FAAM Sport incorporates eight more items that evaluate perceived disability due to foot or ankle injury in activities associated with physical activity and sport participation. ${ }^{55,56}$

Martin et al. ${ }^{56}$ reported that the FAAM ADL and FAAM Sport are reliable, valid, and responsive measure of self-reported physical function. Validity evidence was provided for test content, internal structure, score stability, and responsiveness. Test retest reliability was 0.89 and 0.87 for the ADL and Sports subscales, respectively. ${ }^{56}$ The minimal detectable change based on a 95 percent confidence interval was \pm 5.7 and \pm12.3 points for the ADL and Sports subscales, respectively. ${ }^{56}$ Two-way repeated measures ANOVA and ROC analysis found both the ADL and Sport subscales were responsive to changes in status $(p<0.05) .{ }^{56}$ The minimal clinically important differences were 8 and 9 points for the ADL and Sport subscales. ${ }^{56}$ Guyatt responsive index and ROC analysis found the ADL subscale was more responsive than general measures of physical function while the Sports subscale was not. ${ }^{56}$ Then Carcia et $\mathrm{al}^{57}$ reported that for both the ADL and Sports subscales, FAAM scores were greater in healthy participants $(100 \pm 0.0$ and $99 \pm 3.5)$ than in subjects with CAI $(88 \pm 7.7$ and $76 \pm 12.7 ; \mathrm{P}<.001) .{ }^{57}$ Similarly, for both ADL and Sports subscales, FAAM scores were greater in athletes who 
indicated that their ankles were normal $(98 \pm 6.3$ and $96 \pm 6.9)$ than in those who classified their ankles as either nearly normal, or abnormal $(87 \pm 6.6$ and $71 \pm 11.1 ; \mathrm{P}<.001) .{ }^{57}$ Carcia et $\mathrm{al}^{57}$ found relationships between FAAM scores and self-reported global ratings of function for both ADL and Sports subscales. Relationships were stronger when all athletes, rather than just those with CAI, were included in the analyses. Compared with other instruments, the FAAM ADL and FAAM Sport are measures specific to the leg, foot and ankle that show promise in quantifying function among a young, active population with chronic ankle instability. ${ }^{56,57}$

The directions consisted of instructions to answer every question with one response that most closely described their condition within the past week. ${ }^{55,56}$ If the activity in question is limited by something other than the foot or ankle, N/A is marked. Activity is rated as no difficulty at all (4 points), slight difficulty (3 points), moderate difficulty (2 points), extreme difficulty (1 point), unable to do (0 points), or N/A (not applicable). For pain related to the foot and ankle, patients select no pain (4 points), mild (3 points), moderate ( 2 points), severe ( 1 point), or unbearable ( 0 points) ${ }^{55,56}$ The FAAM scores are recorded as a percentage of 84 points. ${ }^{556}$ The FAAM Sport scores are recorded as a percentage of 32 points. ${ }^{55} 56$ Additionally, the patient is asked to rate their current level of function during usual ADLs and sport activities from 0-100 percent. ${ }^{56}$ Summary

Ankle sprains are a common injury among any population. The intricate anatomy of the ankle includes articulations, muscles, tendons, ligaments, and nerves. Mechanics of the talocrural and subtalar joint provide an understanding of mechanical and functional ankle instability. The prevalence of ankle injuries is common in sports and accounts for 
the most time lost than any other single injury alone. Many intrinsic and extrinsic factors may predispose an individual to ankle sprains. A common cause of a lateral ankle sprain occurs from a large supination torque, excessive supination and inversion of the rearfoot, lateral cutting movements, or landing on irregular surfaces are other causes. The anterior drawer test, talar tilt inversion test, medial subtalar glide test, and the ankle arthrometer have been found sufficient in prognosis of ankle injuries, specifically those of the ATFL and CFL. Many variations of ankle injury rehabilitation programs have been reported. This is due to each program being highly individualized but still incorporating the basic principles of range of motion, sensorimotor facilitation, strength, and functional activity. Studies have provided sufficient evidence of increased range of motion, increased neovascularization, collagen alignment, scar tissue remodeling and a decrease in pain and disability. Ultrasonography has been found effective in diagnosis of ankle ligament injuries with high sensitivity and moderate specificity. However, ultrasonography is very operator dependent and has a lengthy learning process. The VAS pain scale is one of the most popular methods and is considered strong, clinically useful, reliable and valid in measure of pain intensity. Finally, the foot and ankle ability measures is unique in that it is population-specific to athletes, objective, and depicts a patient's perspective. 
Table C1. Parental Consent Form

THE EFFECTS OF A RANDOMIZED FOUR-WEEK GRASTON INSTRUMENTEDASSISTED SOFT TISSUE MOBLIZATION DYNAMIC BALANCING-TRAINING PROGRAM ON INDIVIDUALS WITH CHRONIC ANKLE INSTABILITY.

\section{Co-Investigator(s);}

"Jessica L. Schaefer, BS, ATC"

\section{Contact Persons}

In the event your child experiences any side effects or injury related to his research, you should contact Jessica Lynn Schaefer, ATC at (812) 686-0973 or at jschaef3@mix.wvu.edu. If you have any question, concerns, or complaints about this research, you can contact Dr. Michelle A. Sandrey, PhD, ATC at (304) 293- 3295 Ext. 5220 or at msandrey@mail.wvu.edu.

For information regarding my rights as a research subject, I may contact the Executive Secretary of the Review Board at (304) 293-7073.

\section{Introduction}

You have been asked to allow your child to participate in this study, which has been explained to you and your child by Jessica Lynn Schaefer, BS, ATC. This study is being conducted by the Principle Investigator, Michelle A Sandrey, PhD, ATC and the CoInvestigator, Jessica L. Schaefer, BS, ATC in the College of Physical Activity and Sport Sciences at West Virginia University. This research is being conducted to fulfill the requirements for a thesis in Athletic Training in the College of Physical Activity and Sport Sciences at West Virginia University under the supervision of Michelle A. Sandrey, $\mathrm{PhD}, \mathrm{ATC}$.

\section{Purpose of Study}

The purpose of this study aims to investigate the affects of Graston InstrumentedAssisted Soft Tissue Mobilization (GISTM) technique and its influence on improving range of motion, neovascularization, collagen alignment, pain and disability in individuals experiencing chronic ankle instability following a four-week dynamic balancing-training program. 


\section{Description of Procedures}

\section{Procedures}

This study will be conducted at University High School, 991 Price Street, Morgantown, WV 26505 or West Virginia University Athletic Training Research Lab, PO Box 6116, Morgantown, WV 26505. All testing and training procedures will be done in the athletic training room and gymnasium so that subjects may hear and understand all directions in a quiet atmosphere that is free from any distractions.

At an orientation meeting the purpose of this study will be explained to your child. Your child will be given a demographic questionnaire (gender, height, past and current medical history) and have special tests done for inclusion/exclusion into the study. This will take approximately twenty minutes to complete. Your child does not have to answer all the questions. You will have the opportunity to see the questionnaire before singing this parental consent and Authorization to Use or Disclose Protected Health Information forms. All complete forms will be confidential. If your child is an eligible subject, he/she child will be contacted by the primary researcher and will schedule a meeting time for approximately one hour for further testing using the Foot and Ankle Ability Measures (FAAM) and FAAM Sport, Visual Analog Scale (VAS), range of motion (ROM), Ultrasonography (US), and Star Excursion Balance Test (SEBT). Your child will then be instructed on the tests that he/she will be asked to perform.

Your child will have a one in three chance of being randomly assigned to either one of the treatment/balancing-training groups or the control group. Your child will then be asked for his/her full cooperation and to work to the best of their ability two times a week for forty-five minutes over a four-week period. Your child will complete a final sixtyminute post-testing session for the FAAM and FAAM Sport, VAS, ROM, US, and SEBT, which will conclude his/her participation. The post-test will be performed to the exact specifications as the pre-test, and will be completed within the week following the final treatment/balancing-training session of the fourth week.

After your child's level of functional ankle instability is determined by the demographic questionnaire. The special tests will be the final inclusion or exclusion criteria into the study. If your child has a grade III sprain determined by a board certified orthopedic surgeon he/she will be excluded from the study.

Your child will be asked to answer the FAAM-26 item questionnaire with one response that will most clearly describe his/her condition within the past week. Upon completion, your child will answer the FAAM Sport 8-item questionnaire with one response that will most clearly describe his/her condition within the past week.

Your child will then be asked to make a mark on the $10 \mathrm{~cm}$ line that will accurately describe his/her pain level at that instant.

Ankle range of motion will be taken of your child. He/she will be instructed to sit, with their knee off the edge of the table for the first two measurements. The primary 
researcher will instruct your child to move his/her ankle up and down as far as possible. Your child will then be asked to lay face down on the table with their ankle off the end of the table. He/she will be instructed to move their foot in and out as far as possible. Your child will next have ultrasonography performed on his/her ankle over the anterior talofibular ligament (ATF) and the calcaneofibular ligament (CF). Your child will be instructed to lie down on the table with his/her knee straight and ankle pointing down and in to image the ATF ligament. Next, your child will be instructed to move his/her ankle up and in to image the CF ligament. The images of your child's ankle ligaments will be stored and saved under their identification number that will be randomly assigned to keep records confidential.

Your child's true leg length will be measured use a tape measure to normalize data. Instructions will be given to your child to stand in the middle of a grid formed by three lines extending out at $135^{\circ}$ from each other. Your child will be asked to reach as far as possible along the three lines [anterior (A), posteromedial (PM), and posterolateral (PL)] lines, make a light touch on the line, and return the reaching leg back to the center, while maintaining a single-leg stance with the other leg in the center of the grid.

Your child will be allowed to practice reaching in each of the three directions three times. Following a 5-min rest period, your child will perform three trials in each of the three directions. The reach distance will be recorded from the center of the grid to the point of maximum distance by the reach leg.

Interventions

Your child will perform a ten-minute dynamic Flex Band flexibility warm-up routine regardless of the experimental or control group he/she is randomly assigned. The dynamic warm-up will consist of ankle, calf, achilles, hamstring, groin, I-T Band, and concludes with quadriceps/hip flexor stretches on both legs.

If your child is randomly assigned to the Graston Instrumented-Assisted Soft Tissue Mobilization (GISTM) experimental group or GISTM-Sham experimental group, he/she will first receive an eight to ten minute treatment. Your child will be instructed to lay face done with their feet over the edge of the table. An emollient will be rubbed onto his/her leg to prevent friction. The primary researcher will then take the tools and gently move up and down your child's leg. After the bottom of the foot and calf have been worked with the tools, your child will be instructed to lie on the table face up. Again emollient will be rubbed onto your child's leg. The primary researcher will again move up and down your child's leg and foot. Finally, your child will be instructed to lay on his/her side with a pillow between their legs for support, again emollient will be applied to their leg. The primary researcher will now take the tools and work around your child's ankle. After the GISTM treatment the tools will be cleaned with SaniCide, a hard surface disinfectant. Once your child's treatment is completed or if your child is in the control experimental group, the primary researcher will now instruct your child in the dynamic balancingtraining program. 
The dynamic balancing-training program is a four-week progressive program for those with chronic ankle instability. Your child will be shown a video that demonstrates the exercises in their entirety. He/she will perform single-limb hops to stabilization, hop to stabilization and reach, unanticipated hop to stabilization, and single-limb stance activities into a rehabilitation program.

The first exercise will require your child to hop in four directions ten times. Each repetition will consist of a hop from the starting position to the target position at 18, 27, or 36 inches. After your child stabilizes his/her balance in a single-limb stance, he/she will hop in the exact opposite direction back to the starting position and stabilize in the single-limb stance.

The second exercise will be a five-repetition hop to stabilization and reach exercise with a combination of the previously mentioned exercise. Your child will hop, stabilize, and reach back to the starting position, then hop back to the starting position and reach to the targeted position.

The third exercise, will be an unanticipated hop to stabilization with your child standing in the middle of a nine-marker grid. A randomized, sequence of numbers will be displayed on a computer screen in front of your child. Each number will correspond to a hop target position. As the progression of numbers change, your child will hop to the new target position. Your child will do three repetitions.

The fourth and final exercise is the single-limb stance. Your child will perform three repetitions of a single-limb stance. The exercise is made more difficult by the environment and conditions, such as eyes open, eyes closed, standing on foam, and throwing a ball.

The four-week dynamic balancing-training program has several levels of difficulty. Your child will be progressed accordingly. At the end of the fourth week FADI and FADI Sport, VAS, ROM, US, and SEBT measurements will be taken again. Your child will now complete the study and will be released from this study.

\section{Risks and Discomforts}

I understand there are no known or expected risks for my child from participation in this study. I understand that my child will be exposed to a stress x-ray by a board certified orthopedist and ultrasonography by a board certified ultrasonographer in this study. I understand mild muscle soreness in my child's injured lower extremity may occur with performing the GISTM treatment. The treatment will only occur every other day. Dynamic balancing-training program exercises will be the only known or expected discomforts with performing the GISTM and dynamic balancing-training program. I understand that my child will be doing dynamic stretching before treatment for the dynamic balancing-training program to reduce muscle soreness, but should be minimal at worst. After completion of the dynamic balancing-training program ice will be applied to the injured extremity to also aid in prevention of muscle soreness. While performing any 
testing, or exercise I understand that my child will not lose their balance because they will be performing the test with their eyes opened. However, I understand that my child will be instructed to touch down with the non-injured foot during single-limb balancing exercises if they feel they are losing their balance.

I understand that every precaution has been taken to prevent my child from being injured in this study. If an adverse physical or psychological reaction were to occur during any point of the study, appropriate care or referral will be made available. Should any injury occur to my child, I understand that Jessica L. Schaefer, BS, ATC will provide first aid and make any necessary medical referrals at my expense.

\begin{abstract}
Alternative
Your child does not have to participate in this study. No negative actions will be taken against your child if they choose not to participate. At the present time there are no other alternatives to consider.
\end{abstract}

\title{
Benefits
}

Your child may not receive any direct benefit from this study. The knowledge gained from this study may eventually benefit others.

\section{Financial Considerations}

I understand that my child will not receive any monetary compensation for participation in this study.

\section{Confidentiality}

Any information about your child that is obtained as a result of participation in this research will be kept as confidential as legally possible. Your child's research records and test results, just like hospital records, may be subpoenaed by court order or may be inspected by federal regulatory authorities without your additional consent. In addition, there are certain instances where the researcher is legally required to give information to the appropriate authorities. These would include mandatory reporting of infectious diseases, mandatory report of information about behavior that is imminently dangerous to your child such as suicide, child abuse, etc. In any publications that result form this research neither our child's name nor any information form, which your child might be identified, will be published without your consent.

\section{Voluntary Participation}

Participation in this study is voluntary. You or your child may refuse to participate in this study. You or your child may withdraw from this study at any time. Refusal to participate or withdraw will not affect your child's future care, or your child's class standing or grades, as appropriate and will involve no penalty to you or your child 
position on the team. In the event new information becomes available that may affect your willingness to allow your child to participate in this study this information will be given to you so that you can make an informed decision about whether or not to continue your child's participation. You have been given the opportunity to ask questions about the research, and have received answers concerning areas you did not understand.

Upon signing this form I will receive a copy.

I willingly consent to allow my child to participate in this research.

$\overline{\text { Signature of Parent/Guardian }} \overline{\text { Printed Name }} \quad$ Date/Time

The parent/guardian has had the opportunity to have questions addressed. The parent/guardian willingly agrees to allow his/her child to be in the study.

$\overline{\text { Signature of Co-Investigator }} \overline{\text { Printed Name }} \quad$ Date/Time


THE EFFECTS OF A RANDOMIZED FOUR-WEEK GRASTON INSTRUMENTEDASSISTED SOFT TISSUE MOBLIZATION DYNAMIC BALANCING-TRAINING PROGRAM ON INDIVIDUALS WITH CHRONIC ANKLE INSTABILITY.

\section{Co-Investigator(s);}

"Jessica L. Schaefer, BS, ATC"

\section{Contact Persons}

In the event you experience any side effects or injury related to his research, you should contact Jessica Lynn Schaefer, ATC at (812) 686-0973 or at jschaef3@mix.wvu.edu. If you have any question, concerns, or complaints about this research, you can contact Dr. Michelle A. Sandrey, PhD, ATC at (304) 293- 3295 Ext. 5220 or at msandrey@mail.wvu.edu.

For information regarding my rights as a research subject, I may contact the Executive Secretary of the Review Board at (304) 293-7073.

\section{Introduction}

You have been asked to participate in this study, which has been explained to me by Jessica Lynn Schaefer, BS, ATC. This study is being conducted by the Principle Investigator, Michelle A Sandrey, $\mathrm{PhD}$, ATC and the Co-Investigator, Jessica L. Schaefer, BS, ATC in the College of Physical Activity and Sport Sciences at West Virginia University. This research is being conducted to fulfill the requirements for a thesis in Athletic Training in the College of Physical Activity and Sport Sciences at West Virginia University under the supervision of Michelle A. Sandrey, PhD, ATC.

\section{Purpose of Study}

The purpose of this study aims to investigate the affects of Graston InstrumentedAssisted Soft Tissue Mobilization (GISTM) technique and its influence on improving range of motion, neovascularization, collagen alignment, pain and disability in individuals experiencing functional ankle instability following a four-week dynamic balancing-training program.

\section{Description of Procedures}

\section{Procedures}

This study will be conducted at University High School, 991 Price Street, Morgantown, WV 26505 or West Virginia University Athletic Training Research Lab, PO Box 6116, Morgantown, WV 26505. All testing and training procedures will be done in the athletic training room and gymnasium so that subjects may hear and understand all directions in a quiet atmosphere that is free from any distractions. 
At an orientation meeting the purpose of this study will be explained to me. I will be given a demographic questionnaire (gender, height, past and current medical history) and have special tests done for inclusion/exclusion into the study. This will take approximately twenty minutes to complete. I do not have to answer all the questions. I will have the opportunity to see the questionnaire before singing this assent and Authorization to Use or Disclose Protected Health Information forms. All complete forms will be confidential. If I am an eligible subject, I will be contacted by the primary researcher and will schedule a meeting time for approximately one hour for further testing using the Foot and Ankle Ability Measures (FAAM) and FAAM Sport, Visual Analog Scale (VAS), range of motion (ROM), Ultrasonography (US), and Star Excursion Balance Test (SEBT). I will then be instructed on the tests that I will be asked to perform.

I will have a one in three chance of being randomly assigned to either one of the treatment/balancing-training groups or the control group. I will then be asked for my full cooperation and to work to the best of my ability two times a week for forty-five minutes over a four-week period. I will complete a final sixty-minute post-testing session for the FAAMand FAAM Sport, VAS, ROM, US, and SEBT, which will conclude my participation. The post-test will be performed to the exact specifications as the pre-test, and will be completed within the week following the final treatment/balancing-training session of the fourth week.

After my level of functional ankle instability is determined by the demographic questionnaire. The special tests will be the final inclusion or exclusion criteria into the study. If I have a grade III sprain determined by a board certified orthopedic surgeon I will be excluded from the study.

I will be asked to answer the FAAM-26 item questionnaire with one response that will most clearly describe my condition within the past week. Upon completion, I will answer the FAAM Sport 8-item questionnaire with one response that will most clearly describe my condition within the past week.

I will then be asked to make a mark on the $10 \mathrm{~cm}$ line that will accurately describe my pain level at that instant.

My ankle range of motion will be taken. I will be instructed to sit, with my knee off the edge of the table for the first two measurements. The primary researcher will instruct me to move my ankle up and down as far as possible. I will then be asked to lay face down on the table with my ankle off the end of the table. I will be instructed to move my foot in and out as far as possible.

I will next have ultrasonography performed on my ankle over the anterior talofibular ligament (ATF) and the calcaneofibular ligament (CF). I will be instructed to lie down on the table with my knee straight and ankle pointing down and in to image the ATF ligament. Next, I will be instructed to move my ankle up and in to image the CF ligament. The images of my ankle ligaments will be stored and saved under my identification number that will be randomly assigned to keep records confidential. 
My true leg length will be measured to normalize data. Instructions will be given to me to stand in the middle of a grid formed by three lines extending out at $135^{\circ}$ from each other. I will be asked to reach as far as possible along the three lines [anterior (A), posteromedial (PM), and posterolateral (PL)] lines, make a light touch on the line, and return the reaching leg back to the center, while maintaining a single-leg stance with the other leg in the center of the grid.

I will be allowed to practice reaching in each of the three directions three times. Following a 5-min rest period, I will perform three trials in each of the three directions. The reach distance will be recorded from the center of the grid to point of maximum distance by my reach leg.

\section{Interventions}

I will perform a ten-minute dynamic Flex Band flexibility warm-up routine regardless of the experimental or control group I will be randomly assigned. The dynamic warm-up will consist of ankle, calf, achilles, hamstring, groin, I-T Band, and concludes with quadriceps/hip flexor stretches on both legs.

If I am randomly assigned to the Graston Instrumented-Assisted Soft Tissue Mobilization (GISTM) experimental group or GISTM-Sham experimental group, I will first receive an eight to ten minute treatment. I will be instructed to lay face done with my feet over the edge of the table. An emollient will be rubbed onto my leg to prevent friction. The primary researcher will then take the tools and gently move up and down my leg. After the bottom of my foot and calf have been worked with the tools, I will be instructed to lie on the table face up. Again emollient will be rubbed onto my leg. The primary researcher will again move up and down my leg and foot. Finally, I will be instructed to lay on my side with a pillow between my legs for support, again emollient will be applied to my leg. The primary researcher will now take the tools and work around my ankle. After GISTM treatment the tools will be cleaned with SaniCide, a hard surface disinfectant. Once my treatment is completed or if I am in the control experimental group, the primary researcher will instruct me in the dynamic balancing-training program.

The dynamic balancing-training program is a four-week progressive program for those with chronic ankle instability. I will be shown a video that demonstrates the exercises in their entirety. I will perform single-limb hops to stabilization, hop to stabilization and reach, unanticipated hop to stabilization, and single-limb stance.

The first exercise will require me to hop in four directions ten times. Each repetition will consist of a hop from the starting position to the target position at 18, 27, or 36 inches. After I stabilize my balance in a single-limb stance, I will hop in the exact opposite direction back to the starting position and stabilize in the single-limb stance.

The second exercise is a five-repetition hop to stabilization and reach exercise and will be a combination of the previously mentioned exercise. I will hop, stabilize, and reach back 
to the starting position, then hop back to the starting position and reach to the targeted position.

The third exercise will be an unanticipated hop to stabilization with me standing in the middle of a nine-marker grid. A randomized, sequence of numbers will be displayed on a computer screen in front of me. Each number will correspond to a hop target position. As the progression of numbers change, I will hop to the new target position. I will do three repetitions.

The fourth and final exercise is the single-limb stance. I will perform three repetitions of a single-limb stance. The exercise will be made more difficult by the environment and conditions, such as eyes open, eyes closed, standing on foam, and throwing a ball.

The four-week dynamic balancing-training program has several levels of difficulty. I will be progressed accordingly. At the end of the fourth week FADI and FADI Sport, VAS, ROM, US, and SEBT measurements will be taken again. I will now complete the study and will be released from this study.

\section{Risks and Discomforts}

I understand there are no known or expected risks for me from participation in this study. I understand that I will be exposed to a stress X-ray by a board certified orthopedist and ultrasonography by a board certified ultrasonographer in this study. I understand mild muscle soreness in my injured lower extremity may occur with performing the GISTM treatment. The treatment will only occur every other day. Dynamic balancing-training program exercises will be the only known or expected discomforts with performing the GISTM and dynamic balancing-training program. I understand that I will be doing dynamic stretching before treatment for the dynamic balancing-training program to reduce muscle soreness, but should be minimal at worst. After completion of the dynamic balancing-training program ice will be applied to the injured extremity to also aid in prevention of muscle soreness. While performing any testing, or exercise I understand that I will not lose their balance because they will be performing the test with their eyes opened. However, I understand that I will be instructed to touch down with the non-injured foot during single-limb balancing exercises if I feel I'm losing my balance.

I understand that every precaution has been taken to prevent me from being injured in this study. If an adverse physical or psychological reaction were to occur during any point of the study, appropriate care or referral will be made available. Should any injury occur to me, I understand that Jessica L. Schaefer, BS, ATC will provide first aid and make any necessary medical referrals at my expense.

\section{Alternative}

I do not have to participate in this study. No negative actions will be taken against me if I choose not to participate. At the present time there are no other alternatives to consider. 


\section{Benefits}

I may not receive any direct benefit from this study. The knowledge gained from this study may eventually benefit others.

\section{Financial Considerations}

I understand that I will not receive any monetary compensation for participation in this study.

\section{Confidentiality}

Any information about me that is obtained as a result of participation in this research will be kept as confidential as legally possible. My research records and test results, just like hospital records, may be subpoenaed by court order or may be inspected by federal regulatory authorities without my additional consent. In addition, there are certain instances where the researcher is legally required to give information to the appropriate authorities. These would include mandatory reporting of infectious diseases, mandatory report of information about behavior that is imminently dangerous to me such as suicide, child abuse, etc. In any publications that result form this research neither my name nor any information, which I might be identified, will be published without my consent.

\section{Voluntary Participation}

Participation in this study is voluntary. You may refuse to participate in this study. You may withdraw from this study at any time. Refusal to participate or withdraw will not affect your child's future care, or your class standing or grades, as appropriate and will involve no penalty to you or your position on the team. In the event new information becomes available that may affect your willingness to participate in this study this information will be given to you so that you can make an informed decision about whether or not to continue participation. You have been given the opportunity to ask questions about the research, and have received answers concerning areas you did not understand.

Upon signing this form I will receive a copy.

I willingly consent to participate in this research.

$\overline{\text { Signature of Subject }} \overline{\text { Printed Name }} \overline{\text { Date/Time }}$

The parent/guardian has had the opportunity to have questions addressed. The parent/guardian willingly agrees to allow his/her child to be in the study.

$\overline{\text { Signature of Co-Investigator }} \overline{\text { Printed Name }} \overline{\text { Date/Time }}$


THE EFFECTS OF A RANDOMIZED FOUR-WEEK GRASTON INSTRUMENTEDASSISTED SOFT TISSUE MOBLIZATION DYNAMIC BALANCING-TRAINING PROGRAM ON INDIVIDUALS WITH CHRONIC ANKLE INSTABILITY.

\section{Co-Investigator(s);}

"Jessica L. Schaefer, BS, ATC"

\section{Contact Persons}

In the event you experience any side effects or injury related to his research, you should contact Jessica Lynn Schaefer, ATC at (812) 686-0973 or at jschaef3@mix.wvu.edu. If you have any question, concerns, or complaints about this research, you can contact Dr. Michelle A. Sandrey, PhD, ATC at (304) 293- 3295 Ext. 5220 or at msandrey@mail.wvu.edu.

For information regarding my rights as a research subject, I may contact the Executive Secretary of the Review Board at (304) 293-7073.

\section{Introduction}

You have been asked to participate in this study, which has been explained to me by Jessica Lynn Schaefer, BS, ATC. This study is being conducted by the Principle Investigator, Michelle A Sandrey, $\mathrm{PhD}$, ATC and the Co-Investigator, Jessica L. Schaefer, BS, ATC in the College of Physical Activities and Sport Sciences at West Virginia University. This research is being conducted to fulfill the requirements for a thesis in Athletic Training in the College of Physical Activities and Sport Sciences at West Virginia University under the supervision of Michelle A. Sandrey, PhD, ATC.

\section{Purpose of Study}

The purpose of this study aims to investigate the affects of Graston InstrumentedAssisted Soft Tissue Mobilization (GISTM) technique and its influence on improving range of motion, neovascularization, collagen alignment, pain and disability in individuals experiencing functional ankle instability following a four-week dynamic balancing-training program.

\section{Description of Procedures}

\section{Procedures}

This study will be conducted at University High School, 991 Price Street, Morgantown, WV 26505 or West Virginia University Athletic Training Research Lab, PO Box 6116, Morgantown, WV 26505. All testing and training procedures will be done in the athletic training room and gymnasium so that subjects may hear and understand all directions in a quiet atmosphere that is free from any distractions. 
At an orientation meeting the purpose of this study will be explained to me. I will be given a demographic questionnaire (gender, height, past and current medical history) and have special tests done for inclusion/exclusion into the study. This will take approximately twenty minutes to complete. I do not have to answer all the questions. I will have the opportunity to see the questionnaire before singing this assent and Authorization to Use or Disclose Protected Health Information forms. All complete forms will be confidential. If I am an eligible subject, I will be contacted by the primary researcher and will schedule a meeting time for approximately one hour for further testing using the Foot and Ankle Ability Measures (FAAM) and FAAM Sport, Visual Analog Scale (VAS), range of motion (ROM), Ultrasonography (US), and Star Excursion Balance Test (SEBT). I will then be instructed on the tests that I will be asked to perform.

I will have a one in three chance of being randomly assigned to either one of the treatment/balancing-training groups or the control group. I will then be asked for my full cooperation and to work to the best of my ability two times a week for forty-five minutes over a four-week period. I will complete a final sixty-minute post-testing session for the FAAM and FAAM Sport, VAS, ROM, US, and SEBT, which will conclude my participation. The post-test will be performed to the exact specifications as the pre-test, and will be completed within the week following the final treatment/balancing-training session of the fourth week.

After my level of functional ankle instability is determined by the demographic questionnaire. The special tests will be the final inclusion or exclusion criteria into the study. If I have a grade III sprain determined by a board certified orthopedic surgeon I will be excluded from the study.

I will be asked to answer the FAAM-26 item questionnaire with one response that will most clearly describe my condition within the past week. Upon completion, I will answer the FAAM Sport 8-item questionnaire with one response that will most clearly describe my condition within the past week.

I will then be asked to make a mark on the $10 \mathrm{~cm}$ line that will accurately describe my pain level at that instant.

My ankle range of motion will be taken. I will be instructed to sit, with my knee off the edge of the table for the first two measurements. The primary researcher will instruct me to move my ankle up and down as far as possible. I will then be asked to lay face down on the table with my ankle off the end of the table. I will be instructed to move my foot in and out as far as possible.

I will next have ultrasonography performed on my ankle over the anterior talofibular ligament (ATF) and the calcaneofibular ligament (CF). I will be instructed to lie down on the table with my knee straight and ankle pointing down and in to image the ATF ligament. Next, I will be instructed to move my ankle up and in to image the CF ligament. The images of my ankle ligaments will be stored and saved under my 
identification number that will be randomly assigned to keep records confidential.

My true leg length will be measured to normalize data. Instructions will be given to me to stand in the middle of a grid formed by three lines extending out at $135^{\circ}$ from each other. I will be asked to reach as far as possible along the three lines [anterior (A), posteromedial (PM), and posterolateral (PL)] lines, make a light touch on the line, and return the reaching leg back to the center, while maintaining a single-leg stance with the other leg in the center of the grid.

I will be allowed to practice reaching in each of the three directions three times. Following a 5-min rest period, I will perform three trials in each of the three directions. The reach distance will be recorded from the center of the grid to point of maximum distance by my reach leg.

Interventions

I will perform a ten-minute dynamic Flex Band flexibility warm-up routine regardless of the experimental or control group I will be randomly assigned. The dynamic warm-up will consist of ankle, calf, achilles, hamstring, groin, I-T Band, and concludes with quadriceps/hip flexor stretches on both legs.

If I am randomly assigned to the Graston Instrumented-Assisted Soft Tissue Mobilization (GISTM) experimental group or GISTM-Sham experimental group, I will first receive an eight to ten minute treatment. I will be instructed to lay face done with my feet over the edge of the table. An emollient will be rubbed onto my leg to prevent friction. The primary researcher will then take the tools and gently move up and down my leg. After the bottom of my foot and calf have been worked with the tools, I will be instructed to lie on the table face up. Again emollient will be rubbed onto my leg. The primary researcher will again move up and down my leg and foot. Finally, I will be instructed to lay on my side with a pillow between my legs for support, again emollient will be applied to my leg. After the GISTM treatment the tools will be cleaned with SaniCide, a hard surface disinfectant. The primary researcher will now take the tools and work around my ankle. Once my treatment is completed or if I am in the control experimental group, the primary researcher will instruct me in the dynamic balancing-training program.

The dynamic balancing-training program is a four-week progressive program for those with chronic ankle instability. I will be shown a video that demonstrates the exercises in their entirety. I will perform single-limb hops to stabilization, hop to stabilization and reach, unanticipated hop to stabilization, and single-limb stance.

The first exercise will require me to hop in four directions ten times. Each repetition will consist of a hop from the starting position to the target position at 18, 27, or 36 inches. After I stabilize my balance in a single-limb stance, I will hop in the exact opposite direction back to the starting position and stabilize in the single-limb stance.

The second exercise is a five-repetition hop to stabilization and reach exercise and will be a combination of the previously mentioned exercise. I will hop, stabilize, and reach back 
to the starting position, then hop back to the starting position and reach to the targeted position.

The third exercise, will be an unanticipated hop to stabilization with me standing in the middle of a nine-marker grid. A randomized, sequence of numbers will be displayed on a computer screen in front of me. Each number will correspond to a hop target position. As the progression of numbers change, I will hop to the new target position. I will do three repetitions.

The fourth and final exercise is the single-limb stance. I will perform three repetitions of a single-limb stance. The exercise will be made more difficult by the environment and conditions, such as eyes open, eyes closed, standing on foam, and throwing a ball.

The four-week dynamic balancing-training program has several levels of difficulty. I will be progressed accordingly. At the end of the fourth week FADI and FADI Sport, VAS, ROM, US, and SEBT measurements will be taken again. I will now complete the study and will be released from this study.

\section{Risks and Discomforts}

I understand there are no known or expected risks for me from participation in this study. I understand that I will be exposed to a stress X-ray by a board certified orthopedist and ultrasonography by a board certified ultrasonographer in this study. I understand mild muscle soreness in my injured lower extremity may occur with performing the GISTM treatment. The treatment will only occur every other day. Dynamic balancing-training program exercises will be the only known or expected discomforts with performing the GISTM and dynamic balancing-training program. I understand that I will be doing dynamic stretching before treatment for the dynamic balancing-training program to reduce muscle soreness, but should be minimal at worst. After completion of the dynamic balancing-training program ice will be applied to the injured extremity to also aid in prevention of muscle soreness. While performing any testing, or exercise I understand that I will not lose their balance because they will be performing the test with their eyes opened. However, I understand that I will be instructed to touch down with the non-injured foot during single-limb balancing exercises if I feel I'm losing my balance.

I understand that every precaution has been taken to prevent me from being injured in this study. If an adverse physical or psychological reaction were to occur during any point of the study, appropriate care or referral will be made available. Should any injury occur to me, I understand that Jessica L. Schaefer, BS, ATC will provide first aid and make any necessary medical referrals at my expense.

\section{Alternative}

I do not have to participate in this study. No negative actions will be taken against me if I choose not to participate. At the present time there are no other alternatives to consider. 


\section{Benefits}

I may not receive any direct benefit from this study. The knowledge gained from this study may eventually benefit others.

\section{Financial Considerations}

I understand that I will not receive any monetary compensation for participation in this study.

\section{Confidentiality}

Any information about me that is obtained as a result of participation in this research will be kept as confidential as legally possible. My research records and test results, just like hospital records, may be subpoenaed by court order or may be inspected by federal regulatory authorities without your additional consent. In addition, there are certain instances where the researcher is legally required to give information to the appropriate authorities. These would include mandatory reporting of infectious diseases, mandatory report of information about behavior that is imminently dangerous to me such as suicide, child abuse, etc. In any publications that result form this research neither my name nor any information form, which I might be identified, will be published without my consent.

\section{Voluntary Participation}

Participation in this study is voluntary. I may refuse to participate in this study. I may withdraw from this study at any time. Refusal to participate or withdraw will not affect my future care, or my class standing or grades, as appropriate and will involve no penalty to me or my position on the team. In the event new information becomes available that may affect your willingness to allow me to participate in this study this information will be given to me so that you can make an informed decision about whether or not to continue my participation. I have been given the opportunity to ask questions about the research, and have received answers concerning areas you did not understand.

Upon signing this form I will receive a copy.

I willingly consent to participate in this research.

$\begin{array}{lll}\overline{\text { Signature of Subject }} & \overline{\text { Date/Time }} \\ \overline{\text { Signature of Co-Investigator }} & & \\ \text { Date/Time }\end{array}$


Table C4. Authorization to Use or Disclose Protected Health Information

\section{Authorization to Use or Disclose Protected Health Information}

(PHI)

\section{West Virginia University}

I hereby voluntarily authorize the use or disclosure of my individually identifiable health information as described below.

Patient Name:

ID Number:

Date of Birth: IRB Protocol \#:

Persons/organizations providing the protected health information (e.g. hospitals): Individual subjects affiliated with University High School, West Virginia University, local Morgantown and WVU physicians.

Persons/organizations receiving the information (e.g. investigators, clinical coordinators, sponsor, FDA):

Jessica Lynn Schaefer ATC

Michelle A. Sandrey, PhD, ATC

The following information well be used:

A self-report injury history questionnaire (lower extremity injuries within the past three months, surgery to the lower extremity, fractures or breaks to the lower extremity).

The information is being disclosed for the following purposes (Start with the Title of the study and include additional information (e.g. screening and recruiting subjects; analyzing research data, or other specified purposes):

The effects of a randomized four-week Graston Instrumented-Assisted Soft Tissue Mobilization and dynamic balancing-training program on individuals with functional ankle instability. Information will be used to screen subjects for inclusion and exclusion criteria of the study.

I may revoke this authorization at any time notifying the Co-Investigator in writing at:

Jessica Lynn Schaefer ATC

School of Physical Education

P.O. Box 6116

Morgantown, WV 26505

If I do revoke my authorization, any information previously disclosed cannot be withdrawn. Once information about me is disclosed in accordance with this authorization, the recipient may redisclose it and the information may no longer be protected by federal privacy regulations. 
I may refuse to sign this authorization form. My clinical treatment may not be affected by whether or not I sign this form. I may not be allowed to participate in the research if I do not sign the form.

This authorization will expire on the date that the research study ends. (Other options for expiration include an actual date of expiration, occurrence of a particular event, or "none" if the authorization will have no expiration date.)

Expiration date:

I will be given a copy of this authorization form.

Signature of subject or subject's legal representative

Date

(Form MUST be completed before signing)

\section{Printed name of subject's legal representative}

Relationship to the subject (Initial beside):

Parent

Medical power of attorney/representative

Legal guardian

Health care surrogate 


\section{Table C5. Demographics ${ }^{10,53}$}

\section{Instructions}

This form will be used to categorize your ankle instability. A separate form should be used for the right and left ankles. Please fill out the form completely. If you have any question, please ask the administrator of the survey. Thank you for your participation.

Male / Female

Height:

\section{Ankle (circle): Right or Left}

1. Have you ever sprained an ankle?

If yes, did the initial episode involve your ankle "rolling inward"?

If no, do not continue to fill out this questionnaire.

2. Have you ever seen a doctor for an ankle sprain?

If yes, How did the doctor categorize your most serious ankle sprain?
Mild (grade 1)
Moderate (grade II)
Severe (grade III)

3. Did you ever use a devise (such as crutches) because you could not bear weight due to an ankle sprain?

If yes, In the most serious case, how long did you need to use the device?
1-3 days
4-7 days
$1-2$ weeks
2-3 weeks

4. Did the injury to your ankle require immobilization of any form (cast, braces, tape, etc)?

5. Have you been walking around unassisted without a "limp," for at least the past 3 months?

6. Have you had any fractures (breaks) in either of your ankles?

7. Have you had any surgeries in either of your lower extremities?

8. Is the injured/unstable ankle functionally weaker, more painful, "looser," and less functional than your uninvolved ankle?

9. Have you ever experienced a sensation of your ankle "giving way"?

If yes, When was the last time your ankle "gave way"?
$<3$ weeks
3-6 weeks
1-3 months
4-7 months
$>1$ year

10. Does your ankle ever feel unstable while walking on a flat surface?

11. Does your ankle ever feel unstable while walking on uneven ground?

12. Does your ankle ever feel unstable during recreational or sport activity?

13. Does your ankle ever feel unstable while going up stairs?

14. Do you attribute your current instability to past injuries to the affected ankle?

15. Are you currently involved in a "formal" rehabilitation program for the affected ankle?

Date

Signature

Administrator Use ONLY

Participant ID \# 


\section{Foot and Ankle Ability Measure (FAAM)}

Please answer every question with one response that most closely describes to your condition within the past week.

If the activity in question is limited by something other than your foot or ankle mark not applicable (N/A).

\begin{tabular}{|c|c|c|c|c|c|}
\hline & $\begin{array}{c}\text { No } \\
\text { difficulty }\end{array}$ & $\begin{array}{c}\text { Slight } \\
\text { difficulty }\end{array}$ & $\begin{array}{l}\text { Moderate } \\
\text { difficulty }\end{array}$ & $\begin{array}{l}\text { Extreme } \\
\text { difficulty }\end{array}$ & $\begin{array}{l}\text { Unable } \\
\text { to do }\end{array}$ \\
\hline Standing & $\square$ & $\square$ & $\square$ & $\square$ & $\square$ \\
\hline Walking on even ground & $\square$ & $\square$ & $\square$ & $\square$ & $\square$ \\
\hline $\begin{array}{l}\text { Walking on even ground } \\
\text { without shoes }\end{array}$ & $\square$ & $\square$ & $\square$ & $\square$ & $\square$ \\
\hline Walking up hills & $\square$ & $\square$ & $\square$ & $\square$ & $\square$ \\
\hline Walking down hills & $\square$ & $\square$ & $\square$ & $\square$ & $\square$ \\
\hline Going up stairs & $\square$ & $\square$ & $\square$ & $\square$ & $\square$ \\
\hline Going down stairs & $\square$ & $\square$ & $\square$ & $\square$ & $\square$ \\
\hline Walking on uneven ground & $\square$ & $\square$ & $\square$ & $\square$ & $\square$ \\
\hline Stepping up and down curbs & $\square$ & $\square$ & $\square$ & $\square$ & $\square$ \\
\hline Squatting & $\square$ & $\square$ & $\square$ & $\square$ & $\square$ \\
\hline Coming up on your toes & $\square$ & $\square$ & $\square$ & $\square$ & $\square$ \\
\hline Walking initially & $\square$ & $\square$ & $\square$ & $\square$ & $\square$ \\
\hline \multicolumn{6}{|l|}{ Walking 5 minutes or less } \\
\hline $\begin{array}{l}\text { Walking approximately } 10 \\
\text { minutes }\end{array}$ & $\square$ & $\square$ & $\square$ & $\square$ & $\square$ \\
\hline $\begin{array}{l}\text { Walking } 15 \text { minutes or } \\
\text { greater }\end{array}$ & $\square$ & $\square$ & $\square$ & $\square$ & $\square$ \\
\hline
\end{tabular}


Because of your foot and ankle how much difficulty do you have with:

$\begin{array}{lcccccc} & \begin{array}{l}\text { No } \\ \text { difficulty } \\ \text { at all }\end{array} & \begin{array}{c}\text { Slight } \\ \text { difficulty }\end{array} & \begin{array}{c}\text { Moderate } \\ \text { difficulty }\end{array} & \begin{array}{c}\text { Extreme } \\ \text { difficulty }\end{array} & \begin{array}{c}\text { Unable } \\ \text { to do }\end{array} & \text { N/A } \\ \text { Home Responsibilities } & \square & \square & \square & \square & \square & \square \\ \text { Activities of daily living } & \square & \square & \square & \square & \square \\ \text { Personal care } & \square & \square & \square & \square & \square & \square \\ \begin{array}{l}\text { Light to moderate work } \\ \text { (standing, walking) }\end{array} & \square & \square & \square & \square & \square & \square \\ \begin{array}{l}\text { Heavy work (push/pulling, } \\ \text { climbing, carrying) }\end{array} & \square & \square & \square & \square & \square & \square \\ \text { Recreational activities } & \square & \square & \square & \square & \square\end{array}$

How would you rate your current level of function during your usual activities of daily living from 0 to 100 with 100 being your level of function prior to your foot or ankle problem and 0 being the inability to perform any of your usual daily activities?

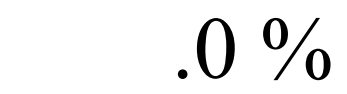


FAAM Sports Scale

Because of your foot and ankle how much difficulty do you have with:

\begin{tabular}{|c|c|c|c|c|c|c|}
\hline & $\begin{array}{l}\text { No } \\
\text { difficulty } \\
\text { at all }\end{array}$ & $\begin{array}{c}\text { Slight } \\
\text { difficulty }\end{array}$ & $\begin{array}{l}\text { Moderate } \\
\text { difficulty }\end{array}$ & $\begin{array}{l}\text { Extreme } \\
\text { difficulty }\end{array}$ & $\begin{array}{l}\text { Unable } \\
\text { to do }\end{array}$ & $\mathrm{N} / \mathrm{A}$ \\
\hline Running & $\square$ & $\square$ & $\square$ & $\square$ & $\square$ & $\square$ \\
\hline Jumping & $\square$ & $\square$ & $\square$ & $\square$ & $\square$ & $\square$ \\
\hline Landing & $\square$ & $\square$ & $\square$ & $\square$ & $\square$ & $\square$ \\
\hline $\begin{array}{l}\text { Starting and stopping } \\
\text { quickly }\end{array}$ & $\square$ & $\square$ & $\square$ & $\square$ & $\square$ & $\square$ \\
\hline Cutting/lateral movements & $\square$ & $\square$ & $\square$ & $\square$ & $\square$ & $\square$ \\
\hline Low impact activities & $\square$ & $\square$ & $\square$ & $\square$ & $\square$ & $\square$ \\
\hline $\begin{array}{l}\text { Ability to perform activity } \\
\text { with your normal technique }\end{array}$ & $\square$ & $\square$ & $\square$ & $\square$ & $\square$ & $\square$ \\
\hline $\begin{array}{l}\text { Ability to participate in your } \\
\text { desired sport as long as you } \\
\text { would like }\end{array}$ & $\square$ & $\square$ & $\square$ & $\square$ & $\square$ & $\square$ \\
\hline
\end{tabular}

How would you rate your current level of function during your sports related activities from 0 to 100 with 100 being your level of function prior to your foot or ankle problem and 0 being the inability to perform any of your usual daily activities?

$\square \square \square .0 \%$

Overall, how would you rate your current level of function?

Normal

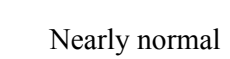

Abnormal

Severely abnormal 
1. Subjects' identification number was placed in the top right corner.

2. Subject marked pain level at that instant on the $10 \mathrm{~cm}$ line.

3. The primary researcher measured the mark going from left to right in millimeters $(\mathrm{mm})$.

4. The mean was found between the pre- and post-test measurements.

Visual Analog Scale (VAS)

How severe is your pain today? Place a vertical mark on the line below to indicate how bad you feel your pain is today. 
Table C8. Range of Motion: Goniometry

1. Ankle range of motion was measured in degrees using a goniometer.

2. Subjects' were instructed to sit, with the knee flexed off the end of a treatment table.

3. The subject was asked to plantarflex the ankle as far as possible. The goniometer was placed 1) with the stationary arm along the midline of the fibula, fibula head to lateral malleolus; and 2) the movable arm along the midline of the fifth metatarsal.

4. The subject was asked to dorsiflex the ankle as far as possible. The goniometer was placed 1) with the stationary arm along the midline of the fibula, fibular head to lateral malleolus; and 2) the movable arm along the midline of the fifth metatarsal.

5. Subjects were instructed to lay prone.

6. Primary researcher then stabilized the tibia in the sagittal plane.

7. Subjects were instructed to invert the calcaneus as far possible. The goniometer was placed 1) with the stationary arm in alignment with the midline of the leg; and 2) the movable arm aligned with the midline of the calcaneus.

8. Subjects were instructed to evert the calcaneus as far as possible. The goniometer was placed 1) with the stationary arm in alignment with the midline of the leg; and 2) the movable arm aligned with the midline of the calcaneus. 
1. Subjects' identification number was entered into the computer for subject's folder of images.

2. Subjects were instructed to lie in the supine position, knee straight, with the ankle plantarflexed and inverted on the treatment table.

3. The probe was placed in a slightly oblique position from the malleolus toward the forefoot to identify the ATF ligament.

4. The image of the ATF ligament was stilled and saved in the subjects' folder.

5. The color doppler/power doppler was turned on and an image were stilled and saved in the subjects' folder.

6. Subjects were instructed to lie in the supine position, knee straight, with ankle dorsiflexed and inverted on the treatment table.

7. The probe was placed in a slightly posterior oblique direction toward the calcaneus to identify the CF ligament.

8. The image of the CF ligament was stilled and saved in his/her subject folder.

9. The color doppler/power doppler was turned on and then an image was stilled and saved in the subjects' folder. 
Figure C1. Star Excursion Balance Test (SEBT)

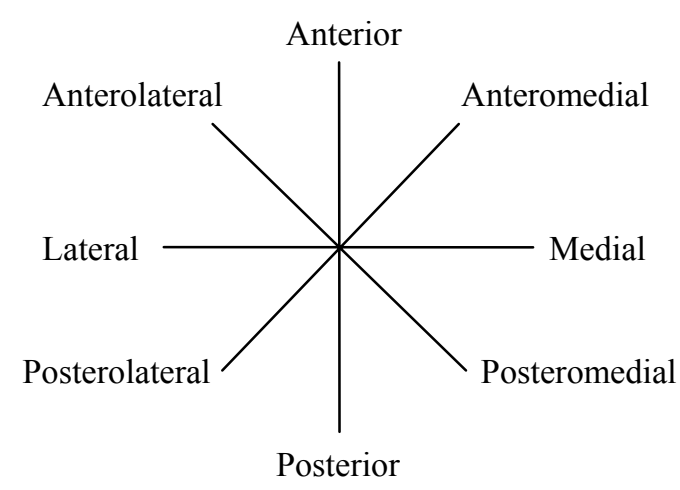

Left-Leg Stance

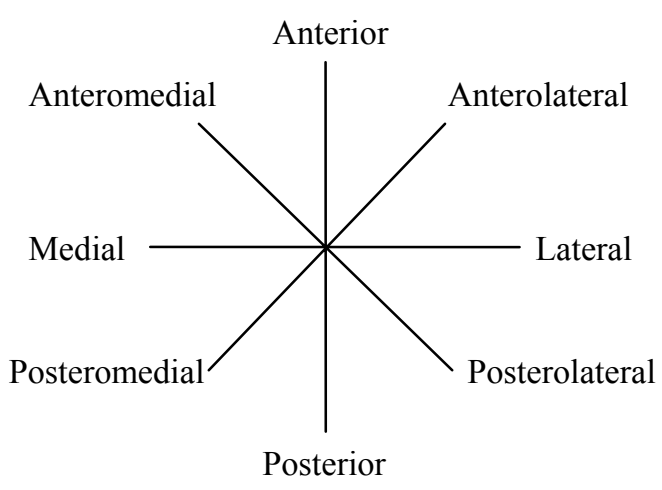

Right-Leg Stance

1. Subjects' leg length was measured in centimeters and recorded to normalize distances to his/her height.

2. Warm-up session of five minutes on a stationary bicycle at 120 revolutions per minute.

3. The subjects took a five-minute break after the practice trials.

4. Subjects selected an index card to randomly determine in which direction they started.

5. Subjects were instructed to stand in the center of the star grid and maintain a singleleg stance while reaching with the opposite leg to touch as far as possible along the randomly chosen reach direction.

6. Subjects were instructed to touch the farthest point possible as light as possible along a chosen reach direction with the most distal part of their reach foot.

7. Subjects whose reaching leg was the right went around the star grid in a counterclockwise fashion while subjects whose reaching leg was the left went around the star grid in a clockwise fashion.

8. Subjects were instructed to perform three practice trials in each of the three directions with a 15 -second rest between each excursion.

9. After a five-minute rest following the last practice trial, testing began.

10. Three trials were performed in each of the three excursions (anterior, posteromedial, and posterolateral) with a 15 -second rest between each reach. 
11. Trials were discarded and repeated if the reach foot was used to provide considerable support when touching the ground, if the subjects' stance foot was lifted from the center of the star grid, or if the subjects were not able to maintain their balance at any point in the trial.

12. The average excursions for each reach direction will be calculated and recorded.

13. The average excursions for the three-reach direction was divided by leg length and multiplied by 100 to determine each subject's dynamic balance score in each of the three reach directions. 
Figure C2. Dynamic Flex Band Flexibility Warm-Up Routine ${ }^{82}$
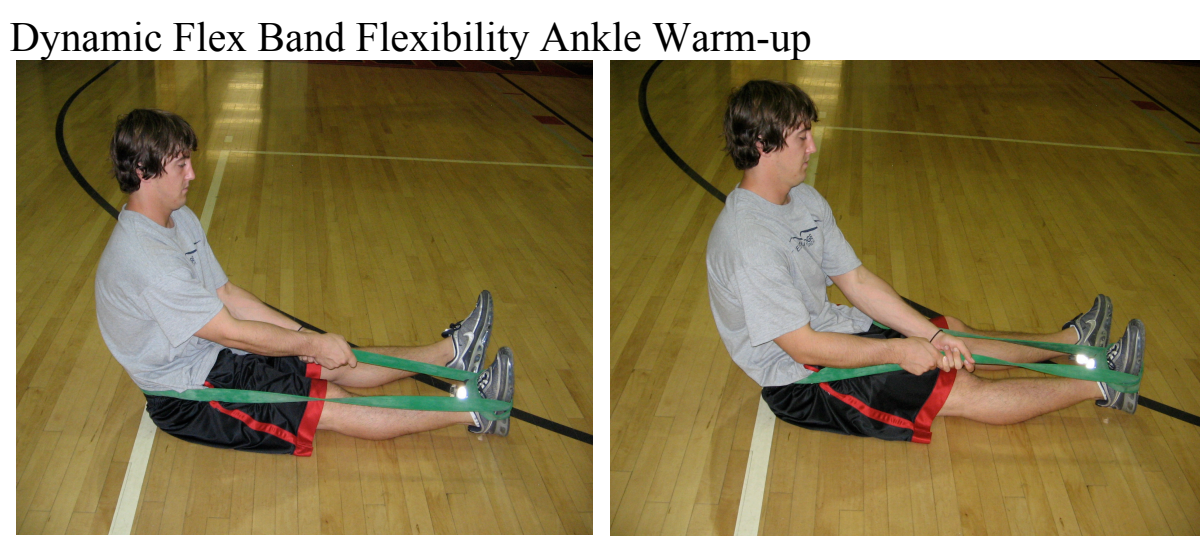

1. Ankles (Inversion/Eversion)

a. Sit on the floor, position the band around the back at the belt-line.

b. Wrap the end of the band around the arch of the foot, forming a loop.

c. Hold onto the inner side of the band with both hands, turn the ankle in and out as far as it will go 10 times.

d. Then grasp the outer side of the band and turn the ankle out and then in 10 times.

Dynamic Flex Band Gastrocnemius Warm-up

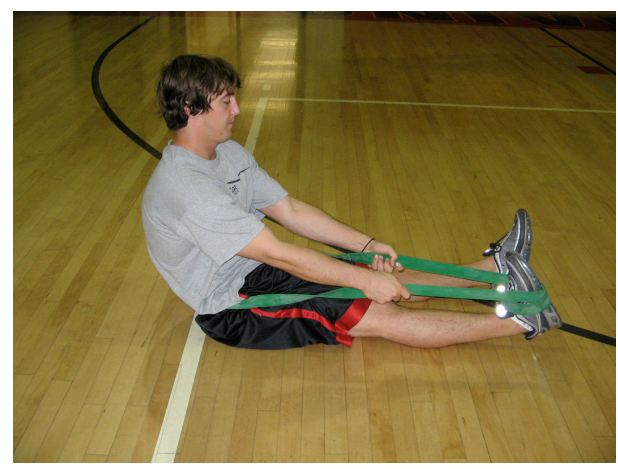

2. Gastrocnemius Stretch

a. Reach down with both hands and adjust the loops so that it is around the ball of the foot.

b. With one hand on each side of the band below the knee, stretch the gastrocnemius by pulling toward the body with the band (dorsiflexion).

c. Then, point the toes away from the body (plantarflexion).

d. Pull back and press out 10 times. 


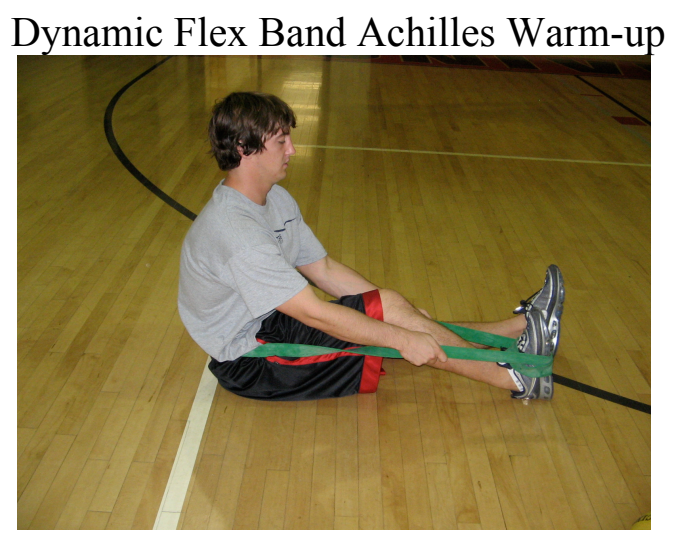

3. Achilles Stretch

a. With the band still positioned around the ball of the foot, plant the heel on the floor close to the body.

b. Stretch out the Achilles by pulling the band toward the body.

c. Hold the band against the knee for added resistance and press the toes toward the floor.

d. Repeat 10 times.

4. At this point, grasp the "loop" in both hands and transfer it to the other foot. Repeat the Inversion/Eversion, Gastronemius and Achilles Stretches for the other ankle before proceeding.

5. After completing the Ankle Work for both legs, remove band from around the waist.

\section{Dynamic Flex Band Hamstring Warm-up}

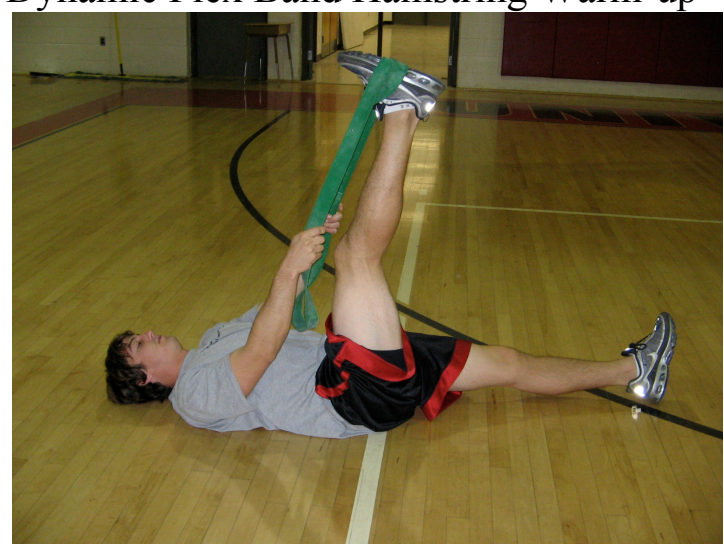

6. Hamstring Stretch

a. Re-wrap the band around the arch of the foot.

b. Lie back on the floor and stretch the hamstring as far as it will go by "walking" the hands up the band.

c. As the leg returns to the floor, let the hands slide down the band while providing resistance

d. Repeat 5 times.

e. Then "walk" up the band to the top and lock out the knee. 
f. Hold that position for 5 seconds.

g. Next, give a slight bend to the knee and lock it out again, keeping pressure on the Golgi tendons behind the knee.

h. Bend and lock out 5 times.

i. Then, stretch back and hold again for 5 seconds.

\section{Dynamic Flex Band Groin Warm-up}

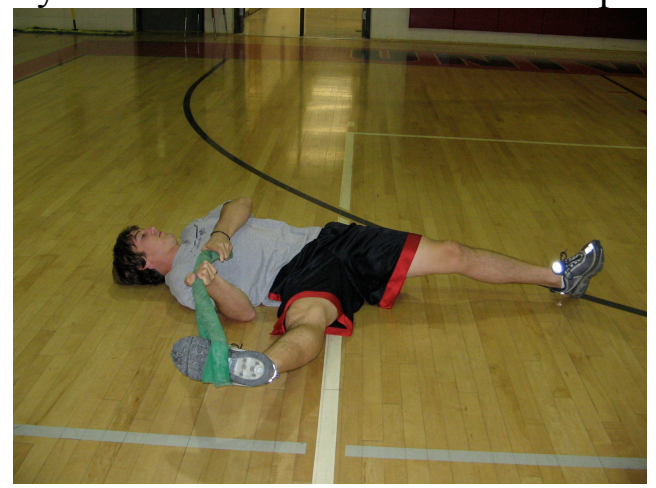

7. Groin Stretch

a. With the loop of the band still around the arch of the foot, grab the end of the band in the opposite hand.

b. With the other hand, grab the middle of the band and take the band behind the head, positioning the elbow on the floor and the leg out to the side as far as it will go.

c. Bend the knee slightly and then lock it out.

d. Repeat 10 times.

Dynamic Flex Band IT-Band Warm-up

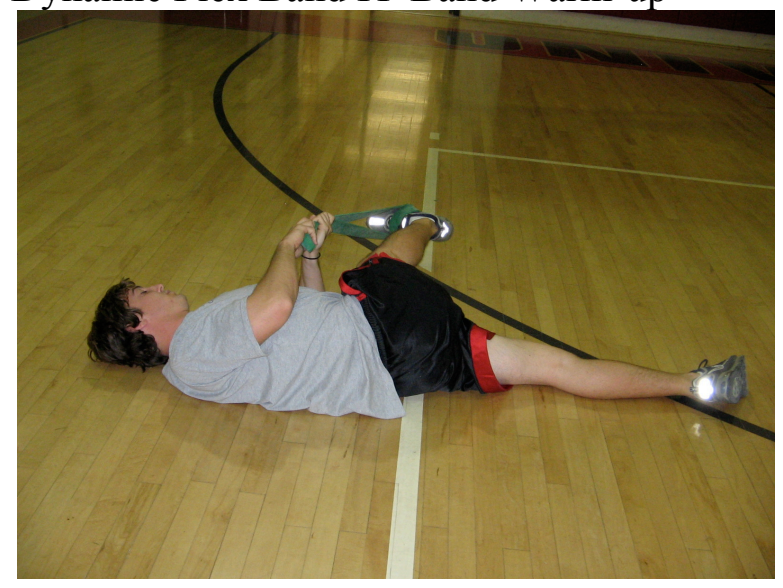

8. I-T Band Stretch

a. With the band still positioned around the arch of the foot, grab the end of the band with the same hand.

b. With the opposite hand, grab the middle of the band and take the band behind the head. 
c. Set the elbow down on the floor and read the leg across the body, keeping both shoulders on the ground.

d. Lock the knee and hold for 5 seconds.

e. Next, bend the knee slightly and straighten 10 times.

f. Add more pressure by pulling on the band and hold for 5 seconds.

\section{Dynamic Flex Band Quadriceps/Hip Flexor Warm-up}

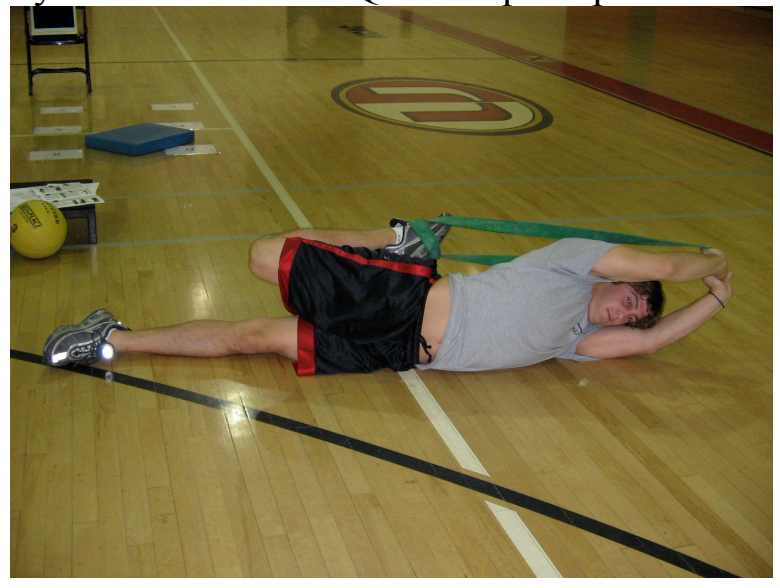

9. Quadriceps \& Hip Flexor Stretch

a. With the band still positioned around the arch of the foot, roll onto the opposite side with the knees bent.

b. Holding the top of the band above the head with both hands, move the leg around to stretch out the hip area.

c. Move the leg in any direction for about 15-30 seconds.

10. Now, sit up and transfer the band to the other foot. Repeat the Hamstring, Groin, I-T Band, and Quadriceps/Hip Flexor Stretches for the other leg. 
Figure C3. Dynamic Balance-Training Program ${ }^{35}$

Hop to Stabilization

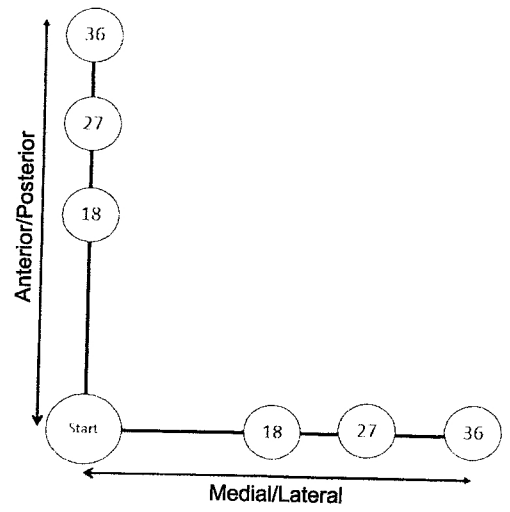

Hop to Stabilization

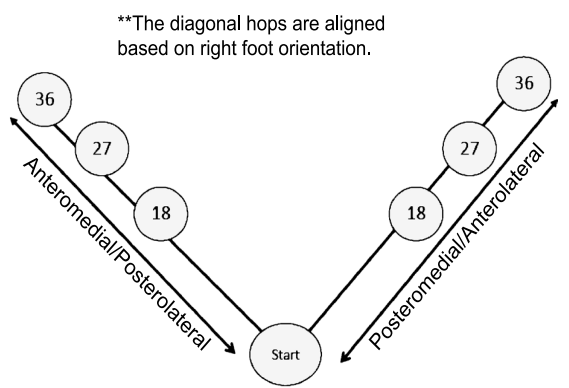

1. Single-limb Hops to Stabilization (10 Repetitions)

a. The subjects performed ten hops in each of the four directions, anterior/posterior, medial/lateral, anterolateral/posteromedial, and anteromedial/posterolateral.

b. Each repetition consisted of a hop from the starting position to the target position (18, 27, or 36 inches).

c. After stabilizing balance in a single-limb stance, subjects hopped in the exact opposite direction back to the starting position and stabilized in the single-limb stance.

2. Hop to Stabilization and Reach (5 repetitions)

a. A combination of the previously mentioned exercise.

b. However, after stabilization in the single-limb stance, the subject reached back to the starting position.

c. Repetitions were counted in the same manner. Subjects hopped, stabilized, and reached back to the starting position.

d. Then hop back to the starting position and reach to the targeted position.

7 progression levels for Hop to Stabilization and Hop to Stabilization and Reach:

1. 18-inch hop; were allowed to use arms to aid in stabilizing balance after landing

2. 18-inch hop was with hands on hips while stabilizing balance after landing

3. 27-inch hop; were allowed to use arms to aid in stabilizing balance after landing

4. 27-inch hop was with hands on hips while stabilizing balance after landing

5. 36-inch hop; were allowed to use hands to aid in stabilizing balance after landing

6. 36-inch hop was with hands on hips while stabilizing balance after landing

7. 36-inch hop was from a 6-inch platform. 


\section{Unanticipated Hop}

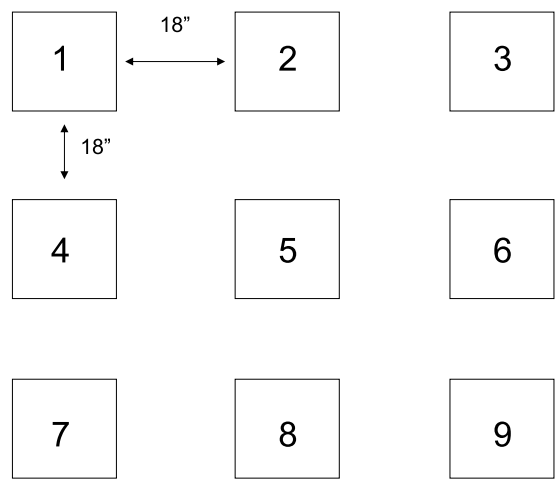

3. Unanticipated Hop to Stabilization (3 sequences of numbers)

a. Subjects stood in the middle of a nine-marker grid (see Figure C11).

b. A randomized, sequence of numbers was displayed on a computer screen in front of the subjects.

c. Each number corresponded to a target position to which the subject hopped.

d. As the progression of numbers changed, subjects hopped to the new target position.

e. The hop to stabilization rules applied for this activity; however, in this case, subjects were allowed to use any combination of hops (AP, ML, $\mathrm{AM} / \mathrm{PL}$, or AL/PM) desired to accomplish the goal of getting through the sequence error-free.

f. As a subject develops proficiency, the amount of time per move was reduced.

g. In each session, subjects performed three sequences of numbers.

The levels of Unanticipated Hop to Stabilization will be as follows:

1. Level 1; five seconds per move

2. Level 2; three seconds per move

3. Level 3; one second per move

4. Level 4; if subject can progress to completion of all moves within one second without error, a foam pad was placed on one of the numbers during the sequence. The subject then continued the progression at the same level of intensity. If the subject cannot complete the course error-free, the time constraint was increased to the level below

5. Level 5; if subject can progress to completion of all moves at level 3 within the foam pad error-free, a step was added to an additional number

6. Level 6; if a subject progresses error-free, an additional foam pad was added to one of the numbers, resulting in two foam pads and one step

7. Level 7; if a subject progresses error-free, an additional step was included, resulting in two foam pads and two steps. 
Single-limb Stance (3 repetitions)

a. Each activity (eyes open and eyes closed) had seven levels of difficulty and is as follows:

b. Single-limb stance with eyes opened:

i. Arms were across chest on hard floor for 60 seconds

ii. Arms were across chest for 30 seconds on foam pad

iii. Arms were across chest for 60 seconds on foam pad

iv. Arms were across chest for 90 seconds on foam pad.

h. With ball toss on foam pad:

i. 30 seconds with arms across chest; 20 throws with 6-lb medicine ball

ii. 60 seconds with arms across chest; 20 throws with 6 -lb medicine ball

iii. 90 seconds with arms across chest; 20 throws with 6 -lb medicine ball.

i. Single-limb stance with eyes closed:

i. Arms were out on hard floor for 30 seconds

ii. Arms were across chest on hard floor for 30 seconds

iii. Arms were across chest on hard floor for 60 seconds

iv. Arms were out on foam pad for 30 seconds

v. Arms were across chest for 30 seconds on foam pad

vi. Arms were across chest for 60 seconds on foam pad

vii. Arms were across chest for 90 seconds on foam pad 
Table C13. Graston Instrumented-Assisted Soft Tissue Mobilization Technique

1. Subjects were instructed to lie prone with his/her foot over the end of the treatment table.

2. Primary researcher was standing.

3. The primary researcher used the GT-4 and GT-5 tool with a two-hand hold sweeping the plantar fascia and heel pad with GT-4. The gastrocnemius-soleus complex was swept and fanned starting proximal to the achilles tendon insertion up to the popliteal fossa using GT-5. Assessing in a neutral position and in dorsiflexion varies the tension of the tissue as it affects the instrument's depth of penetration.

4. The primary researcher used the GT-3 tool with a pencil grip localizing any restrictions within the gastrocnemius-soleus complex, achilles tendon, and plantar fascia.

5. The primary researcher used the GT-2 or GT-3 tool with a two-hand hold placing additional pressure over the knob of GT-2. The soft tissue lying in the groove between the achilles tendon and the fibula was mobilized laterally and medially between the tendon and the tibia.

6. The primary researcher used the GT-2 or GT-3 tool with a one-hand hold. The knob of GT-2 was used to mobilize the plantar fascial strips from the calcaneus to the metatarsal head and back. The fascia tension was varied by flexing and extending the toes.

7. The primary researcher used the GT-2 tool with a one-hand hold. The curve of the tool was used to work between the toes.

8. Subjects were instructed to lie supine with his/her foot over the end of the treatment table.

9. Primary researcher was standing.

10. The primary researcher used GT-5 tool with a two-hand hold scanning the dorsum of the foot by moving proximal to the anterior tibialis.

11. The primary researcher used GT-4 tool, with a two-hand hold, sweeping the dorsum of the foot and anterior tibialis to isolate restrictions. The stroke pattern was reversed for better isolation.

12. The primary researcher depending on size and area used the GT-2 or GT-3 tool with a two-hand hold working around the medial and lateral malleolus. One hand stabilized 
the tool, while the other hand distributed pressure through the knob or curve of GT-2 as needed.

13. The primary researcher used GT-2 or GT-3 tool with a two-hand hold working around the first metatarsal head. One hand stabilized while the other hand mobilized the tool, distributing pressure through the curve of GT-2. Pressure was placed over the knob while scooping between the extensor tendons.

14. The primary researcher used GT-2 or GT-3 tool with a one-hand hold using the GT-2 knob to mobilize the soft tissue around the talocrural joint and distal tibiofibular joint. Ankle dorsiflexion/plantarflexion, inversion/eversion and 1st toe flexion/extension was altered with the other hand. This allowed a different depth of instrument penetration.

15. The primary researcher used GT-2 or GT-3 tool with a two-hand hold sweeping up and down the medial and lateral aspect of the tibia using the knob of GT-2 so as to release fascial compartments attached to this bone. Focus was to isolate restrictions. Initially GT-4 was used for a more general assessment.

16. Subject was instructed to move to a side lying position with a pillow to support the limb.

17. The primary researcher was sitting on a stool at the side of subject.

18. The primary researcher based on size and area used GT-3, GT-4 or GT-2 tool with a pencil grip to strum, fan, sweep, and do J-strokes on the peroneals as needed. 


\section{APPENDIX D}

\section{ADDITIONAL RESULTS}

Table D1. Descriptive Statistics on Means and Standard Deviations for All Dependent Variables.

\begin{tabular}{lllllll}
\hline $\begin{array}{l}\text { Dependent } \\
\text { Variable }\end{array}$ & \multicolumn{2}{c}{ Control/DBT Group } & \multicolumn{2}{c}{ GISTM-S/DBT Group } & \multicolumn{2}{c}{ GISTM/DBT Group } \\
\hline & \multicolumn{2}{c}{ Pre-Test } & \multicolumn{1}{c}{ Post-Test } & Pre-Test & \multicolumn{1}{c}{ Post-Test } & \multicolumn{1}{c}{ Pre-Test } \\
\hline FAAM & $75.909 \pm 7.395$ & $84.000 \pm 0.000$ & $75.333 \pm 7.353$ & $84.000 \pm 0.000$ & $76.231 \pm 6.496$ & $84.000 \pm 0.000$ \\
FAAM & $30.364 \pm 11.201$ & $32.000 \pm 0.000$ & $24.500 \pm 6.215$ & $32.00 \pm 0.000$ & $24.417 \pm 4.259$ & $32.000 \pm 0.000$ \\
Sport & & & & & & \\
VAS & $1.936 \pm 1.854$ & $1.854 \pm 0.000$ & $1.950 \pm 1.544$ & $1.544 \pm 0.432$ & $1.631 \pm 1.291$ & $1.291 \pm 0.161$ \\
ROM & & & & & & \\
DF & $13.273 \pm 2.040$ & $16.091 \pm 3.707$ & $14.000 \pm 3.820$ & $16.167 \pm 3.525$ & $15.269 \pm 2.643$ & $19.269 \pm 2.279$ \\
PF & $28.364 \pm 8.654$ & $29.636 \pm 10.642$ & $25.688 \pm 8.541$ & $30.125 \pm 9.518$ & $26.462 \pm 7.487$ & $33.432 \pm 8.585$ \\
INV & $14.000 \pm 1.414$ & $14.546 \pm 0.757$ & $14.042 \pm 0.916$ & $14.500 \pm 1.128$ & $11.077 \pm 5.086$ & $14.981 \pm 0.069$ \\
EV & $3.136 \pm 1.164$ & $4.364 \pm 0.977$ & $3.250 \pm 1.177$ & $4.708 \pm 0.620$ & $3.808 \pm 1.182$ & $5.000 \pm 0.000$ \\
SEBT & & & & & & \\
A & $70.046 \pm 8.988$ & $75.736 \pm 8.462$ & $70.233 \pm 5.902$ & $75.675 \pm 13.421$ & $70.389 \pm 5.820$ & $83.169 \pm 9.177$ \\
PM & $67.573 \pm 10.171$ & $73.082 \pm 10.923$ & $66.892 \pm 7.664$ & $70.725 \pm 7.884$ & $64.946 \pm 11.388$ & $75.092 \pm 11.133$ \\
PL & $52.618 \pm 12.584$ & $59.718 \pm 11.927$ & $52.400 \pm 6.923$ & $60.683 \pm 14.055$ & $54.485 \pm 9.689$ & $61.746 \pm 13.332$ \\
\hline
\end{tabular}

Key: FAAM=Foot and Ankle Ability Measure; VAS=Visual Analog Scale; ROM=Range of Motion; $\mathrm{DF}=$ Dorsiflexion; $\mathrm{PF}=\mathrm{Plantarflexion}$; INV=Inversion; EV=Eversion; SEBT=Star Excursion Balance Test; $\mathrm{A}=$ Anterior; $\mathrm{PM}=$ Posteromedial; $\mathrm{PL}=$ Posterolateral; GISTM=Graston Instrumented-Assisted Soft Tissue Mobilization.

Table D2. Two-way Repeated Measures ANOVA Results for the FAAM, FAAM Sport, and VAS.

\begin{tabular}{|c|c|c|c|c|c|}
\hline Dependent Variables & $\mathrm{df}$ & $\mathrm{F}$ & $\mathrm{P}$ & ES & $1-\beta$ \\
\hline \multicolumn{6}{|l|}{ FAAM } \\
\hline Time & 1 & 47.963 & $<0.001 *$ & 0.592 & 1.000 \\
\hline Group & 2 & 0.051 & 0.950 & 0.003 & 0.012 \\
\hline Time $x$ Group & 2 & 0.051 & 0.950 & 0.003 & 0.012 \\
\hline \multicolumn{6}{|l|}{ FAAM Sport } \\
\hline Time & 1 & 18.377 & $<0.001^{*}$ & 0.358 & 0.932 \\
\hline Group & 2 & 2.153 & 0.132 & 0.115 & 0.187 \\
\hline Time $x$ Group & 2 & 2.153 & 0.132 & 0.115 & 0.187 \\
\hline \multicolumn{6}{|l|}{ VAS } \\
\hline Time & 1 & 47.475 & $<0.001 *$ & 0.590 & 1.000 \\
\hline Group & 2 & 0.160 & 0.853 & 0.010 & 0.017 \\
\hline Time x Group & 2 & 0.215 & 0.808 & 0.013 & 0.020 \\
\hline
\end{tabular}

Key: FAAM= Foot and Ankle Ability Measure; VAS=Visual Analog Scale; $\mathrm{df}=$ degrees of freedom;

$\mathrm{P}=$ significance; $\mathrm{ES}=$ effect size; 1 - $\beta=$ power; ${ }^{*}=$ Significant

Table D3. Paired Samples t-Test Results for Pre-test and Post-test for FAAM, FAAM Sport, and VAS.

\begin{tabular}{lcc}
\hline \multicolumn{1}{c}{ Dependent Variable } & $\mathrm{t}$ & $\mathrm{P}$ \\
\hline FAAM & -7.130 & $<0.001^{*}$ \\
FAAM Sport & -4.279 & $<0.001^{*}$ \\
VAS & 7.022 & $<0.001^{*}$ \\
\hline
\end{tabular}

Key: FAAM=Foot and Ankle Ability Measure; VAS=Visual Analog; $\mathrm{t}=\mathrm{T}$-test; $\mathrm{P}=$ significance;

$*=$ Significant 
Table D4. Percent Change Scores for FAAM, FAAM Sport, and VAS.

\begin{tabular}{lccc}
\hline Dependent Variable & Control/DBT Group & GISTM-S/DBT Group & GISTM/DBT Group \\
\hline FAAM & 10.7 & $11.5^{*}$ & 10.2 \\
FAAM Sport & 5.3 & 30.6 & $31.1^{*}$ \\
VAS & 4.2 & 20.8 & $20.9^{*}$ \\
\hline
\end{tabular}

Key: FAAM=Foot and Ankle Ability Measure; VAS=Visual Analog Scale; GISTM-S=Graston Instrumented-Assisted Soft Tissue Mobilization-Sham; *=Most percent change by group.

Table D5. Repeated Measures ANOVA Results for ROM.

\begin{tabular}{lccccc}
\hline \multicolumn{1}{c}{ Dependent Variable } & df & F & P & ES & $1-\beta$ \\
\hline ROM & & & & & \\
Time & 1 & 40.087 & $<0.001^{*}$ & 0.548 & 1.000 \\
Group & 2 & 0.498 & 0.612 & 0.029 & 0.036 \\
Time x Group & 2 & 3.720 & 0.035 & 0.184 & 0.379 \\
Movement & 3 & 196.721 & $<0.001^{*}$ & 0.856 & 1.000 \\
Movement x Group & 6 & 0.592 & 0.736 & 0.035 & 0.082 \\
Time x Movement & 3 & 4.331 & 0.045 & 0.116 & 0.275 \\
Time x Movement x Group & 6 & 1.564 & 0.225 & 0.087 & 0.124 \\
\hline
\end{tabular}

Key: ROM=Range of Motion; $\mathrm{df}=$ degrees of freedom; $\mathrm{P}=$ significance; $\mathrm{ES}=$ effect size; 1 - $\beta=$ power;

$*=$ Significant

Table D6. Paired Samples t-Test Results for Pre-test and Post-test for ROM (Mean Differences).

\begin{tabular}{|c|c|c|c|c|c|}
\hline \multirow[t]{2}{*}{ Dependent Variable } & \multirow[t]{2}{*}{$\mathrm{X}$} & \multicolumn{2}{|c|}{$98 \% \mathrm{CI}$} & \multirow[t]{2}{*}{$\mathrm{t}$} & \multirow[t]{2}{*}{$\mathrm{P}$} \\
\hline & & Lower & Upper & & \\
\hline \multicolumn{6}{|l|}{ ROM } \\
\hline DF & -3.028 & -4.098 & -1.957 & -6.895 & $<0.001 *$ \\
\hline $\mathrm{PF}$ & -4.382 & -7.317 & -1.447 & -3.639 & $0.001 *$ \\
\hline IN & -1.729 & -3.154 & -0.304 & -2.958 & $0.006^{*}$ \\
\hline $\mathrm{EV}$ & -1.292 & -1.773 & -0.810 & -6.537 & $<0.001 *$ \\
\hline
\end{tabular}

Key: ROM=Range of Motion; $\mathrm{X}=$ Mean; $\mathrm{SD}=$ Standard Deviation; CI-Confidence Interval;

$\mathrm{DF}=$ Dorsiflexion; $\mathrm{PF}=$ Plantarflexion; $\mathrm{INV}=$ Inversion; $\mathrm{EV}=$ Eversion; $\mathrm{t}=\mathrm{T}$-Test; $\mathrm{P}=$ significance;

$*=$ Significant

Table D7. Pairwise Comparison for ROM (Mean Differences).

\begin{tabular}{lcccc}
\hline Dependent Variable & $\mathrm{X}$ & $\mathrm{P}$ & \multicolumn{2}{c}{$99 \%$ Confidence Interval } \\
\hline & & & Lower Boundary & Upper Boundary \\
DF-PF & -13.271 & $<0.001^{*}$ & -18.027 & -8.516 \\
DF-EV & 11.634 & $<0.001^{*}$ & 9.853 & 13.414 \\
PF-INV & 15.092 & $<0.001^{*}$ & 10.404 & 19.781 \\
PF-EV & 24.905 & $<0.001^{*}$ & 20.193 & 29.618 \\
INV-EV & 9.813 & $<0.001^{*}$ & 8.845 & 10.782 \\
\hline
\end{tabular}

Key: ROM=Range of Motion; DF=Dorsiflexion; $\mathrm{PF}=$ Plantarflexion; INV=Inversion; EV=Eversion;

$\mathrm{X}=$ Mean Differences; $\mathrm{P}=$ significance; $*$ =Significant

Table D8. Percent Change Scores for ROM.

\begin{tabular}{lccc}
\hline Dependent Variable & Control/DBT Group & GISTM-S/DBT Group & GISTM/DBT Group \\
\hline ROM & & & $26.2^{*}$ \\
DF & 21.2 & 15.5 & $26.3^{*}$ \\
PF & 4.5 & 17.3 & $35.2 *$ \\
INV & 3.9 & 3.3 & 31.3 \\
EV & 39.2 & $44.9 *$ & \\
\hline
\end{tabular}

Key" ROM=Range of Motion; DF=Dorsiflexion; PF=Plantarflexion; INV=Inversion; EV=Eversion;

GISTM-S=Graston Instrumented-Assisted Soft Tissue Mobilization; *=Most percent change by group. 
Table D9. Repeated Measures ANOVA Results for SEBT.

\begin{tabular}{lccccc}
\hline \multicolumn{1}{c}{ Dependent Variable } & df & F & P & ES & $1-\beta$ \\
\hline SEBT & & & & & \\
Time & 1 & 58.493 & $<0.001^{*}$ & 0.639 & 1.000 \\
Group & 2 & 0.291 & 0.749 & 0.017 & 0.024 \\
Time x Group & 2 & 1.992 & 0.152 & 0.108 & 0.169 \\
Direction & 2 & 71.355 & $<0.001^{*}$ & 0.684 & 1.000 \\
Direction x Group & 4 & 0.412 & 0.800 & 0.024 & 0.042 \\
Time x Direction & 2 & 0.504 & 0.606 & 0.015 & 0.038 \\
Time x Direction x Group & 4 & 1.942 & 0.159 & 0.105 & 0.163 \\
\hline
\end{tabular}

Key: SEBT=Star Excursion Balance Test; $\mathrm{df}=$ degrees of freedom; $\mathrm{P}=$ significance; $\mathrm{ES}=$ effect size; 1$\beta=$ power; $*=$ Significant

Table D10. Paired Samples t-Test Results for Pre-test and Post-test for SEBT (Mean Differences).

\begin{tabular}{|c|c|c|c|c|c|}
\hline \multirow[t]{2}{*}{ Dependent Variable } & \multirow[t]{2}{*}{ X } & \multicolumn{2}{|c|}{$98 \%$ CI } & \multirow[t]{2}{*}{$\mathrm{t}$} & \multirow[t]{2}{*}{$\mathrm{P}$} \\
\hline & & Lower & Upper & & \\
\hline \multicolumn{6}{|l|}{ SEBT } \\
\hline A & -8.011 & -11.568 & -4.454 & -5.490 & $<0.001 *$ \\
\hline PM & -6.625 & -9.425 & -3.825 & -5.769 & $<0.001 *$ \\
\hline PL & -7.552 & -10.505 & -4.601 & -6.237 & $<0.001 *$ \\
\hline
\end{tabular}

Key: SEBT=Star Excursion Balance Test; A=Anterior; PM=Posteromedial; PL=Posterolateral; $\mathrm{t}=\mathrm{T}$-test; $\mathrm{P}=$ significance $*$ =Significant

Table D11. Pairwise Comparisons for SEBT (Mean Differences).

\begin{tabular}{lcccc}
\hline \multicolumn{2}{c}{ Dependent Variable } & $\mathrm{X}$ & $\mathrm{P}$ & \multicolumn{2}{c}{$99 \%$ Confidence Interval } \\
\hline & & & Lower Boundary & Upper Boundary \\
A-PM & 4.562 & $0.001^{*}$ & 1.146 & 7.978 \\
A-PL & 17.339 & $0.001^{*}$ & 12.481 & 22.196 \\
PM-PL & 12.777 & $<0.001^{*}$ & 7.055 & 18.499 \\
\hline
\end{tabular}

Key: SEBT=Star Excursion Balance Test; A=Anterior; PM=Posteromedial; PL=Posterolateral; X=Mean

Differences; $\mathrm{P}=$ significance; $*=$ Significant

Table D12. Percent Change Scores for the SEBT.

\begin{tabular}{lccc}
\hline Dependent Variable & Control/DBT Group & GISTM-S/DBT Group & GISTM/DBT Group \\
\hline SEBT & & & $18.2^{*}$ \\
A & 8.1 & 7.7 & $15.6^{*}$ \\
PM & 8.2 & 5.7 & 13.3 \\
PL & 13.5 & $15.8^{*}$ & 13.3 \\
\hline
\end{tabular}

Key: SEBT=Star Excursion Balance Test; A=Anterior; PM= Posteromedial; PL=Posterolateral; GISTM$\mathrm{S}=$ Graston Instrumented-Assisted Soft Tissue Mobilization-Sham; *=Most percent change by group. 
Figure D1. Descriptive Statistics of Pre-Test and Post-Test for FAAM.

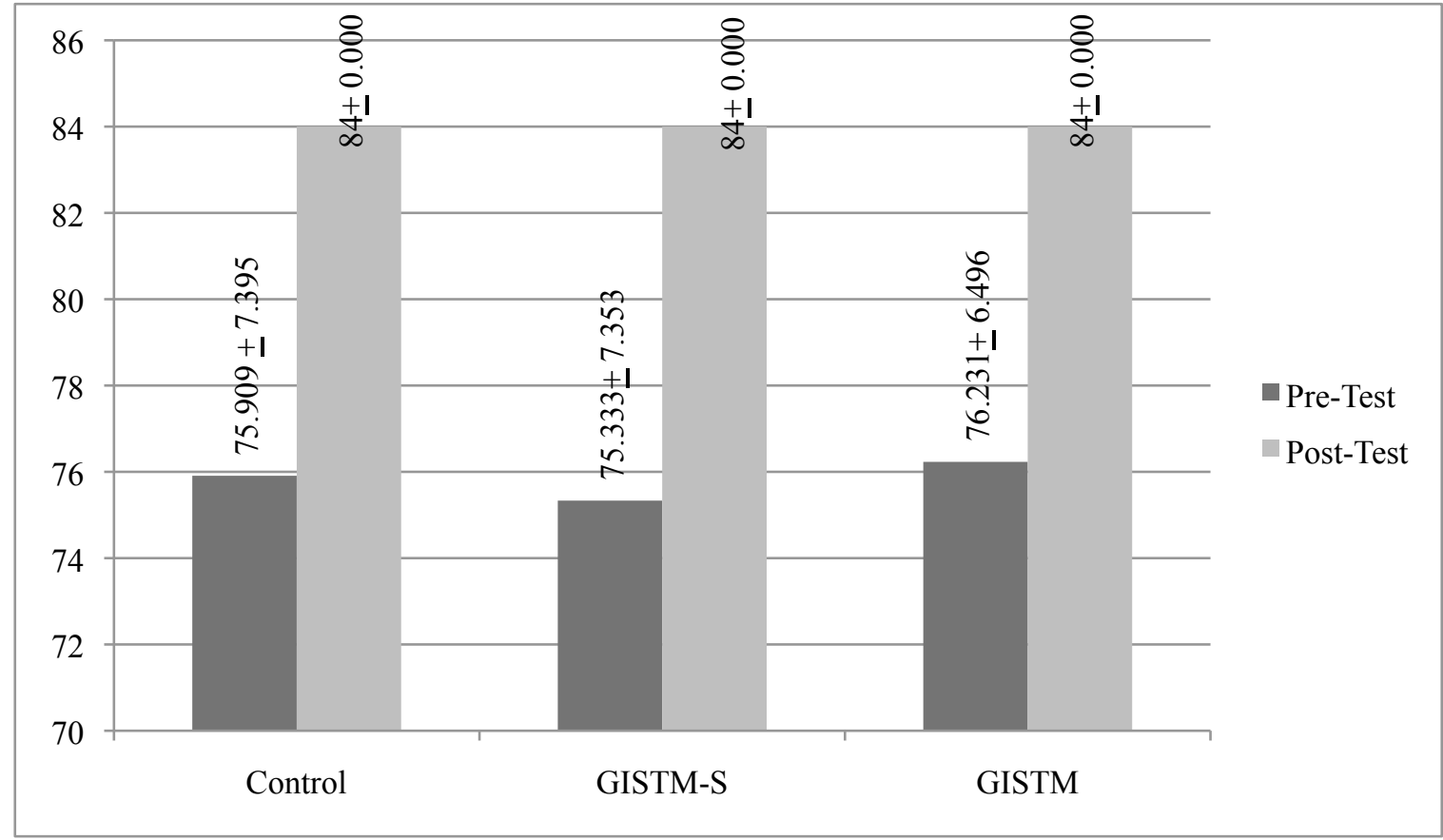

Key: FAAM=Foot and Ankle Ability Measures; GISTM-S=Graston InstrumentedAssisted Soft Tissue Mobilization-Sham; GISTM=Graston Instrumented-Assisted Soft Tissue Mobilization.

Figure D2. Descriptive Statistics of Pre-Test and Post-Test for FAAM Sport.

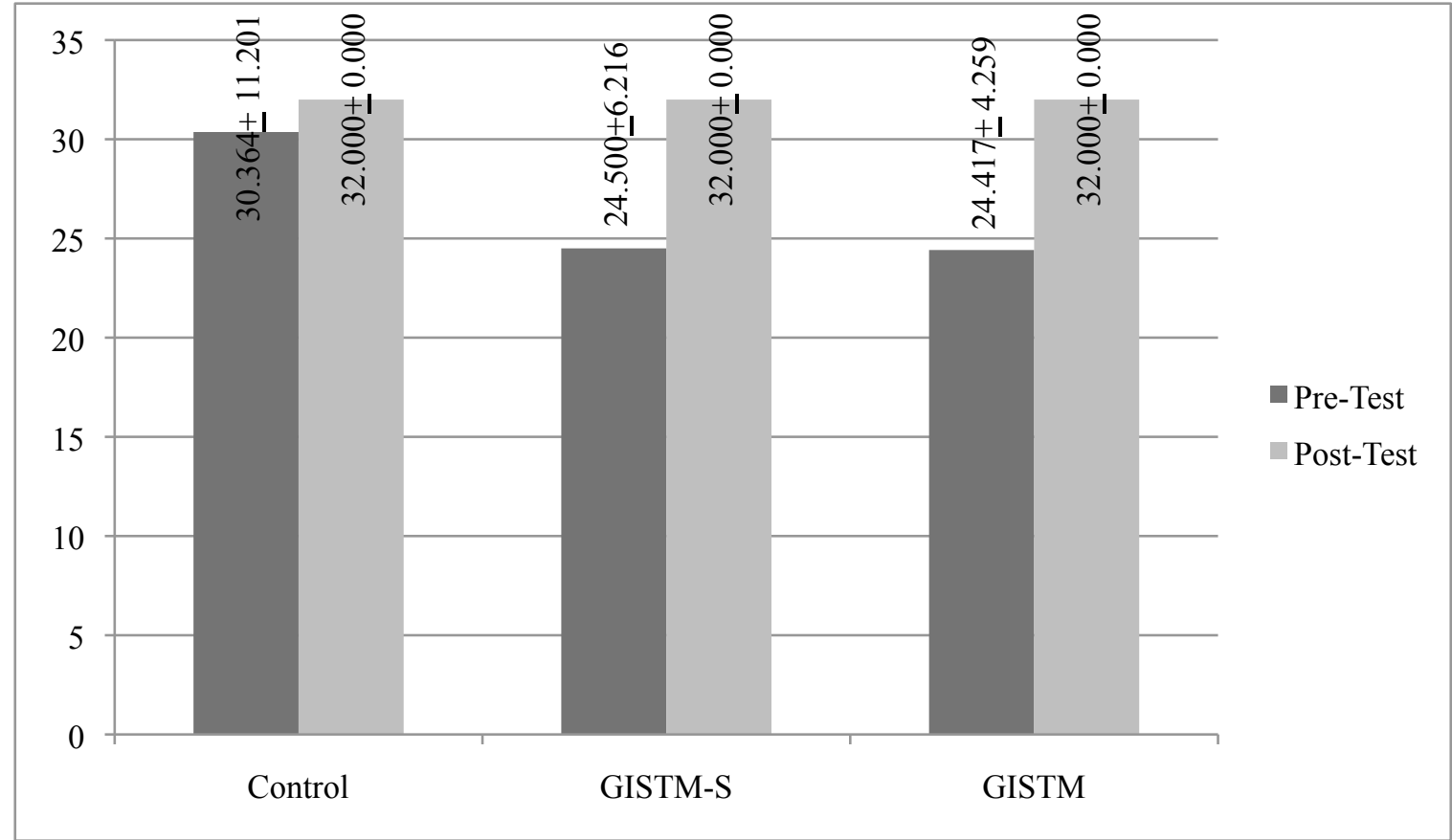

Key: FAAM Sport=Foot and Ankle Ability Measures Sport; GISTM-S=Graston Instrumented-Assisted Soft Tissue Mobilization-Sham; GISTM=Graston InstrumentedAssisted Soft Tissue Mobilization. 
Figure D3. Descriptive Statistics of Pre-Test and Post-Test for VAS.

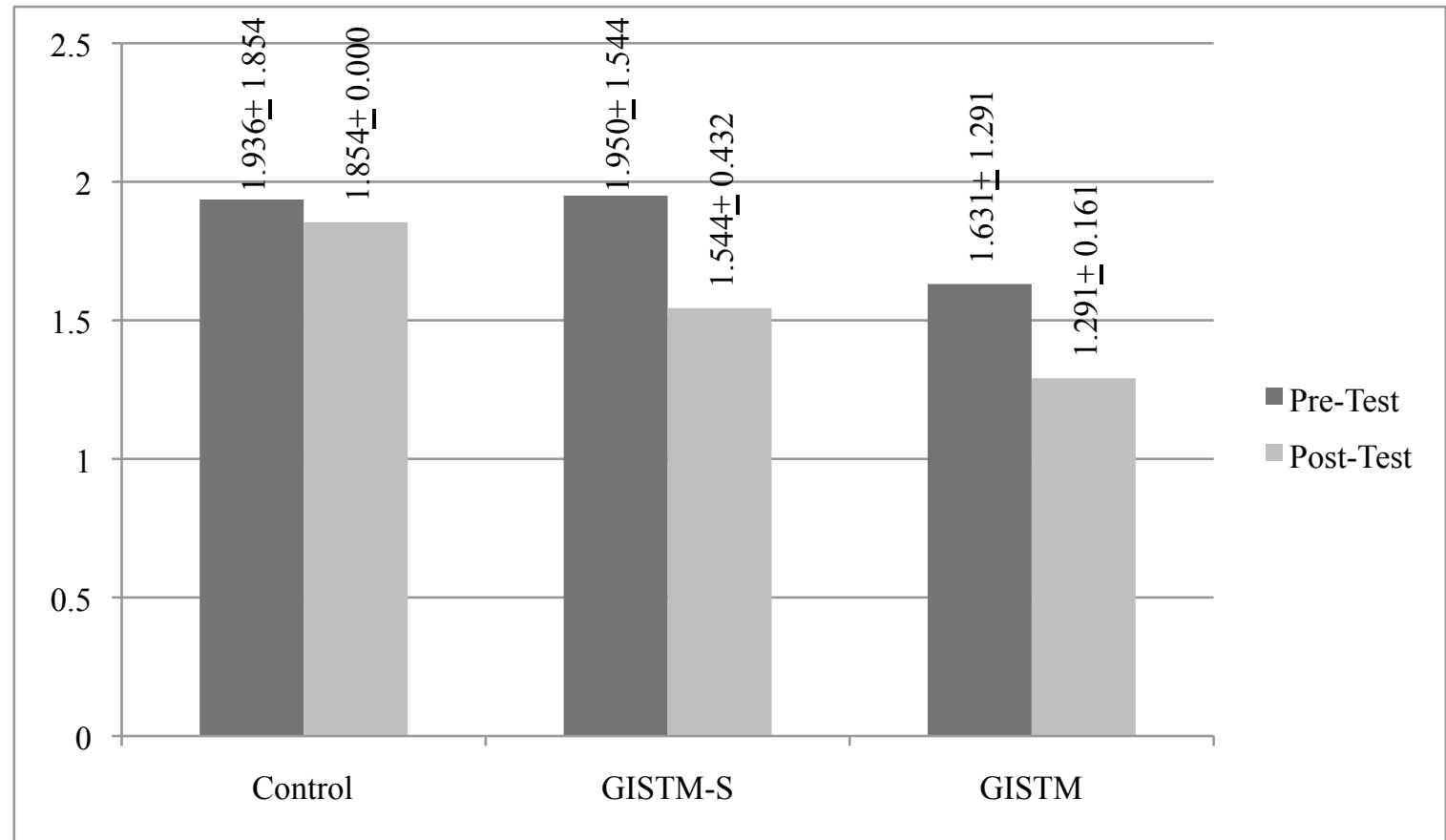

Key: VAS=Visual Analog Scale; GISTM-S=Graston Instrumented-Assisted Soft Tissue Mobilization-Sham; GISTM=Graston Instrumented-Assisted Soft Tissue Mobilization.

Figure D4. Descriptive Statistics of Pre-Test and Post-Test for ROM.

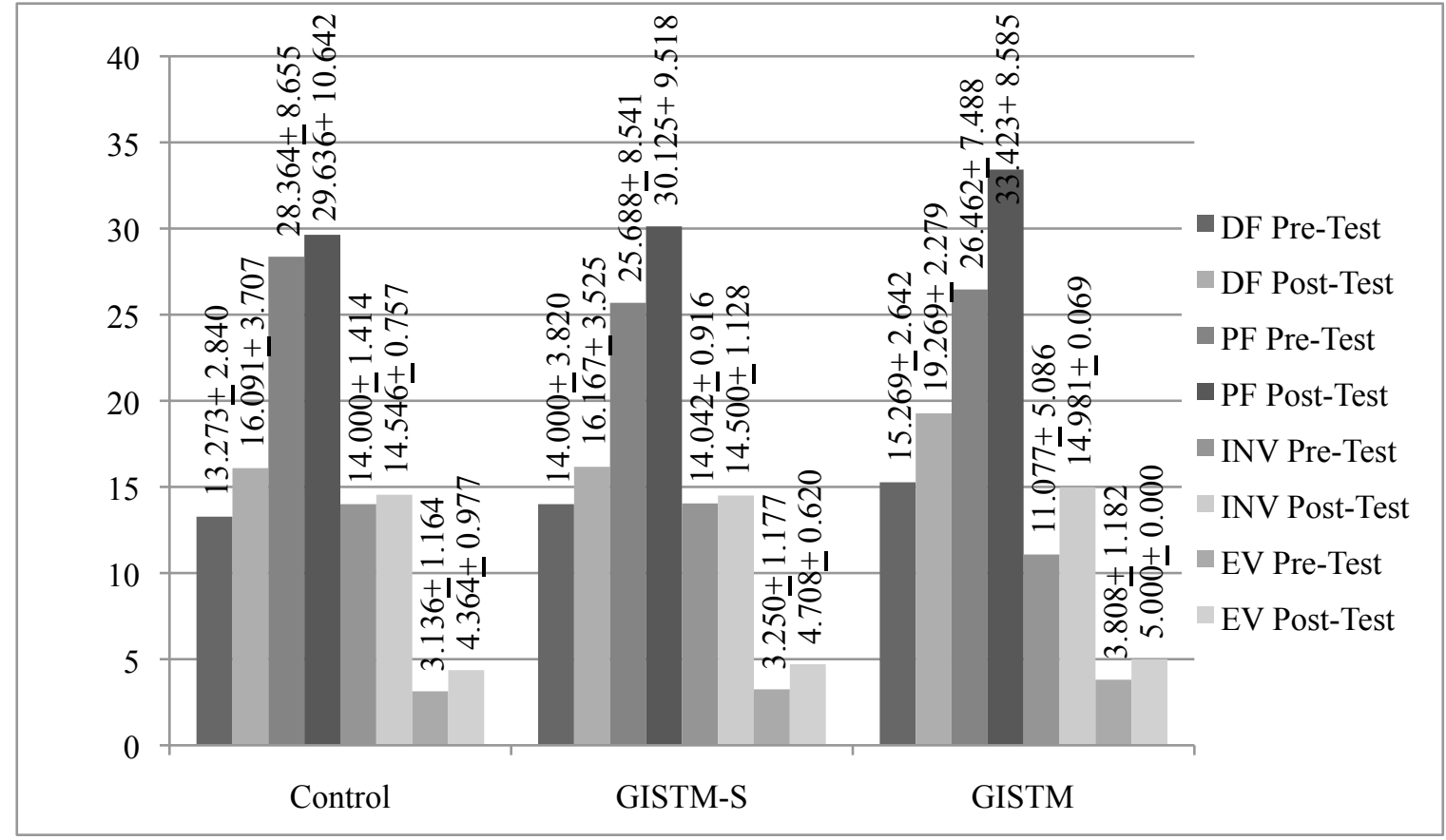

Key: ROM=Range of Motion; GISTM-S=Graston Instrumented-Assisted Soft Tissue Mobilization-Sham; GISTM=Graston Instrumented-Assisted Soft Tissue Mobilization; $\mathrm{DF}=$ Dorsiflexion; $\mathrm{PF}=$ Plantarflexion; $\mathrm{INV}=$ Inversion; $\mathrm{EV}=$ Eversion 
Figure D5. Descriptive Statistics of Pre-Test and Post-Test for SEBT.

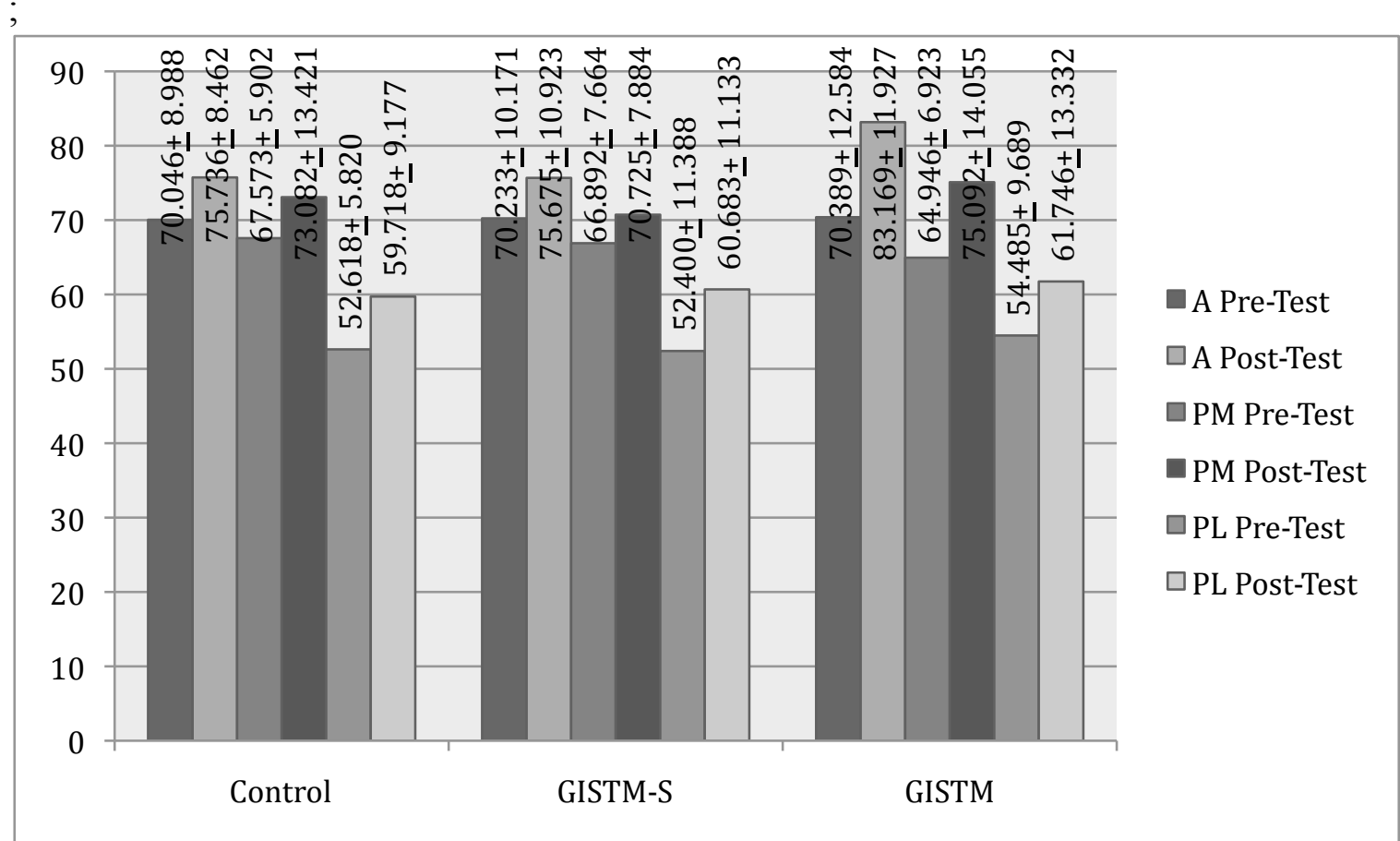

Key: SEBT=Star Excursion Balance Test; GISTM-S=Graston Instrumented-Assisted Soft Tissue Mobilization-Sham; GISTM=Graston Instrumented-Assisted Soft Tissue Mobilization; $\mathrm{A}=$ Anterior; $\mathrm{PM}=$ Posteromedial; $\mathrm{PL}=$ Posterolateral

Figure D6. Ultrasonography of Normal ATF Ligament. ${ }^{98}$

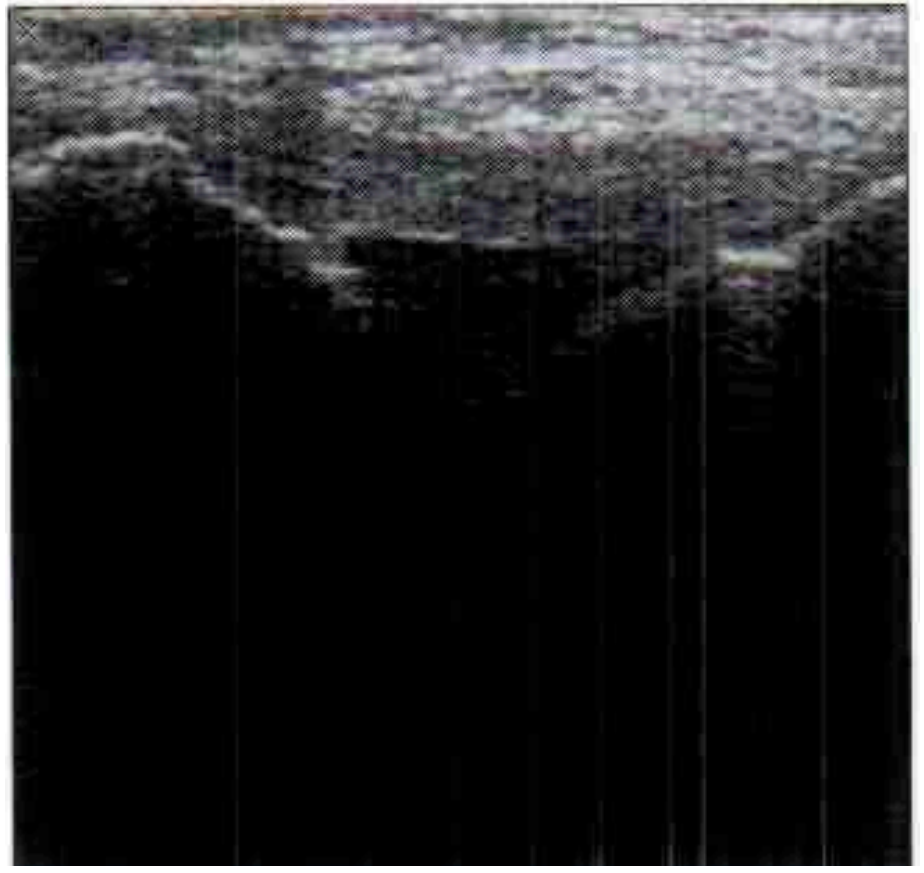


Figure D7. Ultrasonography of Normal CF Ligament. ${ }^{98}$

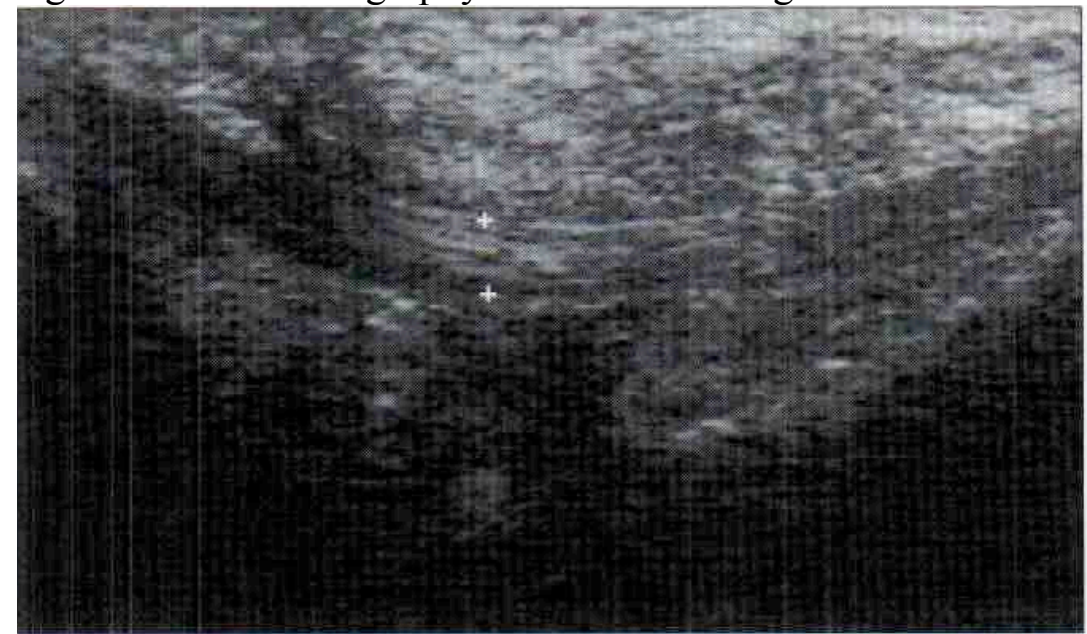

Figure D8. GISTM Group Pre-Test Ultrasonography of ATF Ligament.

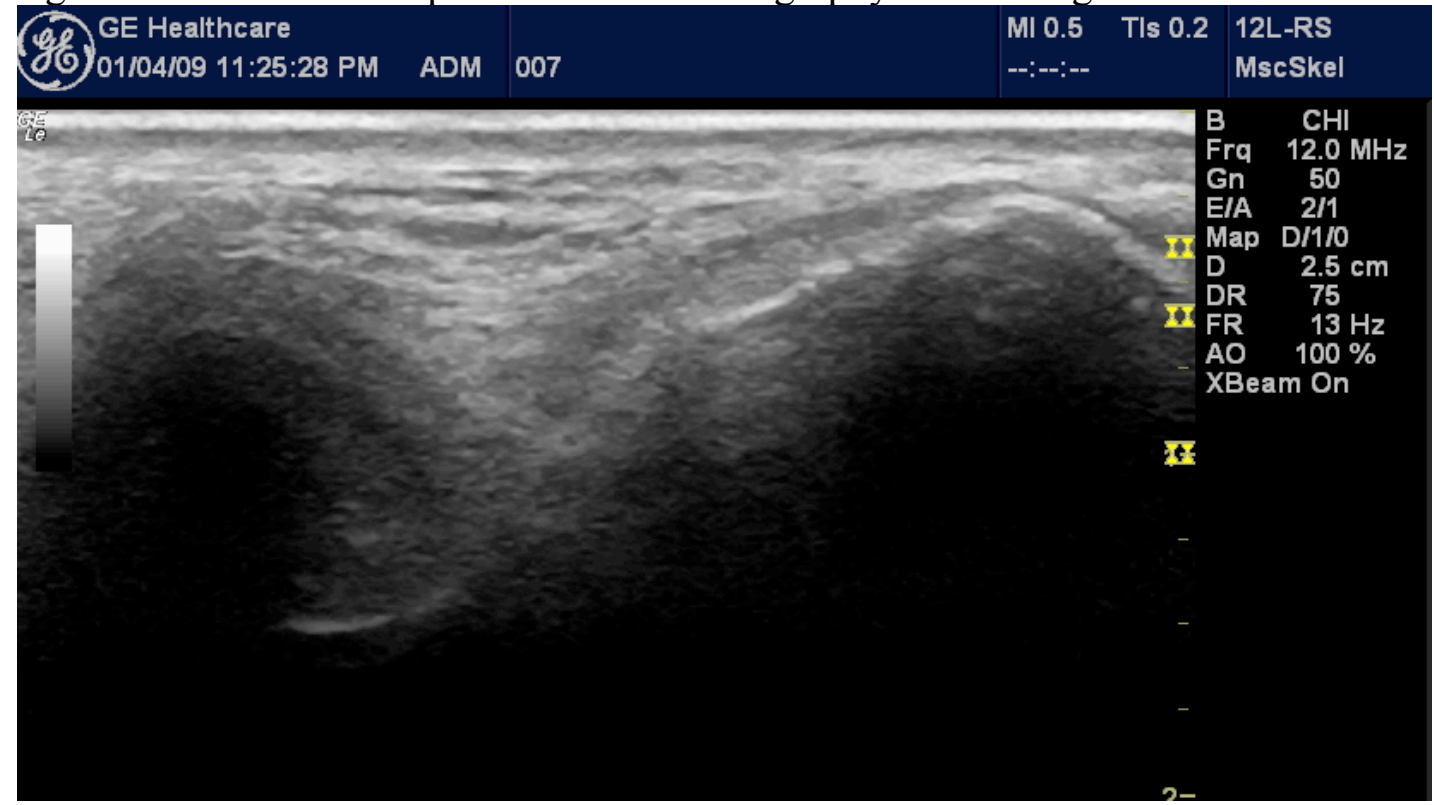


Figure D9. GISTM Group Post-Test Ultrasonography of ATF Ligament.

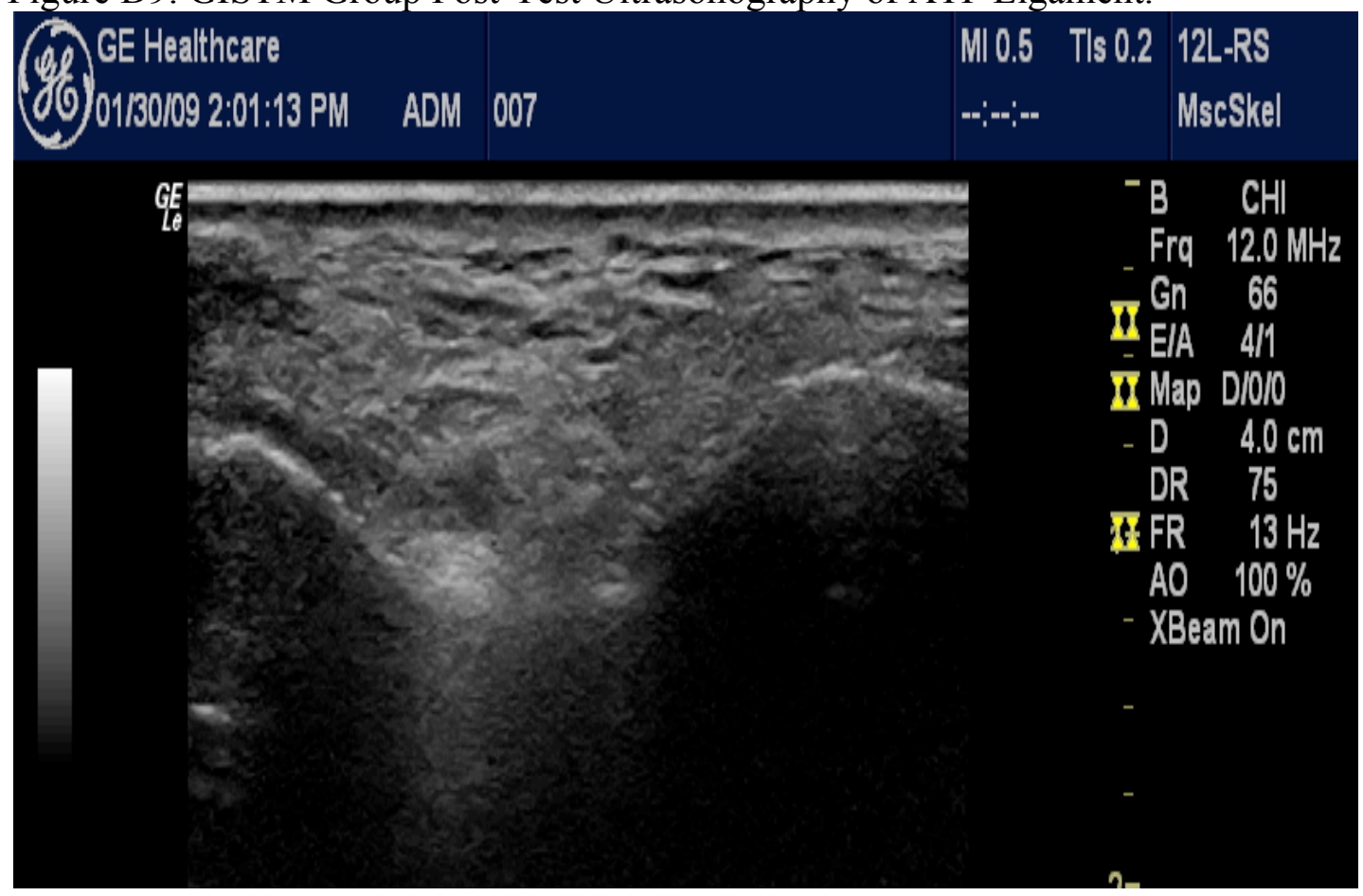

Figure D10. GISTM Group Pre-Test Ultrasonography of CF Ligament.

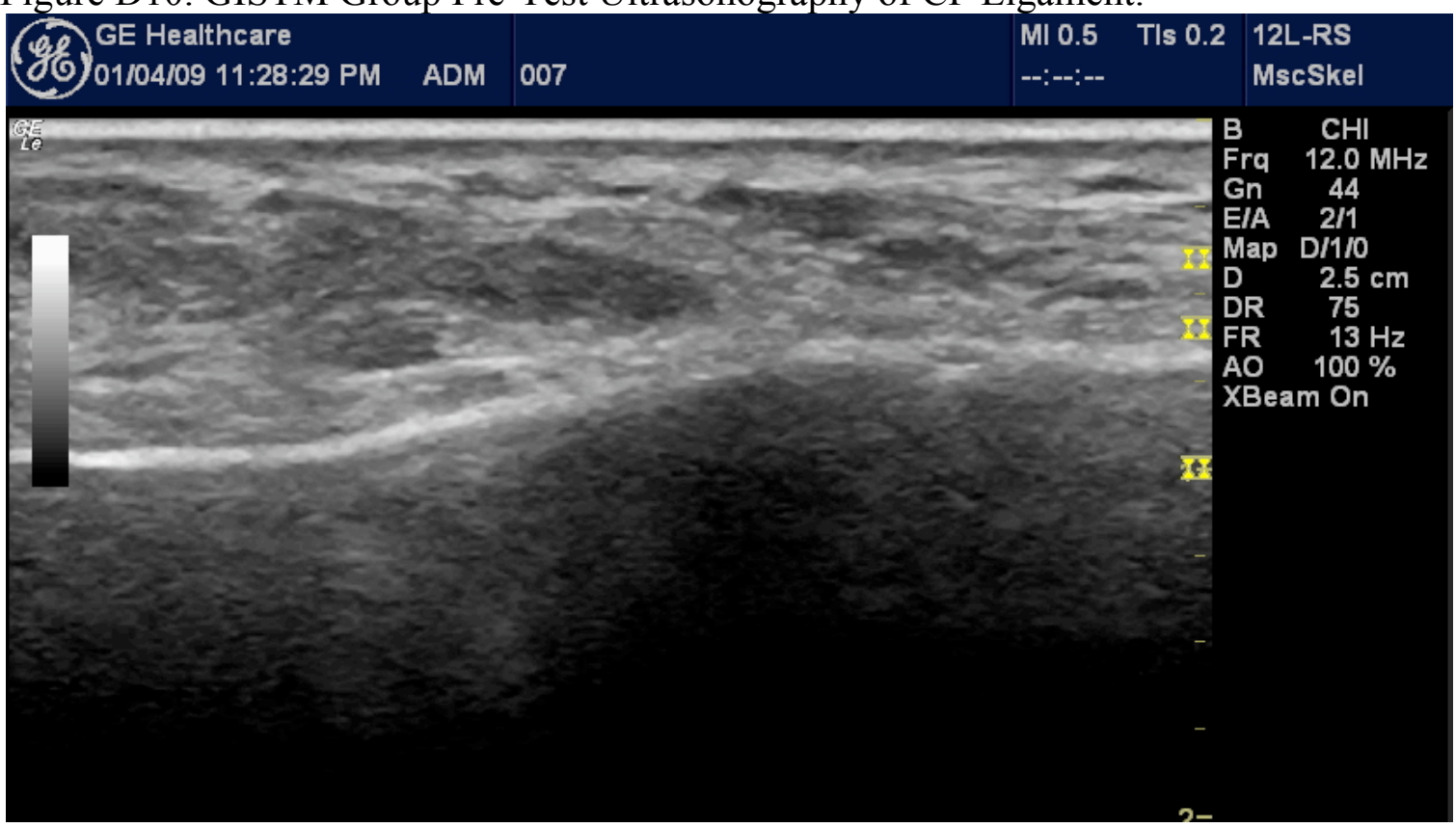


Figure D11. GISTM Group Post-Test Ultrasonography of CF Ligament.

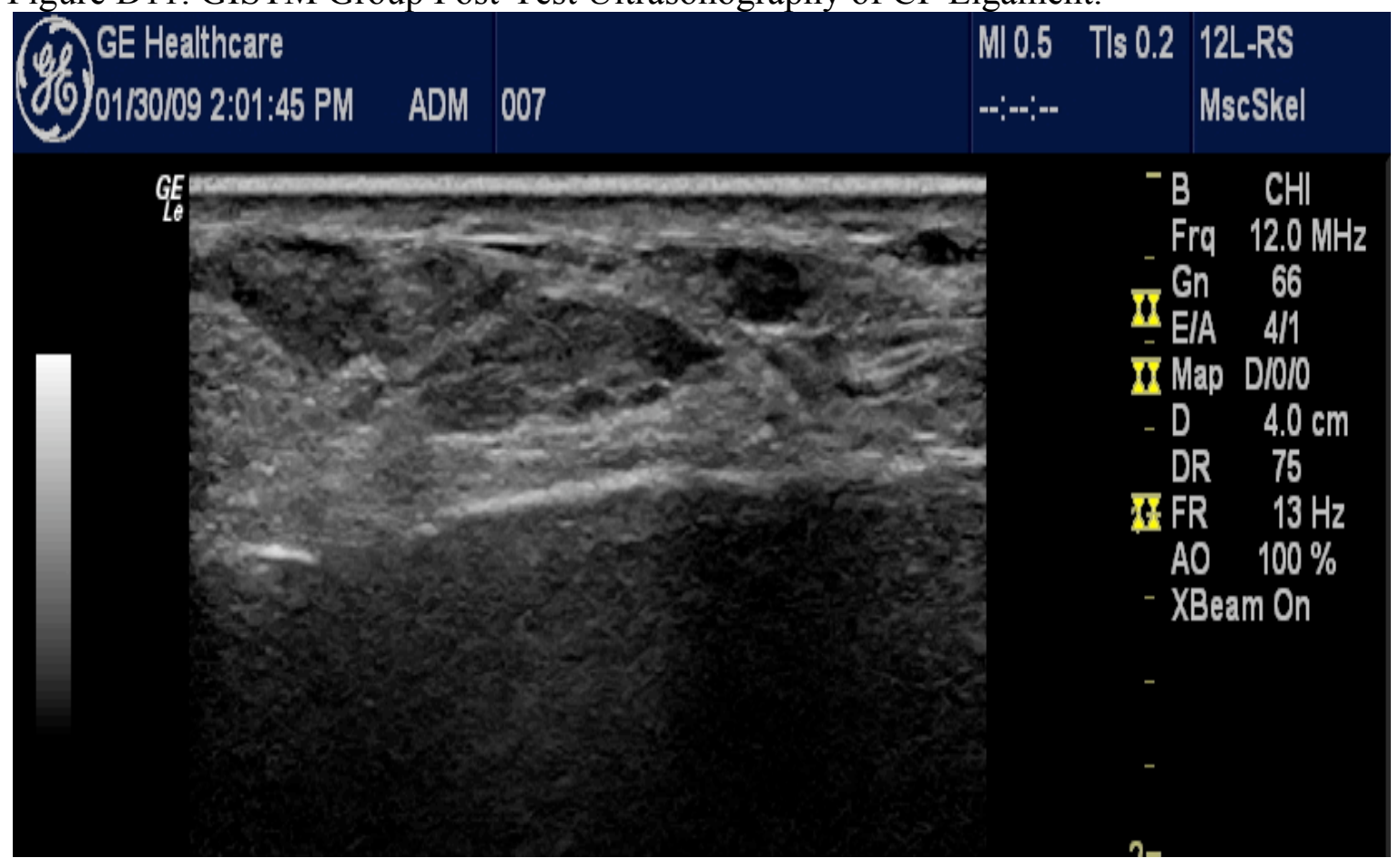

Figure D12. GISTM-Sham Group Pre-Test Ultrasonography of ATF Ligament.

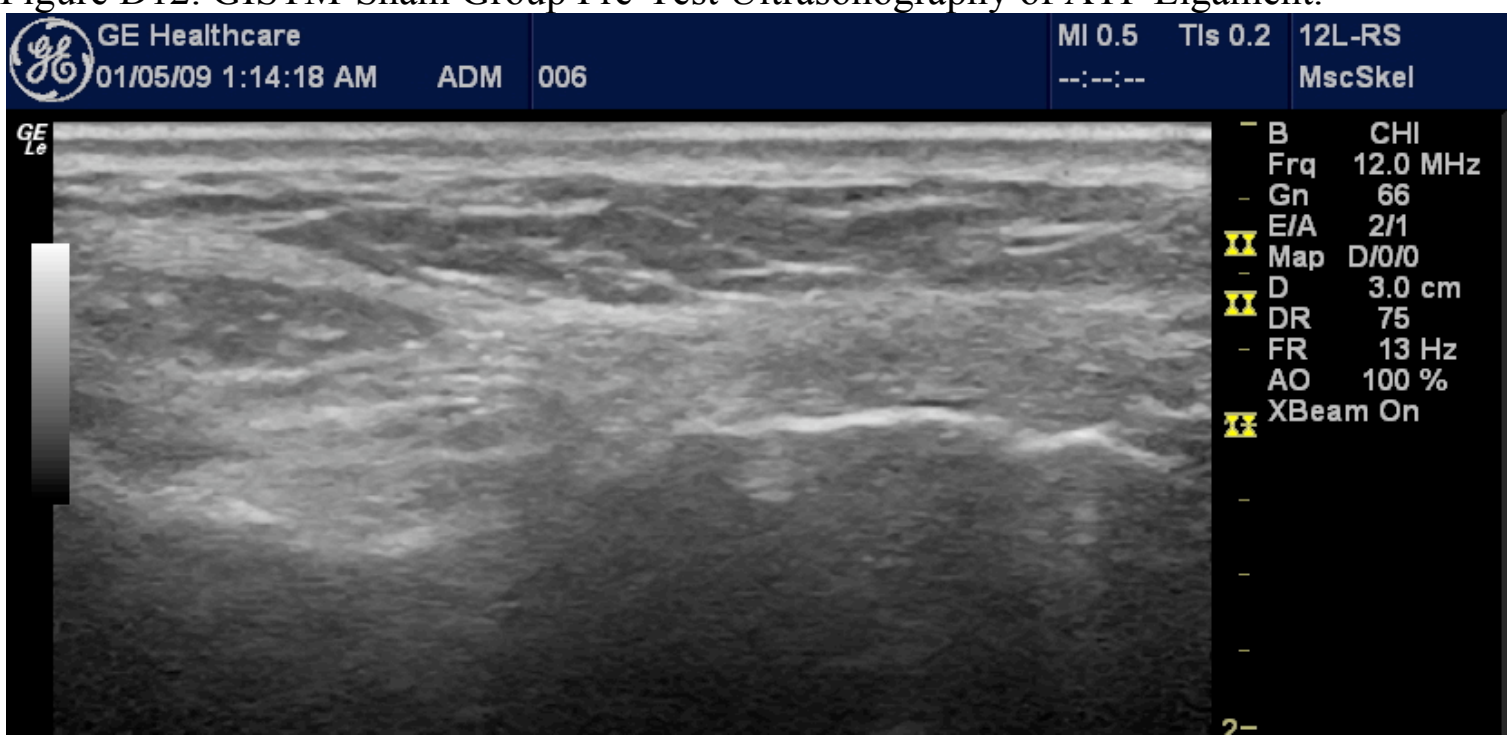


Figure D13. GISTM-Sham Group Post-Test Ultrasonography of ATF Ligament.

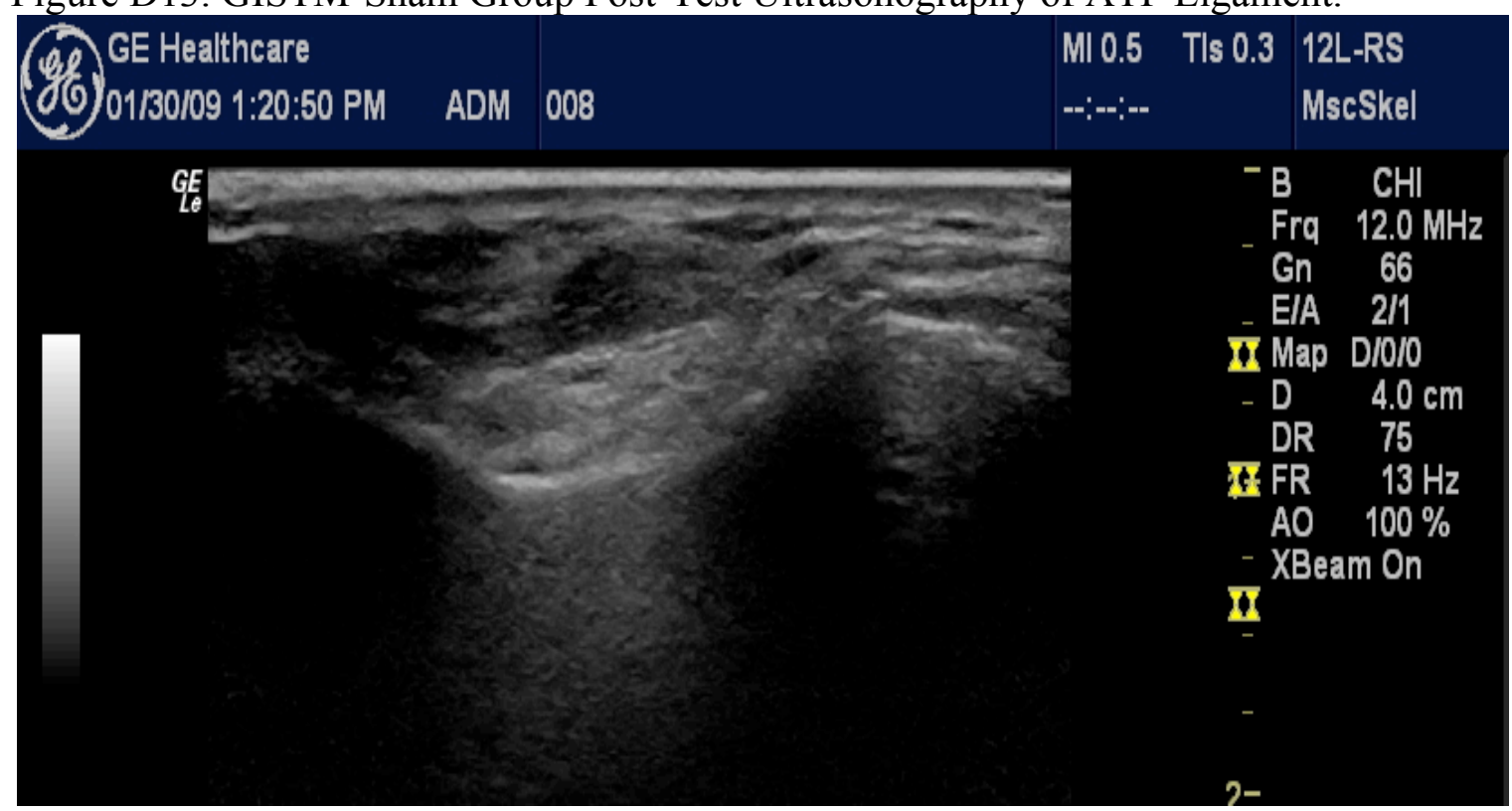

Figure D14. GISTM-Sham Group Pre-Test Ultrasonography of CF Ligament.

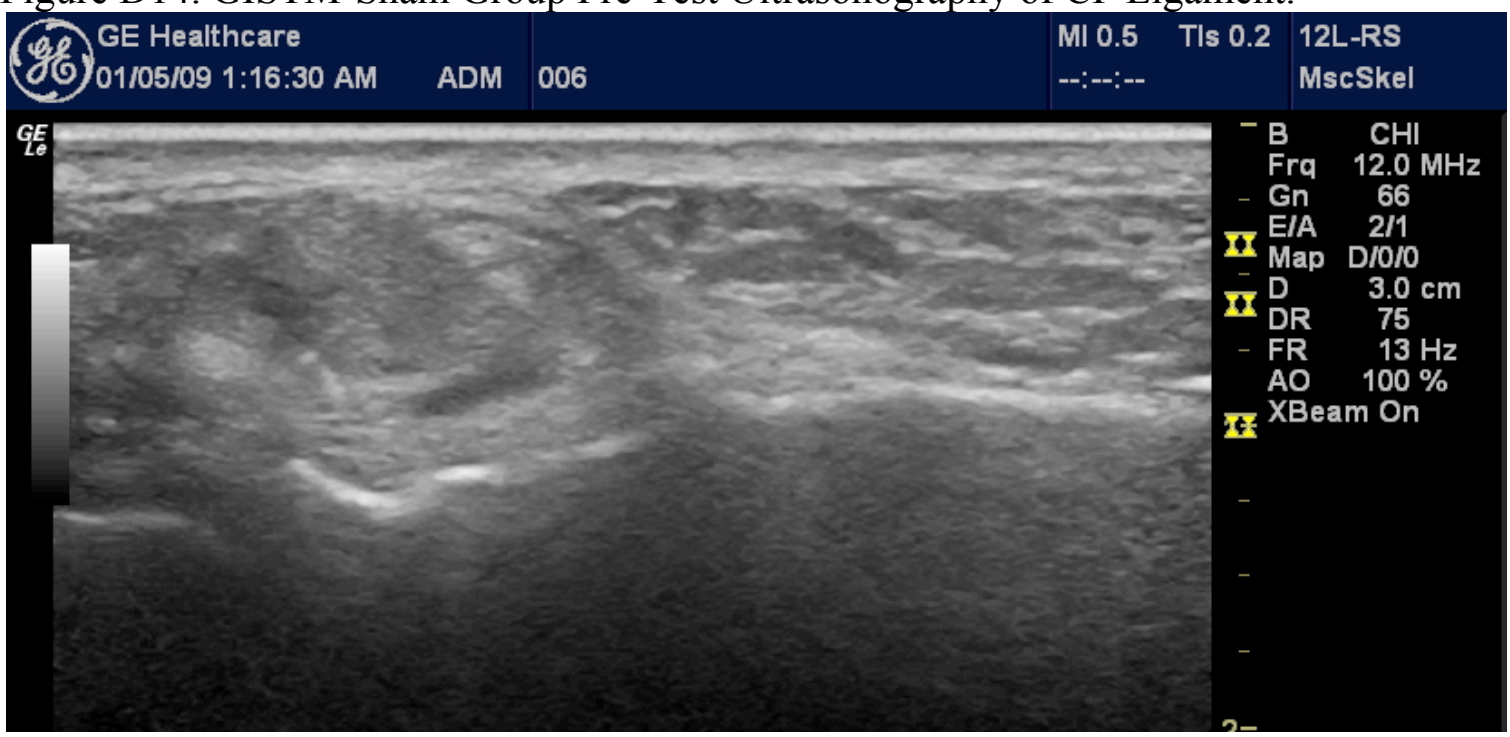


Figure D15. GISTM-Sham Group Post-Test Ultrasonography of CF Ligament.

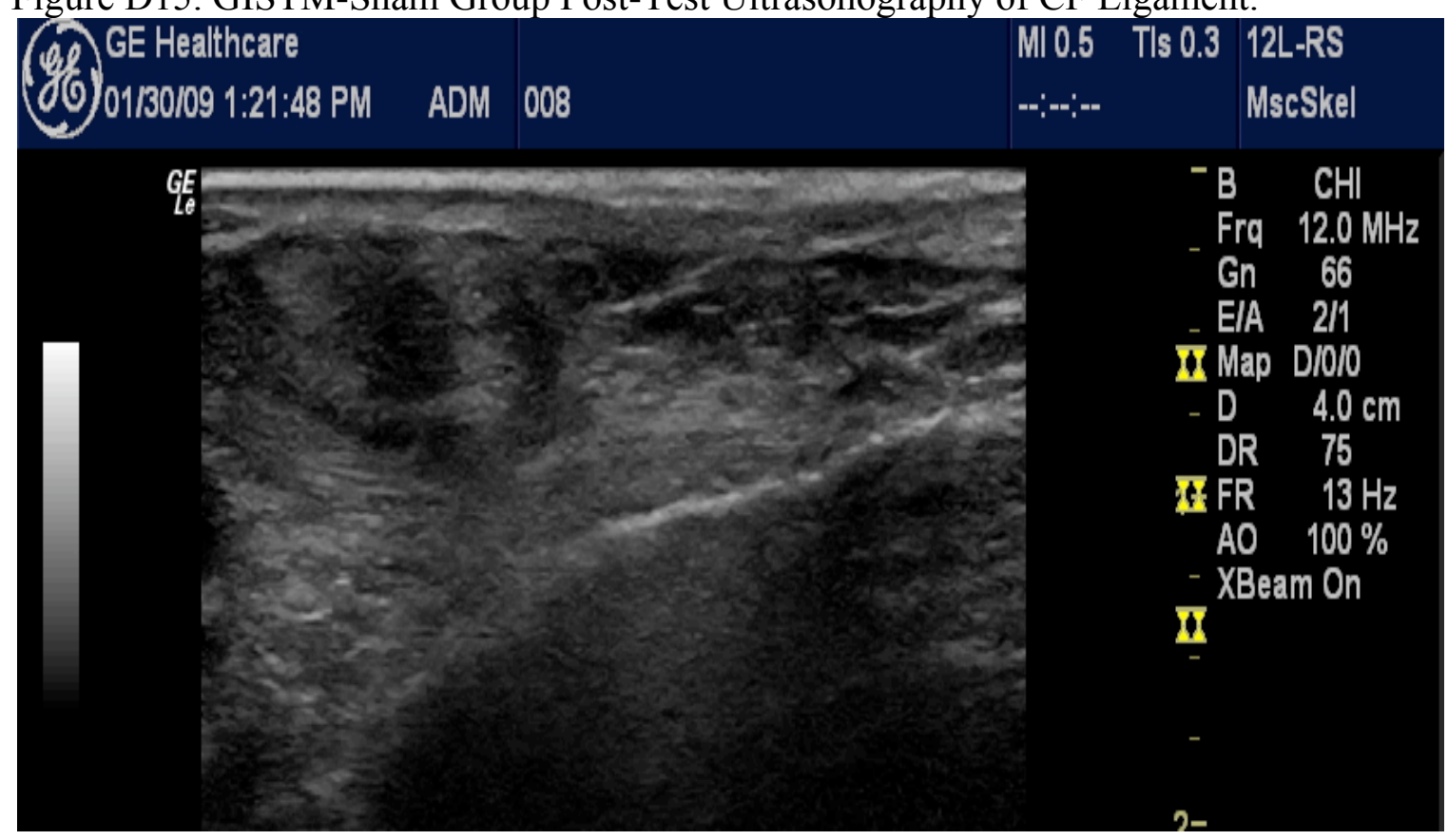




\section{APPENDIX E \\ RECOMMEDATIONS FOR FUTURE RESEARCH}

1. Conduct the study during the spring or summer when there are fewer activities at the school and school cancelations relative to the weather will not be a problem due to weather.

2. Conduct the study during the subject's preseason or offseason.

3. Increase the number of researchers collecting data to two or four to aid in data entry and treatment.

4. Conduct the study using a board certified ultrasonographer to capture the ultrasound images.

5. Conduct the study using real time ultrasonography images instead of stilled images.

6. Increase the length of the study to six or eight weeks.

7. Conduct the study using a smaller probe size for athletes to accommodate for a smaller foot.

8. Increase the number of subjects per experimental groups to 30 to 45 to increase power and effect size.

9. Conduct the study using recreational, collegiate, high school, and youth athletes.

10. Conduct the study using non-physically active subjects with chronic ankle instability.

11. Conduct the study with evaluating group by grade to compare tissue damage.

12. Conduct the study in a controlled laboratory setting or an undisturbed, restrained area.

13. Conduct a prospective study as a pre-season injury prevention program each year, and then perform a longitudinal study to look at the injury rates for this season, and successive years. 


\section{ADDITIONAL REFERENCES}

84. Brown CN. Mynark R. Balance deficits in recreational athletes with chronic ankle instability. J Athl Train. 2007;42(3):367-373.

85. Benjamin M, Ralphs JR. Fibrocartilage in tendons and ligaments - an adaptation to compressive load. J Anat. 1998;193:481-494.

86. Perry JT, Statler JD. Advances in vascular imaging. Surg Clin North Am. 2007;87(5):975-93, vii.

87. Zoch C, Fialka-Moser V, Quittan M. Rehabilitation of ligamentous ankle injuries: a review of recent studies. Br J Sports Med. 2003;37:291-295.

88. Beynnon BD, Renstrom PA, Haugh L, Uh BS, Barker H. A prospective, randomized clinical investigation of the treatment of first-time ankle sprains. Am J Sports Med. 2006;34:1401-12.

89. Pollard H, Sim P, McHardy A. Lateral ankle injury: literature review and report of two cases. Australas Chiropr Osteopathy. 2002;10(1):21-30.

90. Boyce SH, Quigley MA, Campbell S. Management of ankle sprains: a randomized controlled trial of the treatment of inversion injuries using elastic support bandage or an aircast ankle brace. Br J Sports Med. 2005;39:91-96.

91. Moore KL, Dalley AF. Clinically Oriented Anatomy. 4th ed. Baltimore, MD: Lippincott, Williams, \& Wilkins; 1999: 551-555,577,583,588,589.

92. Levangie PK, Norkin CC. Joint Structure and Function: A Comprehensive Analysis. 3ed. Philadelphia, PA.: F.A. Davis; 2001:2,372-376.

93. Nordin M, Frankel VH. Basic Biomechanics of the Musculoskeletal System. 3 ed. Baltimore, MD: Lippincott Williams \& Wilkins; 2001:251.

94. Bosien WR, Staples OS, Russell SW. Residual Disability following acute ankle sprains. J Bone Joint Surg Am. 1955;37:1237-1243.

95. Vela L, Tourville TW, Hertel J. Physical examination of acutely injured ankles: an evidence-based approach. Ath Ther Today. 2003;8(5):13-19.

96. Prentice WE. Rehabilitation techniques for Sports Medicine and Athletic Training. $4^{\text {th }}$ ed. New York, NY: Mc Graw-Hill Companies, Inc; 2004:28-30,40-47,608-648.

97. Prentice WE. Arnheim's Principles of Athletic Training a Competency-Based Approach. $12^{\text {th }}$ ed. New York, NY: Mc Graw-Hill Companies, Inc; 2006:272292,560-597. 
98. Wilson JK, Sevier TL. A review of treatment for carpal tunnel syndrome. Disabil Rehabil. 2003;25(3):113-119.

99. Arnolt M. (n.d). Graston Technique. In Graston Technique: Simple Techonology Improving Injury Treatment and Rehabilitation. Retrieved. 2008 July 7, from Website: http://www.grastontechnique.com.

100. Fessell DP, VanderSchueren GM, Jacobson JA, Ceulemans RY, Prasad A, Craig JG, Bouffard JA, Shirazi KK, Van Holsbeeck MT. US of the ankle: technique, anatomy, and diagnosis of pathologic conditions. RadioGraphics. 1998;18(2):325340 .

101. Peetrons PA, Silvestre A, Cohen M, Creteur V. Ultrasonography of ankle ligaments. Can Assoc Radiol J. 2002;53(1):6-13.

102. Guillodo Y, Guennoc X, Saraux A. Usefulness of ultrasonographic detection of talocrural effusion in ankle sprains. J Ultrasond Med. 2007;26:831-836.

103. Naredo E, Moller I, Moragues C, De Agustin JJ, Scheel AK, Grassi W, De Miguel E, Backhaus M, Balint P, Bruyn GAW, D’Agostino MA, Filippucci E, Lagnocco A, Kane D, Koski JM, Mayordomo L, Schmidt WA, Swen WAA, Szkudlarek M, Terslev L, Torp-Pedersen S, Uson J, Wakefield RJ, Werner C, EULAR Working Group for Musculoskeletal Ultrasound. Interobeserver reliability in musculoskeletal ultrasonography: results from a "teach the teachers" rheumatologist course. Ann Rheum Dis. 2006;65:14-19.

104. Scheel AK, Schmidt WA, Hermann K-GA, Bruyn GA, D’Agostino MA, Grassi W, Lagnocco A, Koski JM, Machold KP, Naredo E, Swen N, Szkudlarek, Wakefield RJ, Ziswiler HR, Pasewaldt D, Werner C, Backhaus M. Interobserver reliability of rheumatologists performing musculoskeletal ultrasonography: results from a EULAR "train the trainers" course. Ann Rheum Dis. 2005;64:1043-1049.

105. Eechaute C, Vaes P, Aerschot LV, Asman S, Duquet W. The clinimetric qualities of patient-assessed instruments for measuring chronic ankle instability: a systematic review. BMC Musculoskeletal Disorders. 2007;8(6):1-11.

106. Menz HB, Tiedemann A, Kwan MMS, Plumb K, Lord SR. Foot pain in community-dwelling older people: an evaluation of the Manchester foot pain and disability index. Rheumatology (Oxford). 2006;45:863-867.

107. Cook CE, Cleland J, Pietrobon R, Garrow AP, Macfarlane GJ. Calibration of an item pool for assessing the disability associated with foot pain: an application of item response theory to the Manchester foot pain and disability index. Physiotherapy. 2007;93:89-95. 
108. Rockar PA. The subtalar joint: anatomy and joint motion. J Orthop Sports Phys Ther. 1995;21(6):361-372.

109. Dahmer G. Biomechanics. ATTR 627. Class notes. West Virginia University. School of Physical Education. January 2008.

110. Norkin CC, White J. Measurement of Joint Motion. 2ed. Phildelphia, PA: F.A. Davis; 1995:150.

111. Ferris CM, Freedman AD. Neuromuscular and biomechanical lower extremity training for female athletes. Athc Ther Today. 2001;6(4):54-63.

112. Garrsion JG, Hart JM, Palmieri RM, Kerrigan DC, Ingersoll CD. Lower extremity EMG in male and female college soccer players during single-leg landing. J Sport Rehabil. 2005;14:48-57.

113. Forkin DM, Koczur C, Battle R, Newton RA. Evaluation of kinesthetic deficits indicative of balance control in gymnasts with unilateral chronic ankle sprains. $J$ Orthop Sports Phys Ther. 1996;23(4):245-250.

114. Monaghan K, Delahunt E, Caulfield B. Ankle function during gait in patients with chronic ankle instability compared to controls. Clin Biomech (Bristol, Avon). 2006;21(2):168-74.

115. Beckman SM, Buchanan TS. Ankle inversion injury and hypermobility: effect on hip and ankle muscle electromyography onset latency. Arch Phys Med Rehabil. 1995;76(12):1138-43.

116. Hootman JM, Dick R, Agel J. Epidemiology of collegiate injuries for 15 sports: summary and recommendations for injury prevention initiatives. $J$ Athl Train. 2007;42(2):311-9.

117. Fong DT, Hong Y, Chan LK, Yung PS, Chan KM. A systematic review on ankle injury and ankle sprain in sports. Sports Med. 2007;37(1):73-94.

118. Ross SE, Guskiewicz KM, Gross MT. Assessment tools for identifying functional limitations associated with functional ankle instability. J Athl Train. 2008;43(1):4450 .

119. Beynnon BD, Murphy DF, Alsoa DM. Predictive factors for the lateral ankle sprains: a literature review. J Athl Train. 2002;37(4):376-380.

120. Beynnon BD, Vacek PM, Murphy D, Alosa D, Paller D. First-time inversion ankle ligament trauma: the effects of sex, level of competition, and sport on the incidence of injury. Am J Sports Med. 2005;33:1485-1491. 
121. Bahr R, Pena F, Shine J, Lew WD, Lindquist C, Tyrdal S, Engebretsen L. Mechanics of the anterior drawer and talar tilt tests. A cadaveric study of lateral ligament injuries of the ankle. Acta Orthop Scand. 1997;68(5):435-41.

122. Gaebler C, Kukla C, Brietenseher MJ, Nellas ZJ, Mittlboeck M, Trattnig S, Vecsei V. Diagnosis of lateral ankle ligament injuries. Comparison between talar tilt, MRI and operative findings in 112 athletes. Acta Orthop Scand. 1997;68(3):286-90.

123. Lahde S, Putkonen M, Puranen J, Raatikainen T. Examination of the sprained ankle: anterior drawer test or arthrography? Eur J Radiol. 1988;8(4):255-7.

124. Stiell IG, McKnight RD, Greenberg GH et al. Implementation of the Ottawa ankle rules. JAMA 1994:271:827-832.

125. Fujii T, Luo ZP, Kitaoka HB, An KN.The manual stress test may not be sufficient to differentiate ankle ligament injuries. Clin Biomech (Bristol, Avon). 2000;15(8):619-23.

126. Hertel J, Denegar CR, Monroe MM, Stokes WL. Talocrural and subtalar joint instability after lateral ankle sprain. Med Sci Sports Exerc. 1999;31(11):1501-1508.

127. Denegar CR, Hertel J, Fonseca J. The effect of lateral ankle sprain on dorsiflexion range of motion, posterior talar glide, and joint laxity. J Orthop Sports Phys Ther. 2002;32(4):166-173.

128. Kovaleski JE, Norrell PM, Heitman RJ, Hollis JM, Pearsall AW. Knee and ankle position, anterior drawer laxity, and stiffness of the ankle complex. J Athl Train. 2008;43(3):242-8.

129. Kovaleski JE, Hollis J, Heitman RJ, Gurchiek LR, Pearsall AW $4^{\text {th }}$. Assessment of ankle-subtalar-joint-complex laxity using an instrumented ankle arthrometer: an experimental cadaveric investigation. J Athl Train. 2002;37(4):467-474.

130. Hertel J, Denegar CR. A rehabilitation paradigm for restoring neuromuscular control following athletic injury. Athl Ther Today. 1998;3(5):12-16.

131. Bleakley CM, McDonough SM, MacAuley DC. Cryotherapy for acute ankle sprains: a randomized controlled study of two different icing protocols. $\mathrm{Br} J$ Sports Med. 2006;40:700-705.

132. Enwemeka CS, Allen C, Avila P, Bina J, Konrade J, Munns S. Soft tissue thermodynamics before, during, and after cold pack therapy. Med Sci Sport Exerc. 2002;34(1):45-50. 
133. Bleakley C, McDonough S, MacAuley D. The use of ice in the treatment of acute soft-tissue injury: a systematic review of randomized controlled trials. Am J Sports Med. 2004;32:251-261.

134. Tsang KKW, Hertel J, Denegar CR. Volume decreases after elevation and intermittent compression of postacute ankle sprains are negated by gravity-dependent positioning. J Athl Train. 2003;38(4):320-324.

135. Kovaleski JE, Kovaleski SJ, Pearsall AW IV. Functional rehabilitation after lateral ankle injury. ATT. 2006;11(3):52-55.

136. Mattacola CG, Dwyer MK. Rehabilitation of the ankle after acute sprain or chronic instability. $J$ Athl Train. 2002;37(4):413-429.

137. Thorogood L. Proprioception exercises following ankle sprain. Emerg Nurse. 2003;11(8):33-36.

138. Docherty CL, Arnold BL, Gansneder BM, Hurwitz S, Gieck J. Functionalperformance deficits in volunteers with functional ankle instability. $J$ Athl Train. 2005;40(1):30-34. 